\title{
Nature et importance des pertes économiques dans l'utilisation de l'informatique en Europe en 1988
}

\section{Introduction}

Nous n'avons plus à être pour ou contre l'utilisation de l'informatıque dans notre société : aujourd'hui, nous ne pouvons plus que faire en sorte que cette utilisation soit un nouveau progrès dans la société moderne et non une raison supplémentaire de bouleversement de celle-ci.

Les premiers ordinateurs, autres que militaires, sont apparus voici à peine plus de vingt années aux Etat-Unis, puis quelques années plus tard en Europe; actuellement, leur pénétration et l'impact de leur utilisation sont tels qu'il n'est pratiquement aucun secteur économique, aucune parcelle de notre existence personnelle, qui n'en soit plus ou moins affecté.

Et il est maintenant certain que, quelle qu'en soit la forme, notre vie sera encore plus dépendante de l'informatique dans les années qui viennent. Car si, jusqu'à présent, l'utilisation des ordinateurs a été justifiée par la recherche de l'amélioration de la rentabilité (encore qu'il faille bien avouer que, dans de nombreux cas, un certain... snobisme... de l'informatique a motivé son utilisation) il faut bien se rendre compte que, plus on recherche à sophistiquer les systèmes d'information de toute nature, plus l'emploi de l'ordinateur devient indispensable.

Cette nécessité ne doit cependant pas cacher les dangers inhérents à ces systèmes automatiques et/ou automatisés. Le système informatique n'est en fait qu'un ensemble d'équipements divers (de saisie, de traitement, de restitution et de transmission des informations) commandés par des programmes conçus par l'homme et utilisant, dans la plupart des cas, des informations fournies par l'homme. Si l'on considère donc la sécurité d'un tel système, on se rend compte que les incidents possibles peuvent survenir :

- au niveau des équipements: pannes, mauvaises utilisations, erreurs de fonctionnement ;

- au niveau des programmes: erreurs de conception;

- au niveau des utilisateurs : mauvaises informations fournies au système ou mauvaises décisions prises sur les données communiquées par le système, fraudes, sabotages.

La puissance et la complexité des futurs systèmes informatiques deviendront donc tels que les dangers encourus risqueront d'être de plus en plus considérables (jusque et y compris les atteintes à la vie humaine). C'est pour mieux comprendre ce phénomène et pour essayer de préparer les réponses possibles à la société informatique des années 80 que l'Association de Genève a conçu une étude prospective qui dresse un cadre général aux développements possibles de l'informatique dans les douze années à venir et qui cherche à esquisser le niveau des risques encourus par ces développements informatiques. 
Une étude de ce type avait déjà été menée, pour un groupe européen d'assurance, la Skandia Insurance Company, par l'Institute For Future (U.S.A.). La structure de cette étude a été conservée dans ses grandes lignes; la comparaison entre les résultats obtenus se trouve en Annexe E; les différences sensibles observées tiennent, selon nous, à deux facteurs principaux :

- un écart de quatre années dans la réalisation des enquêtes;

- un échantillon entièrement européen dans le cas présent, alors que celui de l'étude de Skandia était en quasi totalité d'origine américaine.

\subsection{Objectifs de la recherche}

Le développement de l'utilisation des ordinateurs, rendu de plus en plus indispensable dans la société moderne, comporte une série de dangers inhérents au fonctionnement défectueux, aux pannes, aux violations du système (fraude, malveillance)... qui peuvent se traduire par:

- des pertes économiques par erreurs de calcul ou de régulation, fraudes financières, pertes de ressources, coût de réparation, des erreurs, pertes de production ;

- des pertes intangibles*: atteinte à la vie privée, pertes d'informations, pertes de clients, etc.

La mission de recherche avait donc pour objectifs principaux, sur le plan européen et à l'horizon 1988 :

1. d'identifier les principales applications potentielles de l'utilisation des ordinateurs;

2. d'évaluer les risques économiques encourus par les utilisateurs du secteur privé et public ;

3. de proposer une série de palliatifs envisageables;

4. et, parmi ces palliatifs, de déterminer quels pourraient être les points d'application essentiels pour les compagnies d'assurance (produits nouveaux ou modifications de produits).

\subsection{Résumé des conclusions}

L'étude prospective a été menée grâce à la collaboration active d'un groupe de 40 experts européens (voir Annexe F). Les principales conclusions du travail effectué s'attachent à :

- définir le cadre général de la pénétration de l'informatique en Europe dans la prochaine décennie ;

- analyser les risques encourus et évaluer les pertes potentielles concomitantes.

\footnotetext{
* A noter que certains de ces intangibles peuvent et doivent être traduit en termes économiques.
} 
1.2.1. Hypothèse de travail : Développement de l'utilisation de l'informatique

- La croissance du recours à l'informatique pour traiter l'information dans les différents secteurs économiques, techniques, sociaux et publics se poursuivra dans les douze prochaines années. En Europe de l'Ouest, de 1500 ordinateurs installés en 1960, à 55000 équipements en 1973, les prévisions s'élèvent à 450000 systèmes installés en 1988.

- La valeur du parc informatique installé en Europe de l'Ouest passera de 1,6 en 1960, à 40,8 en 1973, puis à 200 milliards de francs suisses à l'horizon 1988.

- Le ratio Ventes annuelles en informatique / Produit National Brut évoluera de 1,6\% en 1970 à 1,85 en 1973, puis devrait se situer vers $6 \%$ en 1988.

Tableau 1. Prévisions de l'évolution du parc ordinateurs

Nombre des ordinateurs installés

\begin{tabular}{|c|c|c|c|c|c|c|}
\hline & 1960 & 1970 & 1973 & 1978 & 1983 & 1988 \\
\hline $\begin{array}{l}\text { U.S.A. } \\
\text { Europe de l'Ouest } \\
\text { Japon } \\
\text { Autres pays }\end{array}$ & $\begin{array}{r}5500 \\
1500 \\
400 \\
1600\end{array}$ & $\begin{array}{r}65000 \\
21000 \\
6000 \\
18000\end{array}$ & $\begin{array}{r}110000 \\
55000 \\
19000 \\
46000\end{array}$ & $\begin{array}{r}200000 \\
110000 \\
45000 \\
95000\end{array}$ & $\begin{array}{r}400000 \\
225000 \\
70000 \\
205000\end{array}$ & $\begin{array}{l}700000 \\
450000 \\
140000 \\
460000\end{array}$ \\
\hline Total & 9000 & 110000 & 230000 & 450000 & 900000 & 1750000 \\
\hline $\begin{array}{l}\text { Pour le monde, } \\
\text { les minis en } \%\end{array}$ & $\varepsilon$ & 40 & 60 & 70 & 85 & 90 \\
\hline
\end{tabular}

- Cette croissance est légèrement supérieure à celle prévue pour les Etats-Unis, mais cependant inférieure au taux escompté au Japon ainsi que dans les pays du COMECON.

- Les principaux impacts techniques et technologiques des douze prochaines années peuvent se résumer ainsi :

- abaissement d'un ordre de grandeur, au moins, du ratio coût-productivité des équipements ;

- mise au point de grands réseaux de transmission d'informations traitées en temps réel avec saisie et restitution des données sur un nombre élevé de terminaux simples (écrans cathodiques, par exemple) ;

- utilisation extensive de mini ordinateurs, connectés ou non, à ces réseaux : en $1965,12 \%$ des ordinateurs installés étaient de petit taille; en 1988, ils formeront $90 \%$ du parc installé ;

- les techniques d'utilisation, programmation en particulier, feront des progrès sensibles, mais un réel progrès dans ce domaine viendra de l'utilisation de technologie câblée. 
- Les événements socio-économiques qui peuvent accélérer ce scénario de développement de l'informatique seront basés, en particulier, sur :

- la concentration des entreprises ;

- la modification du style de vie ;

- l'accroissement sensible du coût de la main-d'œuvre.

- Par contre, on peut prévoir que, notamment sur le plan social, les écueils rencontrés pourront fortement influer sur les prévisions faites. Les principaux facteurs freins tiendront à la réticence des individus devant les contraintes apportées par l'utilisation de l'informatique, devant les dangers de pertes de confidentialité (banque de données individuelles) et, en général, devant l'image de l'informatique comme outil technocratique figé. Le rejet de l'informatisation pourrait alors prendre une forme violente qui se traduirait par des fraudes (syndrome de Robin des Bois) et des sabotages.

- Dans les douze prochaines années, on assistera à une vitesse de développement relativement inégale dans les différents domaines des applications informatiques: rapide dans les systèmes de type indusltriel (contrôle des processus de fabrication), moins élevée dans les systèmes de gestion et, enfin, plus lente dans les applications de type public et social.

\subsubsection{Risques encourus et pertes potentielles résultantes}

Parmi 41 applications envisageables à l'horizon 1988, les experts en ont retenu 20 qui sont susceptibles d'être achevées dans les termes fixés.

- Avec toutes les réserves concernant l'évaluation de pertes économiques, traduisant un arrêt d'exploitation, des pertes commerciales voire des atteintes à la santé ou à la vie humaine, les estimations globales concernant les dommages économiques annuels causés par des incidents divers dans les systèmes informatiques ont été évalués à 6,6 milliards de francs suisses.

- Un peu plus de $70 \%$ de ces pertes sont le fait des applications de gestion; $12 \%$ découlent des systèmes de contrôles de processus industriels, et $15 \%$ sont imputables aux applications de type social et public.

- La fréquence annuelle des incidents varie de quelques-uns par an (sabotage ou erreurs graves de programmation) à 3 millions (erreurs dans la saisie des informations comptables) avec une médiane à 800 F.S.

- L'amplitude par incident va d'une centaine à 28 millions de F.S. (programmes erronés dans le contrôle de fabrication en pharmacie) avec une valeur médiane de 120000 F.S.

- Les dommages affectent directement en grande partie les entreprises utilisatrices d'informatique; cependant, les clients, ainsi que les personnes, forment une part importante des sinistres.

- Les incidents s'avèrent de types très variés; cependant plus de $40 \%$ des pertes sont causées par des erreurs directes des utilisateurs des systèmes informatiques (fausses ou incomplètes informations fournies aux systèmes, entre autres). Les pannes ou le mauvais fonctionnement des divers équipements forment $1 / 3$ des pertes; de 
mauvaises méthodes d'utilisation (programmation incorrecte) $15 \%$; fraudes et sabotages entrent enfin pour $10 \%$ dans le total des pertes estimées.

- L'analyse des experts a enfin permis de mettre en évidence un besoin de s'attaquer globalement au problème de la sécurité générale des systèmes informatiques. L'ampleur des risques encourus requiert une prise de conscience des utilisateurs et la définition de produits généraux, notamment dans les formes d'assurances responsabilité civile et pertes d'exploitation, particulièrement adaptées aux dangers développés par les systèmes informatiques des années 80 .

\section{Domaines de l'utilisation future de l'informatlque}

\subsection{Généralités}

Dans la période qui sert de référence à cette étude : 1973-1988, il est prévu une expansion considérable de l'utilisation de l'informatique. Cette progression considérable peut être schématisée par quelques-uns des chiffres retenus dans les développements estimés sur quinze années :

- le nombre des équipements installés dans le monde sera multiplié par 7,5 soit une croissance moyenne annuelle de $14 \%$;

- la valeur du parc ordinateurs installé, pendant la même période, sera un peu plus que quadruplée (croissance moyenne annuelle de $10 \%$ );

- alors que l'évolution du PNB devrait être de 2,5\% par an en moyenne aux EtatsUnis, et de 2,75\% en Europe Occidentale.

Il s'agit donc de la continuation de la pénétration rapide de l'informatique dans les structures économiques et sociales de notre monde moderne, malgré un ralentissement du développement économique général. Cette croissance se fera :

- dans des domaines connus actuellement tels que la gestion des entreprises, l'automatisation des processus industriels ;

- mais aussi dans le secteur des applications de type public et social: santé, gestion publique, enseignement.

Pour mieux cerner les problèmes rencontrés dans cette étude prospective nous avons donc divisé les principaux domaines d'utilisation de l'informatique en trois grandes parties bien identifiées :

Applications de gestion : c'est-à-dire tout ce qui concerne le traitement de l'information dans les entreprises: industries, distribution, et organismes financiers et administratifs (banques, assurances).

Contrôle et régulation des processus de type industriel: automatisation plus ou moins complète de la production de biens et de produits ainsi que les utilisations de l'informatique dans la distribution physique de certains de ces produits (carburants, par exemple).

Applications de type public et social qui ont trait aux problèmes tournés vers l'homme, soit à tìtre individuel (médecine, par exemple), soit à titre collectif (administration publique, etc.). 
Les 41 systèmes, composant l'éventail de départ des applications potentielles de l'informatique dans les quinze prochaines années, ont été sélectionnés par un groupe de travail interne et décrits en termes généraux avant d'être soumis aux jugements des experts composant le panel de consultation. Ces 41 applications montraient une large variété de solutions techniques :

- ordinateur autonome traitant les informations par lots : cas de la paye et de la comptabilité des entreprises, des applications de gestion publique locale;

- ordinateur travaillant en temps réel: système de marketing, contrôle de processus industriels, systèmes d'enseignement ;

- réseaux d'ordinateurs interconnectés: alerte météorologique générale, système boursier ;

- banques de données médicales ou individuelles en liaison directe avec les utilisateurs.

\subsection{Sélection des applications}

La première étape de cette étude devait donc permettre de choisir, parmi les 41 applications proposées aux répondants, ainsi que parmi les suggestions qui furent faites par les membres du panel, celles qui avaient le plus de chances de se réaliser à une échelle importante (de manière à induire un potentiel de pertes économiques), avant la fin de la période considérée, c'est-à-dire 1988.

La méthode employée fut la méthode Delphi, basée comme dans tous les processus d'études prospectives à long terme, sur les opinions des experts concernant la date à laquelle chacune des applications devait fonctionner dans les limites fournies par la définition. Par exemple : au moins $90 \%$ de toutes les entreprises européennes dont les effectifs dépassent 200 personnes ont recours à l'informatique pour effectuer leurs travaux de paye et de comptabilité.

La première série d'enquêtes fut compilée en affectant à chaque réponse un coefficient de pondération qui tenait compte du fait que le répondant était expert, familier ou peu averti de chacune des applications étudiées. Les résultats furent renvoyés aux membres du panel afin qu'ils puissent, pour chaque application, modifier leur réponse initiale s'ils le jugeaient utile.

En même temps le panel travaillait sur les événements socio-économiques capables d'accélérer ou de freiner la date moyenne de réalisation prévue pour chaque application. Dans une première étape, les répondants identifièrent les événements socio-économiques graves dont l'impact pouvait modifier, en général, la pénétration de l'informatique. Ensuite il fixèrent des limites générales à l'impact de ces événements, puis enfin, dans une troisième étape, définirent les limites de ces modifications possibles au niveau de chaque application. Le tableau 2 montre les résultats de l'analyse globale, par événement, alors que, en Annexe A, figurent, par application, les problèmes spécifiques posés par ces événements socio-économiques traduits également en termes de date au plus tôt et de date au plus tard.

La synthèse de tous ces éléments a permis de déterminer les applications qui ont au moins 50 chances sur cent d'être développées dans les termes définis au départ pour la période considérée.

Le tableau 3 montre les différentes applications retenues et celles non retenues par le panel réuni pour cette étude. 


\section{Tableau 2 -}

INF LUENCE DES EVENEMENTS SOCIO-ECONOMLUES

SUR LA VITESSE DE PENETRATION DE L'INFORMATIQUE

\begin{tabular}{|c|c|c|c|c|c|c|c|c|}
\hline \multirow{2}{*}{$\begin{array}{c}\text { EVENEMENTS } \\
\text { SOCIO-ECONOMTQUES }\end{array}$} & \multirow[b]{2}{*}{-2ans } & \multicolumn{3}{|c|}{ FREINS } & \multicolumn{4}{|c|}{ ACCELERATEURS } \\
\hline & & 3-5ans & 6ans & moyenne & $1-2$ ans & $3-5$ ans & 6ans & moyenne \\
\hline $\begin{array}{l}\text { 1. Aggravation de la réces- } \\
\text { sion économique et du chomâ- } \\
\text { ge. }\end{array}$ & 49,8 & 43,4 & 6,8 & 3 ans & 88,2 & 5,9 & 5,9 & 2 ans \\
\hline $\begin{array}{l}\text { 2. Règlement concernant l'u- } \\
\text { tilisation de l'informatique }\end{array}$ & 51.0 & 40.0 & 9.0 & 3 ans & 73.6 & 26.4 & - & 3 ans \\
\hline 3. Rejet de l'informatisation & 53.0 & 23.5 & 23.5 & 4 ans & - & - & - & - \\
\hline $\begin{array}{l}\text { 4. Réduction du niveau des dé } \\
\text { penses militaires et spatiales }\end{array}$ & 50.8 & 40.6 & 8.6 & 3 ans & - & - & - & - \\
\hline $\begin{array}{l}\text { 5. Reglementations européen- } \\
\text { nes concernant l'industrie in- } \\
\text { formatique non européenne. }\end{array}$ & 50.0 & 45.5 & 4.5 & 3 ans & - & - & - & - \\
\hline $\begin{array}{l}\text { 6. Obligation d'assurance con } \\
\text { tre les dommages causés par } \\
\text { l'informalique. }\end{array}$ & 61.2 & 24.5 & 14.3 & 2 ans & - & - & - & - \\
\hline $\begin{array}{l}\text { 7. Echecs et insuffisances du } \\
\text { système actuel d'enseigne- } \\
\text { ment. }\end{array}$ & 33.3 & 42.4 & 14.3 & 4 ans & 61.5 & 30.8 & 7.7 & 3 ans \\
\hline $\begin{array}{l}\text { 8. Règlementation concernan } \\
\text { les informaticiens (code de } \\
\text { déontologie). }\end{array}$ & 94.7 & 0 & 5.3 & $?$ ans & - & - & - & - \\
\hline $\begin{array}{l}\text { 9. Tendance générale de modit } \\
\text { cation du style de vie. }\end{array}$ & 71.4 & 21.5 & 7.1 & 2 ans & 56.1 & 31.5 & 11.9 & 4 ans \\
\hline $\begin{array}{l}\text { 10. Plus grande participation } \\
\text { des salariés et des syndicats } \\
\text { à la vie de l'entreprise. }\end{array}$ & 52.6 & 42.1 & 5.3 & 3 ans & - & - & - & - \\
\hline $\begin{array}{l}\text { 11. Concentration des entre- } \\
\text { prises. }\end{array}$ & & & & & 39.7 & 43.5 & 16.8 & 4 ans \\
\hline $\begin{array}{l}\text { 12. Accroissement du secteur } \\
\text { nationalisé et du controle. }\end{array}$ & & & & & 53.2 & 39.2 & 7.6 & 3 ans \\
\hline $\begin{array}{l}\text { 13. Formation a l'utilisation } \\
\text { des ordinateurs. }\end{array}$ & & & & & 54.3 & 41.8 & 3.9 & 4 ans \\
\hline $\begin{array}{l}\text { 14. Augmentation du coût de } \\
\text { la main d'oeuvre. }\end{array}$ & & & & & 35.0 & 36.0 & 26.0 & 4 ans \\
\hline $\begin{array}{l}\text { 15. Rejet de tout travail méca } \\
\text { nique et fastidieux. }\end{array}$ & & & & & 41.2 & 35.3 & 23.5 & 3 ans \\
\hline 16. Intégration européenne & & & & & 47.0 & 41.2 & 11.8 & 4 ans \\
\hline
\end{tabular}


Tableau 3.

Retenues par le Panel

Non retenues par le Panel

Gestion

- Paye et comptabilité

- Cartes de crédit

- Gestion automatique des transactions

- Conduite des réunions à distance bancaires

- Gestion production assemblage

- Système boursier

- Systèmes d'aide à la décision et aux prévisions

- Système de marketing

- Informatique foncière

- Systèmes documentaires

- Traitement des textes

- Gestion du personnel

- Gestion de la distribution

Contrôle et régulation de processus

- Contrôle de processus dans les raffineries

- Contrôle de fabrication dans l'industrie alimentaire

- Régulation de la production et de la distribution d'énergie électrique

- Commande numérique des machinesoutils

- Contrôle de fabrication dans l'industrie pharmaceutique

- Contrôle de fabrication dans l'industrie chimique

- Conception automatique des produits

- Préparation et production des journaux

- Contrôle de fabrication dans l'industrie du pneu

- Contrôle de qualité des pièces et produits

- Régulation de la distribution des carburants

- Conception en ingénierie

Applications de type social et public

- Contrôle du trafic urbain

- Système d'alerte à la pollution

- Banque de données individuelles

- Alerte météorologique

- Gestion publique locale

- Laboratoires d'analyses médicales automatiques

- Contrôle du trafic aérien

- Système de contrôle des matières dangereuses

- Surveillance des maisons et immeubles

- Banque de données légales

- Diagnostic permanent en vol aérien

- Aide au diagnostic médical

- Systèmes d'enseignement individualisé

- Systèmes de surveillance médicale

- Aide aux soins médicaux 


\subsubsection{Applications de gestion}

- Au moins $90 \%$ de toutes les entreprises européennes dont les effectifs dépassent 200 personnes ont recours à l'ordinateur pour effectuer leurs travaux de paye et de comptabilité (limites 1980 et 1985, probablement vers 1982).

- Plus de $75 \%$ des transactions bancaires en Europe, comprenant les transferts clientbanque et banque à banque, seront enregistrées et traitées par un système informatique (entre 1983 et 1988, probablement vers 1985).

- Pour $1 / 3$ des usines européennes d'assemblage, système central automatisé de planification et de suivi de toutes les phases d'assemblage (entre 1983 et 1989, probablement vers 1985).

- Cotations en temps réel des valeurs et traitement immédiat des cotations sur toutes les places boursières européennes (limites 1980 et 1987 ; probablement vers 1982).

- Au moins 100 entreprises industrielles européennes auront en fonctionnement un système complet de marketing en temps réel comprenant une base donnée pour études prévisionnelles, administration des ventes, statistiques commerciales, contrôle des coûts distribution et études de stratégie commerciale (limites 1981 et 1989, probablement vers 1985).

- Au moins $30 \%$ des pools dactylographiques utilisant plus de trois personnes dans les entreprises européennes seront dotés de système de traitement de textes pour augmenter la productivité et rendre le travail plus agréable (limites 1979 et 1983; probablement vers 1981 ).

- Au moins $75 \%$ de toutes les chaînes de distribution avec plus de dix magasins en Europe auront un système de gestion intégrée comprenant : saisie des ventes en temps réel, gestion des stocks, réapprovision et gestion des fournisseurs pour les articles non périssables (limites 1981 et 1984 : probablement vers 1984).

\subsubsection{Contrôle et régulation de processus industriels}

- Toutes les raffineries de pétrole installées en Europe fonctionneront complètement sous le contrôle d'un système automatique (dates limites 1979-1985; probablement vers 1981).

- Plus de $25 \%$ des entreprises européennes de l'industrie alimentaire utiliseront un contrôle automatisé des processus de fabrication pour piloter et surveiller la qualité de la production et notamment la pureté des produits fabriqués, et ceci pour au moins la moitié de leurs produits (dates limites 1983-1988; probablement vers 1986).

- Plus de $50 \%$ des entreprises européennes de l'industrie pharmaceutique utiliseront pour tous leurs produits un système automatisé intégré pour contrôler et surveiller la qualité de la production (entre 1980 et 1987 ; probablement vers 1982).

- Au moins $65 \%$ des usines européennes de l'industrie chimique utiliseront un système automatisé intégré pour contrôler les processus de fabrication et surveiller la qualité de la production (entre 1981 et 1990 ; probablement vers 1983).

- Plus de $75 \%$ des usines de fabrication de pneumatiques en Europe utiliseront un système complet de contrôle des processus de fabrication (dates limites 1979-1988; probablement vers 1983). 
- Régulation en temps réel, et pour au moins $80 \%$ du réseau européen, de la distribution par pipelines des carburants (gaz, pétrole...) (dates limites 1980 et 1987 ; probablement vers 1984).

\subsubsection{Applications de type public et social}

- Dans au moins 15 grandes villes européennes, systèmes automatisés de régulation des transports de type privé par analyse systématique des différents flux de véhicules (dates limites 1982 et 1987 ; probablement vers 1985).

- Pour les centres urbains européens de plus de 250000 habitants, mesure des différents niveaux de pollution, prévision d'évolution à court et moyen terme $(2$ à 12 heures) et déclenchement d'alerte s'il apparaît que les limites de sécurité vont être dépassées (dates limites 1980 et 1985 ; probablement vers 1982).

- Banque de données à l'échelon national concernant les individus et incluant tous les renseignements administratifs possibles à l'usage de l'administration gouvernementale et locale (dates limites entre 1984 et 1990 ; probablement vers 1986).

- Système général mondial d'informations météorologiques et de prévision (au moins cinq jours à l'avance) des événements naturels graves (tremblements de terre, ouragans, raz de marée, etc.) (dates limites entre 1980 et 1988 ; probablement vers 1985).

- Au moins $75 \%$ des villes européennes de plus de 10000 habitants utiliseront un système de gestion (comptabilité, impôts, recensement, problèmes fonciers) basé sur ordinateur (dates limites 1984 et 1990 ; probablement vers 1985).

- Systèmes utilisés dans au moins $50 \%$ des laboratoires médicaux pour effectuer automatiquement des analyses chimiques (dosages d'urée sanguine) et des analyses physiques (EEC et EEG) (dates limites 1981 et 1989 ; probablement vers 1984).

- Plus de 25 écoles européennes de niveau supérieur auront un système d'enseignement individuel basé sur la méthode d'enseignement programmé avec contrôle individuel de la progression des connaissances (dates limites 1983-1990; probablement vers 1986).

\subsection{Raisons du choix fait par le panel d'enquêtes}

Pour chacune des applications retenues pour l'évaluation des pertes potentielles, les raisons du jugement fait par les répondants sur les facteurs favorables et défavorables à la mise en œuvre plus ou moins rapide de ces applications figurent en Annexe A.

La synthèse des raisons qui ont conduit le panel à choisir ces applications montre que :

- l'informatique est souvent citée comme le seul moyen d'aboutir aux systèmes complexes requis ;

- la sécurité des systèmes informatiques est le critère cité en second, aussi bien pour les applications de gestion (diminuer les erreurs humaines), que pour celles de contrôle de processus ou de type public et social;

Ensuite viennent les facteurs liés au problème de main-d'œuvre :

- accroissement des coûts,

- manque de personnel qualifié,

- réduction de la durée de travail ; 
- enfin, les facteurs liés à une réglementation nationale et parfois supranationale (CEE notamment) sur le contrôle de qualité, sur les marges bénéficiaires, sur l'environnement, etc.

2.4. Applications non retenues par le panel, mais qui présentent un haut degré de vraisemblabilité d'achèvement aux limites de la période d'étude retenue (1974-1988)

Les huit applications suivantes n'ont pas été retenues dans l'étude détaillée, cependant elles présentent, toutes, au moins $50 \%$ de chances d'être réalisées, dans les termes définis, dans les années immédiatement supérieures au terme fixé à notre étude : 1988.

\section{Dates}

- Cartes de crédit

- Systèmes documentaires, à vocation scientifique répondant au langage naturel

- Conception automatique des produits

- Préparation et production des journaux

- Contrôle trafic aérien

- Aide au diagnostic médical

- Aide aux soins médicaux

- Système de surveillance médicale
Au plus tôt

1988

1988

1987

1988

1989

1989

1987

1986
Au plus tard

1992

1992

1993

1993

1995

1994

1992

1991

\section{Etude des pertes envisageables}

\subsection{Sommaire}

Après une discussion générale sur les risques informatiques, discussion dont il faut extraire deux idées principales :

- une sous-évaluation considérable à l'heure actuelle des risques encourus par l'utilisation des systèmes informatiques, aussi bien pour les utilisateurs que pour l'industrie de l'assurance ;

- la difficulté de quantifier l'ensemble des pertes économiques potentielles en informatique.

Cette étude montre :

- une large variété d'incidents possibles (au moins 20 types);

- une gamme de fréquence d'incidents allant de 2 ou 3 incidents par an à plusieurs millions ;

- une amplitude financière par incident depuis une centaine de francs suisses jusqu'à 30 millions de francs suisses.

Le montant total annuel des pertes recensées peut s'élever à 6625 millions de francs suisses pour l'Europe. Compte tenu que toutes les pertes possibles n'ont pas été prises 
en compte ni toutes les applications informatiques étudiées en détail, il devrait être possible d'envisager que cette estimation ne représente qu'environ moitié des pertes totales imputables à l'utilisation des systèmes informatiques, à l'horizon 1988, en Europe.

- Deux tiers de ces pertes potentielles sont imputables aux conditions d'utilisation (15\% pour des erreurs de programmation, $40 \%$ pour des erreurs au niveau des hommes en contact avec les systèmes informatiques et $10 \%$ seraient dus à la fraude et au sabotage).

- Les pertes directes subies par les entreprises s'évaluent à environ $70 \%$ du montant total.

\subsection{Les risques informatiques}

Jusqu'à présent, l'assurance en informatique (voir en Annexe D quelques éléments détaillés) couvre les risques identifiés dans certains secteurs tels que :

- risques matériels (équipements, locaux, coût de reconstitution des fichiers, etc.);

- pertes d'exploitation ;

- responsabilité civile.

On peut cependant remarquer brièvement que :

- l'industrie de l'assurance semble sous-estimer, dans une très large mesure, le niveau et la fréquence des pertes potentielles inhérentes à l'utilisation de systèmes informatiques ;

- les usagers, quant à eux, ignorent en quasi-totalité les risques encourus. Nous avons été surpris de l'ampleur de ce problème en analysant les réactions des panelistes. Excepté ceux qui sont issus de l'industrie de l'assurance, pratiquement tous les autres, soit plus de $80 \%$ de l'échantillon retenu sur l'importance des objectifs de l'étude, ont été stupéfaits lorsque, au $3^{\mathrm{e}}$ questionnaire, ils ont été confrontés avec la quantification de la fréquence et de l'amplitude financière des pertes envisageables.

Il faut donc insister sur l'actuel manque d'informations exhaustives et précises sur la sécurité des systèmes informatiques et sur les risques encourus. Il semble qu'aucune documentation précise n'ait été rassemblée et que l'information des clients potentiels (utilisateurs d'informatique) ait été largement sous développée.

Quoi qu'il en soit, l'objectif de cette étude devait permettre, non pas d'évaluer le risque économique potentiel total actuel encouru en informatique, mais, pour chacune de quelque vingt applications principales existant en Europe à l'horizon 1988, d'isoler les pertes potentielles envisageables dans les quatre plus importants incidents.

Ces incidents peuvent se produire dans l'un quelconque des maillons constituant la chaîne d'un système informatique au niveau :

- de l'utilisateur (pris au sens général du terme : employé en relation directe avec le système informatique) soit, par exemple, en communiquant des données erronées ou incomplètes au système (code inversé, montants de transactions tronqués, etc.), soit en utilisant incorrectement les informations reçues par le système ; 
- des équipements :

- d'entrée en ordinateur (terminaux pour la demande de réservation de places d'avion, analyseurs automatiques dans un processus de régulation de raffinerie),

- de traitement, c'est-à-dire des ordinateurs centraux commandant le système (panne totale ou partielle),

- de restitution des informations (par exemple, écrans d'affichage des résultats, commande automatique des signaux, etc.);

- du logiciel ou software, c'est-à-dire des programmes écrits par l'entreprise ou l'organisme pour faire fonctionner son système informatique d'où généralement des erreurs de logique et/ou de compréhension des problèmes posés.

Par ailleurs, ces incidents peuvent être soit :

- fortuits et occasionnels (cas des pannes)

- ou provoqués (cas des fraudes et sabotages),

et affecter soit :

- le système lui-même, en le bloquant dans son fonctionnement (par exemple : arrêt de fonctionnement des équipements de transmission);

- fait plus grave, car beaucoup plus difficile à déceler, les informations produites par le système (programmes erronés ou altération d'informations pendant leur transmission, par exemple).

On conçoit donc que, pour les systèmes complexes prévus dans les années 80 , de nombreuses possibilités d'incidents peuvent exister; on peut les regrouper sous vingt rubriques différentes et il ne pouvait être question d'analyser et d'évaluer, pour toutes les applications informatiques envisageables à cette date, toutes les différentes possibilités et leurs incidences économiques, qui, soit dit en passant, peuvent parfois être d'une importance sans commune mesure avec la cause de l'incident. Un exemple nous montrera mieux cette distorsion entre la cause et le résultat :

- dans un système destiné à contrôler les conditions météorologiques, le déclenchement d'une fausse alerte, alors que le seuil d'alerte n'est pas atteint, peut amener une dépense injustifiée de 750000 francs suisses.

Bien entendu, il existe de nombreux moyens de remédier aux incidents dans les systèmes informatiques. Chacune des causes d'incidents peut être étudiée isolément et une solution appropriée peut facilement être conçue ; par exemple :

- doubler les différents équipements,

- tester très longuement les nouveaux programmes puis les faire fonctionner parallèlement à l'ancien système,

- saisir les informations deux fois, à deux postes de travail distincts et comparer chacune des informations.

Cependant l'écueil est ici double :

- sur le plan opérationnel, les délais de traitement peuvent être considérablement augmentés ;

- sur le plan économique, la justification de tels systèmes «doubles 》 n'est plus possible; les dépenses annuelles moyennes affectées à l'informatique par rapport au 
chiffre d'affaires en Europe, en 1975, s'élèvent, par exemple, pour des systèmes évolués du type de ceux décrits dans cette étude, à :

$2 \%$ dans l'industrie automobile,

$1,5 \%$ dans la distribution.

Prendre systématiquement les solutions de sécurité décrites ci-dessus conduirait, en fait, à augmenter les ratios de dépenses informatiques d'environ 60 à à $70 \%$. Dans un contexte économique normal, quelle est l'entreprise qui pourrait justifier de telles mesures?

Les concepteurs chargés de définir les systèmes informatiques sont donc amenés, à l'heure actuelle, à négliger plus ou moins le problème des risques informatiques. Dans certains cas, ces risques sont évalués rationnellement et les maillons les plus faibles de cette chaîne informatique sont protégés: on double par exemple l'unité centrale, ou, encore, l'on prévoit un système de secours capable d'assurer la survie minimale du système, pendant un certain laps de temps, avec un ordinateur plus faible. Ceci est de plus en plus pratiqué dans les systèmes informatiques de type industriel (régulation de processus continu de fabrication) et dans les systèmes où d'importants dommages peuvent être causés soit directement sur le plan économique (réservation de places d'avion d'un montant unitaire élevé) soit indirectement (dommages matériels et éventuellement corporels causés par une erreur dans un système de contrôle de trafic urbain). Les membres du panel ont donc été confrontés, dans ce domaine, à une double difficulté :

- se placer dans un contexte réaliste où la sécurité des systèmes informatiques réalisés en 1988 tenait compte des facteurs opérationnels et économiques discutés ci-dessus (c'est-à-dire, en fait, ne pas se baser sur une idéalisation du fonctionnement de futurs systèmes informatiques);

- isoler les incidents paraissant les plus importants, en fréquence et en amplitude, et quantifier ces évaluations y compris celles qui sont du plus pur domaine qualitatif (coût de la vie d'un être humain, d'une atteinte portée à sa réputation, préjudice subi par une entreprise pour mauvais service rendu à la clientèle, etc.).

En résumé, pour aboutir aux objectifs de cette étude, il aura été nécessaire de :

- sélectionner les applications potentielles réalisables en 1988 et porteuses de pertes potentielles importantes ;

- identifier les risques les plus importants, en fréquence et amplitude ;

- quantifier les pertes potentielles envisagées.

\subsection{Les procédures de travail}

L'étude s'est déroulée en trois phases d'enquêtes dont certaines ont été réalisées en parallèle sur plusieurs domaines, avec des panelistes différents.

\section{Phase 1. Sélection des applications}

Quarante et une applications, réparties en trois grands domaines (Gestion, Industriel, Type public et social), ont été proposées aux jugements des membres du panel.

A partir d'une description schématique de l'application fournie aux membres du panel (voir Fig. 1), les panelistes ont donné leur opinion en particulier sur les dates 
Fig 1

Tire

CONTROTE DE FABRICATION DANS LDIULSTHE DU PNFU.

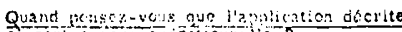

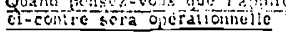

Meltre :

1075-1978

A : $10 \%$ de probiubilite

B : $50 \%$ de probabilite

$1978-1983$

$1383-1938$

C : $90 \%$ de probabilute

Plus tard

Jamals

Bases du raisonnenuent tilise

Inllis:ire aes uratements sucio-eculaningues

possities

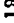

\section{Descripion teduraie :}

Plus de 75 q des usines de fabrication de preumatiquea en Eurque utiliseront un systeme complet de controle des processus de fabritation.

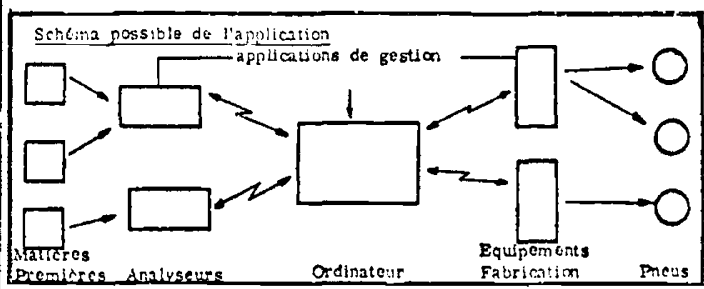

Quels sont les risques potentieis possibles?

Quel est votre ceste de ianilarite avec le sujet ?

Expert $\square$ Familier $\square$ Non tamilier $\square$

\begin{tabular}{c} 
TIITE: \\
REGULATION DE LA \\
DISTRIBUTION DES CARBURANTS \\
\hline
\end{tabular}

Q:and lonsez-vous nue l'anolication decrite

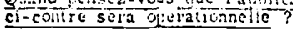

\section{Mettre :}

A : 10 s de probabilute

B : 50 \% de probabilite

C : 90 50 de prolszbilite

$1875-1978$

$1978-1583$

1933-1938

plus tard

Jamals

Bases du raiscriament utilise
옹

Descrifion zúncrale :

Regulation en temps réel, et pour au moins $80 \%$ du rếgeau eurapeen, de la distribution par pipeline des carburants (zas, petrole,....).

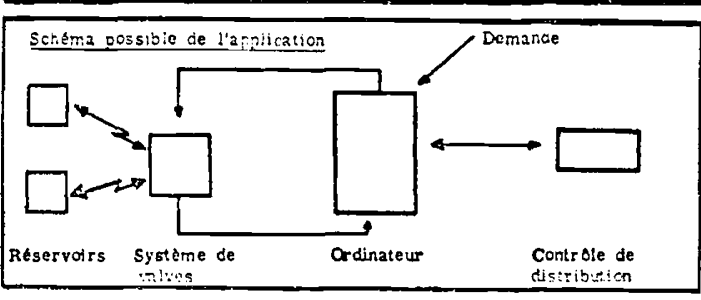

Quels scat les risques potentials pessibles?

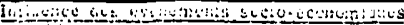
prenibies

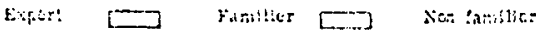


d'achèvement des applications, les bases de raisonnement utilisées, les risques potentiels possibles et le degré de familiarité avec le sujet étudié.

De plus, en même temps, étaient étudiés :

- l'impact de certains événements de nature socio-économique sur le développement prévisible de l'informatique;

- l'évolution des techniques informatiques et celle concomitante du développement du marché de l'informatique.

La sélection des applications retenues pour l'étude plus détaillée s'est faite sur deux critères :

- probabilité forte de fonctionnement de l'application dans la période 1974-1988,

- niveau des pertes potentielles estimées.

\section{Phase 2. Analyse détaillée des vingt applications retenues}

Pour chacune des vingt applications sélectionnées, complétées à la fois par les résultats obtenus par la première itération et par des données complémentaires destinées à fournir aux panélistes des informations permettant de réfléchir à nouveau sur le problème (voir Fig. 2), les nouvelles questions portaient sur :

- la modification des dates de réalisation de l'application;

- le choix des quatre plus importants incidents possibles (notion d'importance définie par l'amplitude et la fréquence des pertes).

En même temps était poursuivie l'analyse de l'impact des événements socio-économiques et l'étude des innovations techniques et technologiques.

Il est à remarquer que :

- $35 \%$ environ des panelistes ont modifié leur opinion sur les dates probables de réalisation des applications ;

- dans plus de trois quarts des cas cette modification s'est traduite par un resserrement de l'éventail des opinions (voir en annexe A le détail par application);

- les opinions concernant les quatre plus importants incidents possibles pour chaque application ont été remarquablement convergents et ceci quel que soit le type d'application (gestion, industriel, de type social et public). En général les incidents les plus importants ont recueilli plus de $60 \%$ des citations valides; les douze autres, soit trois fois plus en nombre, n'étant cités au total que dans une proportion de moitié environ $(35 \%)$;

- par contre, les opinions concernant la fréquence et l'amplitude des pertes se sont situées, comme il est assez normal dans l'étude d'un phénomène complexe aléatoire à long terme, dans une assez large fourchette : de 1 à 8 en moyenne.

Phase 3 : Itération sur la fréquence et l'amplitude des pertes potentielles

Le troisième questionnaire contenait des éléments concernant :

- l'évolution technologique et technique des équipements ; 
Figure 2 -

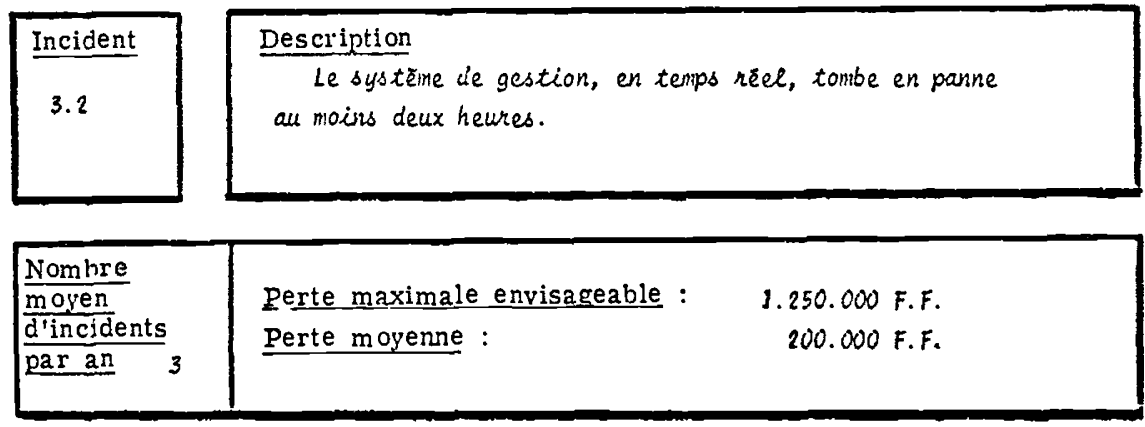

Qui sera le plus grand perdant ?: L'organisme de gestion du systeme, et L'utilisateur - point de vente.

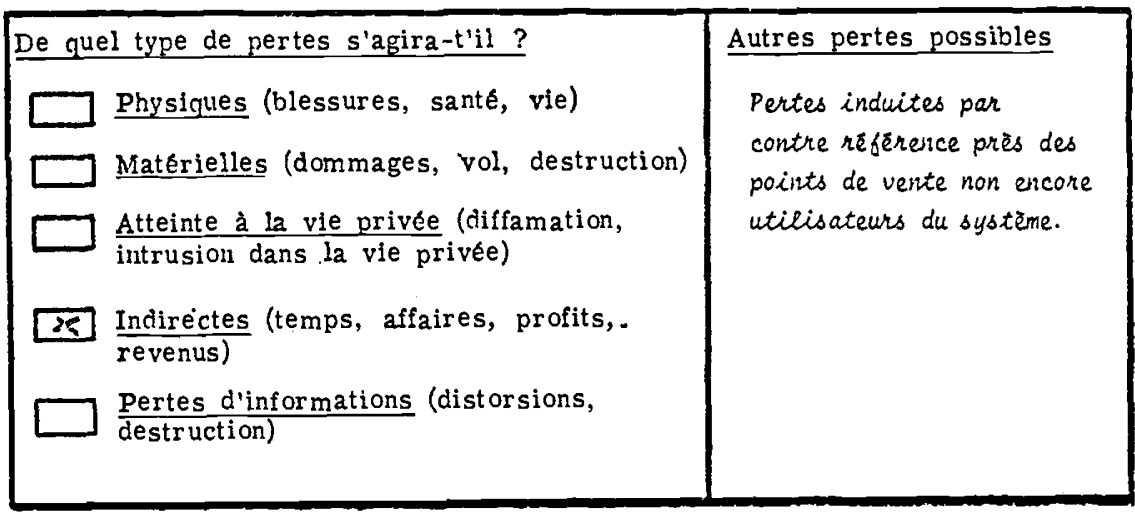

Par quels moyens peut-on minimiser les pertes encourues?

- Doubler le sustème informatiạe dans ses composartes les plus vulnerabes (transmission, unite centrale).

- Prévoir un système de dégradation double lpossibilite de traiter par lots. si le système en temps rêlel est bloquél.

- Prevoir une assurance type "perte d'exploitation". 
- les prévisions de développement du marché de l'informatique mises au point par un panel spécifique (voir Annexe B) ;

- l'impact estimé des événements socio-économiques sur la pénétration générale de l'informatique en Europe ;

- la synthèse des opinions émises précédemment par le panel sur la fréquence et l'amplitude des pertes.

Il était demandé, dans cette itération, de réfléchir à nouveau sur ces différents points et d'indiquer les changements d'opinions éventuels.

Il doit être noté ici que ces énumérations brèves ne montrent pas suffisamment l'importance du travail demandé ; certains documents d'enquêtes comptaient jusqu'à 80 pages de questions complexes et variées; il nous faut donc insister sur la gratitude que nous éprouvons envers ceux qui ont accepté de consacrer à cette étude, et leur temps, et leurs efforts.

\section{Synthèse}

Précédée d'une enquête directe pour permettre de préciser certains points ou apporter certains compléments aux informations obtenues, la synthèse réalisée a permis d'aboutir :

- aux informations plus détaillées incluses notamment dans les Annexes A, C et D ;

- aux résultats exposés dans la suite de ce chapitre.

Qu'il nous soit permis de souligner à nouveau que, dans une telle étude prospective, les différentes informations ne doivent être prises que comme des indicateurs généraux de tendance et d'opinion, indicateurs susceptibles d'attirer l'attention des responsables au plus haut niveau sur des phénomènes de grande ampleur, mais non de servir de base à des prévisions précises.

\subsection{Les pertes potentielles}

\subsubsection{Pertes potentielles totales}

\section{- Principe d'évaluation}

Pour chacune des vingt applications analysées en détail, les membres du panel ont d'abord identifié les quatre plus importants incidents envisageables (voir page 20, Procédures de travail, et Phase 2, Phase 3), puis estimé pour chaque incident :

- la fréquence annuelle,

- l'amplitude moyenne et maximale.

Nous avons donc obtenu une fréquence moyenne annuelle et une amplitude moyenne d'un incident. Les pertes potentielles envisageables pour un incident sont égales au produit de ces deux éléments (fréquence moyenne annuelle $X$ amplitude moyenne d'un incident).

La somme de ces pertes potentielles annuelles par incident a permis alors de calculer les pertes totales annuelles pour une application. 
Un exemple est donné dans le tableau ci-dessous :

Application $X$

\begin{tabular}{c|c|c|c}
\hline $\begin{array}{c}\text { Principaux } \\
\text { incidents }\end{array}$ & $\begin{array}{c}\text { Fréquence } \\
\text { annuelle } \\
\text { moyenne }\end{array}$ & $\begin{array}{c}\text { Amplitude } \\
\text { moyenne } \\
\text { (en 000 F.S.) }\end{array}$ & $\begin{array}{c}\text { F } \times \text { A } \\
\text { (en 000 F.S.) }\end{array}$ \\
\hline A & 100 & 1 & 100 \\
\hline B & 5 & 10 & 50 \\
\hline C & 10000 & 0,3 & 3000 \\
\hline D & 25 & 12 & 300 \\
\hline
\end{tabular}

Pertes totales pour l'application :

- Résultats

$$
100+5 \cdot 10^{4}+3 \cdot 10^{3}+300 \text { soit } 53,4 \text { millions de francs suisses }
$$

Les vingt applications sélectionnées présentent un potentiel total de pertes annuelles estimé à 6625 millions de francs suisses.

Les Figures 3 et 4 montrent sous forme d'une liste et d'un graphique les pertes potentielles pour chacune des vingt applications retenues dans l'échantillon d'étude détaillée.

Ces différentes applications ont été regroupées en trois grands domaines :

Gestion : 7 applications

Contrôle de processus : 6 applications

Type social et public : 7 applications
$73 \%$ du total des pertes

$11,5 \%$ du total des pertes

$13,5 \%$ du total des pertes

Ces chiffres appellent trois commentaires importants :

- ils ne représentent pas toutes les pertes totales par application, puisque seuls les quatre incidents les plus importants ont été analysés ; on peut penser que, pour avoir une idée des pertes totales par application, les résultats ci-dessus peuvent être majorés d'environ un tiers ;

- la perte globale pour tous les systèmes mis en œuvre à l'horizon 1988 est nettement plus importante : seules vingt applications ont été analysées, soit sans doute un tiers, au maximum un demi, du potentiel total informatique mis en place à cette date. Pour raisonner en terme de "marché » en tenant compte des remarques ci-dessus, on peut sans doute multiplier le potentiel total évalué dans l'étude (6 625 millions de francs suisses) par un facteur 2;

- les bases de calcul sont basées sur une estimation raisonnable faite par quarante experts européens ; comme toutes les hypothèses à long terme, elles ne peuvent être considérées que comme des indicateurs de tendance. 
Figure 3. Pertes potentielles annuelles par applications

\section{GESTION}

Paye et comptabilité

2260

Gestion automatique des transactions bancaires

164

Gestion production assemblage

736,5

Système boursier

453

Système de marketing 266,9

Traitement des textes 386

Gestion de la distribution

CONTROLE DE PROCESSUS INDUSTRIEL

Contrôle de processus dans les raffineries 234

Contrôle de fabrication dans l'industrie alimentaire 115,4

Contrôle de fabrication dans l'industrie pharmaceutique 104,2

Contrôle de fabrication dans l'industrie chimique 150

Contrôle de fabrication dans l'industrie du pneu 86,2

Régulation de la distribution des carburants 63,2

\section{APPLICATIONS DE TYPE PUBLIC ET SOCIAL}

Contrôle du trafic urbain 277

Système d'alerte à la pollution

Banque de données individuelles

Alerte météorologique

Gestion publique locale 254,6

Laboratoires d'analyses médicales automatiques 130,6

Systèmes d'enseignement individualisé 


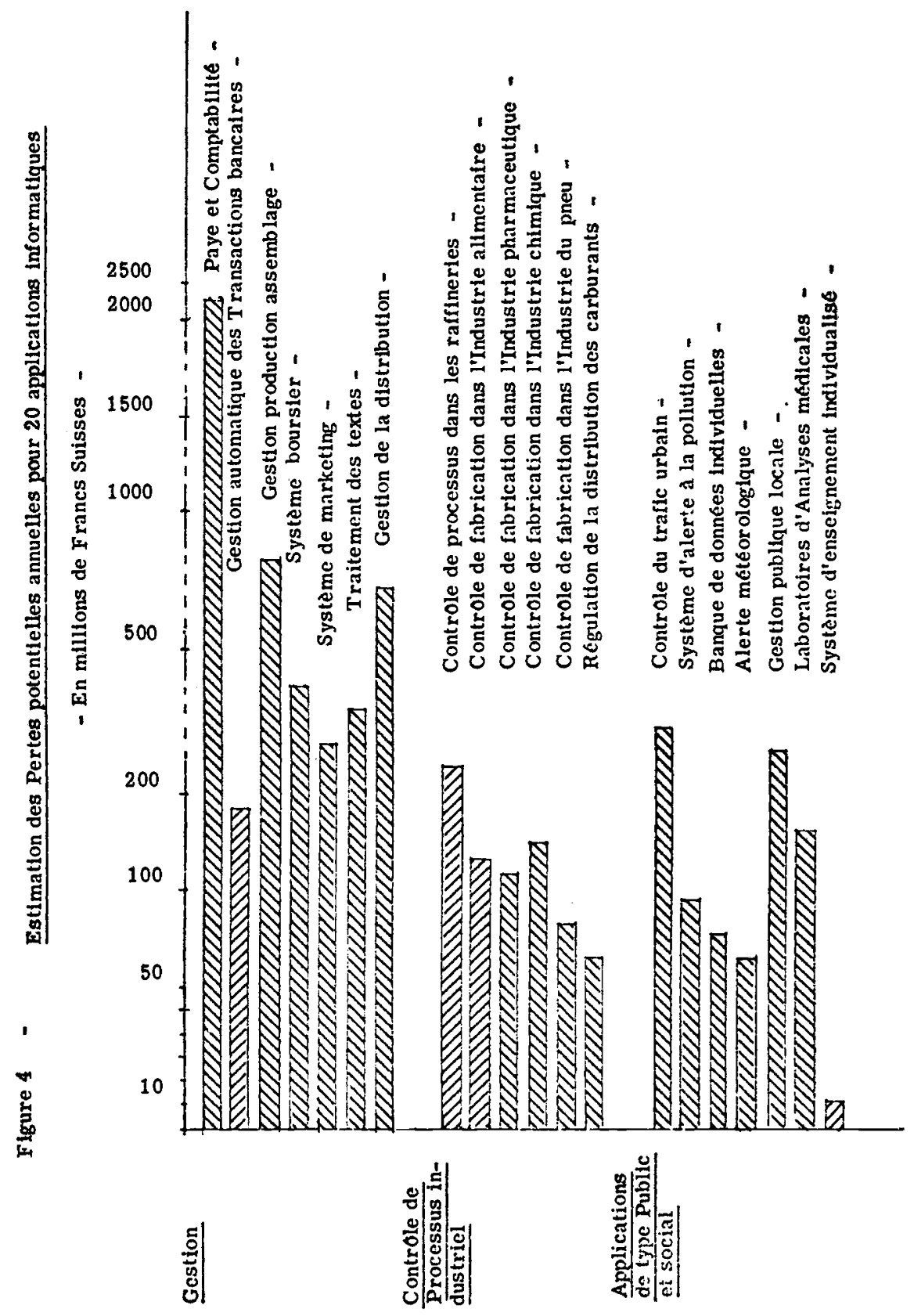


3.4.2. Analyse par niveau de pertes potentielles

Pour chacune des applications étudiées nous avons séparé les pertes qui étaient égales ou supérieure à 50 millions de francs suisses par incident et celles qui étaient inférieures à ce chiffre.

La Figure 5 montre le détail de cette analyse.

On peut considérer que :

- les applications de gestion ont généralement des pertes par incident très substantielles (de 71 à $100 \%$ des pertes annuelles par incident sont égales ou supérieures à 50 millions de francs suisses). Ceci tient particulièrement aux faits que les systèmes informatiques de ce type traitent de nombreuses informations (d'où une fréquence moyenne annuelle d'incidents très élevée) et que chaque incident peut avoir une valeur unitaire relativement élevée ;

- les applications de contrôle de processus montrent que $55 \%$ des incidents peuvent occasionner des pertes égales ou supérieures à 50 millions de francs suisses, en général avec une valeur unitaire élevée (arrêt de la production) mais une fréquence moindre que dans le cas précédent ;

- le domaine des applications de type social et public montre une hétérogénéité remarquable des appréciations ; certaines qui sont voisines des applications de gestion (gestion publique par ex.) ont la même allure que celle-ci $(92 \% \geqslant 50$ millions de francs suisses); d'autres au contraire se situent à l'opposé : cas des banques de données individuelles et des systèmes d'enseignement individualisé, etc., peut-être parce qu'il a été plus difficile, dans ces cas, de traduire, sur le plan économique, les préjudices moraux causés par des défaillances de ces systèmes.

Figure 5. Etude du niveau des pertes potentielles annuelles par applications

Applications informatiques

\section{GESTION}

- Paye et comptabilité

- Gestion automatique des transactions bancaires

- Gestion production assemblage

\begin{tabular}{c|c}
\multicolumn{2}{c}{$\%$ des pertes } \\
supérieur & $\begin{array}{c}\text { inférieur } \\
\text { à } 5 \times 10^{7}\end{array}$ \\
à $5 \times 10^{7}$ \\
\hline 90 & 10 \\
100 & - \\
80 & 20 \\
71 & 29 \\
94 & 6 \\
93 & 7 \\
90 & 10
\end{tabular}




\section{CONTROLLE PROCESSUS}

- Contrôle de processus dans les raffineries

- Contrôle de fabrication dans l'industrie alimentaire

- Contrôle de fabrication dans l'industrie pharmaceutique

- Contrôle de fabrication dans l'industrie chimique

- Contrôle de fabrication dans l'industrie du pneu

- Régulation de la distribution des carburants

\section{PUBLIC ET SOCIAL}

- Contrôle du trafic urbain

- Système d'alerte à la pollution

- Banque de données individuelles

- Alerte météorologique

- Gestion publique locale

- Laboratoire d'analyses médicales automatiques

- Systèmes d'enseignement individualisé

Moyenne générale

\begin{tabular}{r|r}
65 & 35 \\
79 & 21 \\
- & 100 \\
53 & 47 \\
92 & 8 \\
55 & 45 \\
- & 100 \\
69 & 31
\end{tabular}

\subsubsection{Fréquence et amplitude des pertes}

\section{Principes de calcul}

Les évaluations montrent une combinaison des incidents envisageables telle que on conçoit de nombreux incidents annuels (plusieurs millions dans les cas du traitement de textes) pour une faible valeur (coût de rectification) ou une fréquence annuelle faible (deux sabotages des systèmes de contrôle de production dans les raffineries), avec une valeur unitaire élevée ( 28 millions de francs suisses pour les erreurs de programmation en contrôle de production pharmaceutique).

Parce que, pour l'utilisateur, la prévention des incidents, et que, pour l'industrie des Assurances, l'évaluation des risques dépendent de la fréquence et de l'amplitude estimées des incidents, nous avons cherché à définir la fréquence et l'amplitude médiane des incidents étudiés.

La figure 6 montre la répartition des estimations faites au niveau de chaque incident par les membres du panel:

Fréquence médiane

Amplitude médiane
800 incidents/an

120000 F.S./incident 
Figure 6. Définition: fréquence et amplitude des pertes

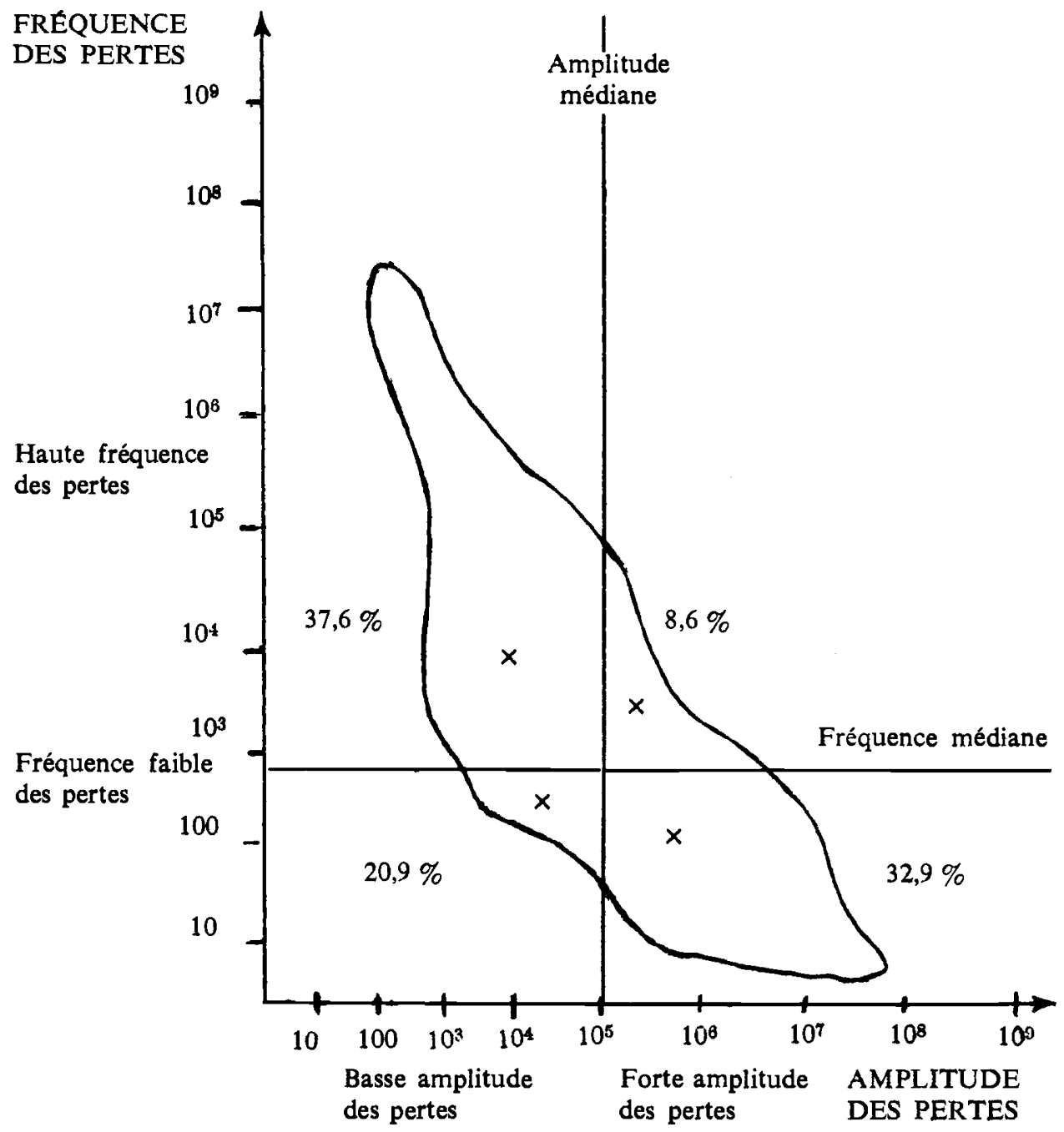

- La fréquence des pertes est indiquée en nombre d'incidents par année.

- L'amplitude des pertes est indiquée en francs suisses par incident.

- Les pourcentages indiquent le nombre de toutes les estimations faites pour tous les incidents se rangeant dans les quatre zones.

$X$ représente le point central des estimations de chacune des quatre zones. 
La figure 7 montre les comparaisons faites sur les trois domaines d'application étudiés chacun en fonction de l'importance des pertes réparties en quatre zones:

Fréquence Faible / Basse Amplitude

Fréquence Faible / Forte Amplitude

Haute Fréquence / Basse Amplitude

Haute Fréquence / Forte Amplitude

Il est intéressant de noter que :

- dans les trois domaines d'application, ce sont les incidents du type «Haute Fréquence - Basse Amplitude » qui prédominent, très nettement moins accusé dans le contrôle des processus ;

- c'est dans le domaine des applications de contrôle de processus que les incidents du type "Fréquence Faible - Forte Amplitude» sont les plus développés (presque aussi importants que ceux du type "Haute Fréquence - Basse Amplitude »);

- les estimations faites sur les deux autres types d'incidents sont notoirement plus faibles, notamment pour "Forte Amplitude - Haute Fréquence », qui se trouve pratiquement d'une importance marginale.

L'analyse détaillée de chacune des applications par rapport à son classement dans les quatre zones définies précédemment montre qu'en réalité chacune d'entre elles comprend, quasi naturellement, plusieurs natures d'incidents. Nous avons recherché cependant les prédominances de chacune d'entre elles pour essayer de les regrouper par nature de risque.

Applications à risque limité : Fréquence Faible / Basse Amplitude.

Les incidents sont estimés peu nombreux (en nombre inférieur à la fréquence médiane) et chacune des pertes ne dépasse par 120000 francs suisses.

Dans ce secteur se retrouvent plusieurs applications de type public et social :

- banques de données individuelles;

- systèmes d'enseignement individualisé ;

- à un degré moindre: laboratoires d'analyses médicales automatiques (y a-t-il une sous-estimation des dommages causés à la clientèle ?)

Ainsi que l'application de régulation de la distribution des carburants.

Applications à haut risque: Haute Fréquence / Forte Amplitude.

Ces applications, caractérisées par des incidents nombreux, dont chacun peut se traduire par une perte importante, sont celles qui, en général, peuvent avoir un impact direct sur l'être humain pendant sa vie courante. C'est donc le cas de certains incidents des applications de contrôle de processus dans :

- l'industrie alimentaire,

- l'industrie pharmaceutique,

- l'industrie chimique. 

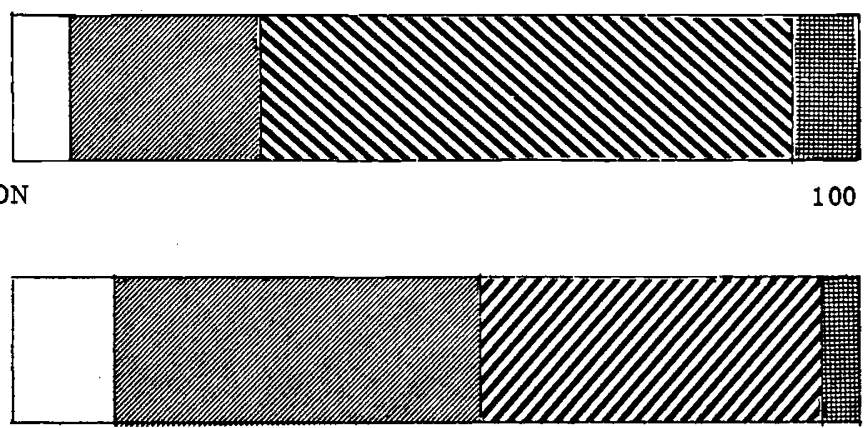

CONTROLE PROCESSUS

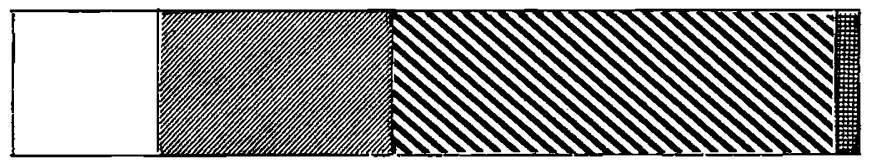

APP. TYPE PUBLIC ET SOCLAL

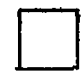

F. Faible

B. Amplitude

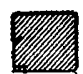

F. Faible

F. Anplitude

\section{$\mathbb{Z}$}

Hte fréquence

F. Amplitude

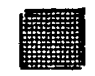

Hte fréquence F. Amplitude 
Applications à haut risque aléatoire: Faible Fréquence / Forte Amplitude.

De telles situations, qui sont en nombres relativement importants, se présentent surtout dans :

- le domaine financier: gestion bancaire, système boursier ;

- le domaine du contrôle des processus en général (pannes graves, sabotages, erreurs de conception des programmes...).

Quelques applications de type social et public: alerte à la pollution et alerte météorologique...

Les pertes, dans ce secteur, sont donc de type aléatoire et leur prédiction est difficile; cependant leur impact peut être considérable puisque le niveau de perte de chaque incident (peut-être encore sous-estimé dans l'étude) est estimé très élevé.

\section{Applications de Forte Fréquence / Basse Amplitude:}

Les estimations prévoient, ici, des incidents nombreux, mais d'amplitude inférieure à la médiane. Le cas type prévoit des erreurs de saisie des informations, des pannes simples qui se traduisent par des couts de correction des erreurs, quelques pertes légères d'exploitation, et/ou de production. Dans ce secteur, on retrouve principalement des applications de :

- gestion : assemblage, marketing, traitement de textes, distribution.

\subsubsection{Type de dommages}

De la notion de pertes, caractérisée par sa fréquence et son amplitude, il faut passer à l'identification des différents types de dommages puisqu'en réalité les estimations économiques faites dans cette étude dépendent étroitement de l'évaluation du préjudice subi.

Et si dans certains cas ce préjudice est mesurable directement (coût de correction d'une erreur en comptabilité, par ex.) ou quasi directement (perte de production d'une journée dans une usine d'assemblage), le processus est beaucoup plus difficile et complexe lorsqu'il s'agit d'évaluer économiquement la dégradation soit de l'image d'une entreprise, soit du service rendu à la clientèle, sans parler des atteintes à la santé voire à la vie humaine.

Les types de dommages ont été classés en quatre catégories suivant leur impact immédiat :

- hommes (vie, santé, réputation...),

- entreprises utilisatrices (pertes directes),

- clients (produits défectueux, pertes de marchés),

- divers (pertes d'informations, de confidentialité...).

Il est évident que cette classification, comme toutes celles qui auraient pu être adoptées, doit bien être considérée dans son aspect instantané et que, en réalité, les dommages causées sont pratiquement toujours imputables (et imputés) à l'entreprise, 
ou l'organisme, qui a généré le dommage (... avant que celui-ci ne fasse intervenir son assureur...). Prenons deux exemples :

- des dommages matériels (ou corporels) causés par le mauvais fonctionnement d'un système de régulation du trafic urbain sont, en définitive, imputés à l'organisme chargé d'assurer la circulation ;

- des produits chimiques dérectueux, pour une erreur du système informatique de contrôle de production, causent d'abord un dommage au client... qui se retourne vers son fournisseur...

La figure 8 montre la ventilation des pertes potentielles globales par type de dommages. Elle montre clairement que, dans l'utilisation de l'informatique, la couverture de l'entreprise utilisatrice doit comprendre tout l'ensemble des préjudices possibles.

La figure 9 présente une comparaison des différents dommages par type d'application. On peut noter que, suivant le domaine d'application, les pertes affectent différemment les différents types de dommages.

En gestion, $95 \%$ des pertes sont des préjudices directs (pertes d'exploitation notamment, pertes de clientèle, dépenses d'exploitation supplémentaire) et affectant les clients. Les dommages "Hommes》 et "Divers》 (généralement pertes d'informations confidentielles) ne représentent qu'une part extrêmement faible (5\% du total).

Figure 8. Pertes annuelles totales par type de dommages

$$
\begin{gathered}
\text { Pertes annuelles } \\
\text { estimées } \\
\text { (en millions de F.S.) }
\end{gathered}
$$

Hommes

Entreprise

Clients

Autres
550

4800

900

370

6620 millions de FS
Pourcentage sur

Pertes totales

8,5

72,4

13,5

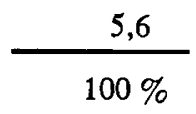

- Dans le domaine du contrôle de processus, on note un accroissement sensible $(18,5 \%$ du total) des pertes affectant les problèmes humains, en particulier sur le plan de l'intégrité physique (santé, vie). Les dommages relatifs à la clientèle s'accroissent également ( $23 \%$ du total).

- Dans les applications de type public et social, les dommages éventuels concernant l'homme représentent un tiers des pertes estimées; tous les aspects sont alors touchés : au niveau corporel (défaillances dans les systèmes de régulation du trafic urbain) aussi bien qu'au niveau moral (divulgation diinformations confidentielles, altération d'informations officielles...). 


\section{Figure 9}

\section{Pertes Annuelles Estimées}

Par type d'Application

et Par type de Dommages

$$
0
$$

$100 \%$

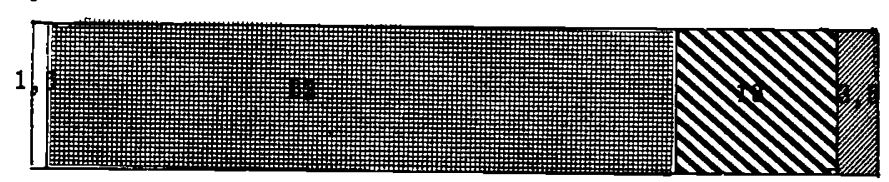

GESTION

$100 \%$

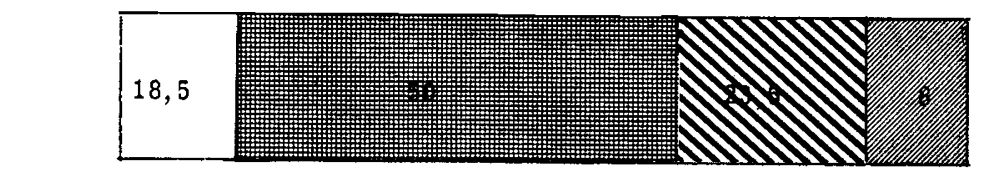

CONTROLE PROCESSUS

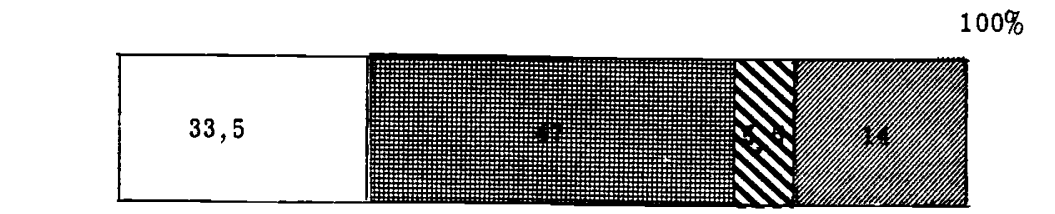

PUBLIC et SOCIAL
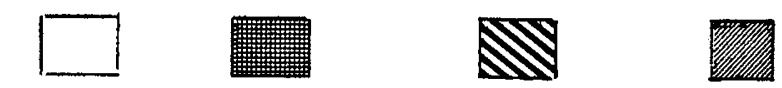

Personnel

Entreprise

Clients

Autres 


\subsection{Type d'incidents :}

- Principe d'analyse

Le deuxième questionnaire envoyé aux membres du panel proposait vingt types d'incidents possibles; dans un premier temps les répondants devaient identifier les quatre incidents les plus importants, en hiérarchisant leur choix, puis évaluer la fréquence et l'amplitude de ces incidents. Après synthèse et sélection, pour chaque application, des quatre incidents majeurs, le troisième questionnaire permettait une itération sur les opinions exprimées précédemment.

Enfin, chacun des incidents évalué au niveau d'une application a été reclassé dans quatre grandes catégories basées sur l'origine de l'incident.

\section{- Résultats}

La figure 10 montre, pour toutes les applications étudiées, la répartition des pertes totales par type d'incidents.

Par ordre décroissant d'importance relative on trouve donc:

— utilisation du système

$41,8 \%$

- équipements

$32,9 \%$

- méthodes d'utilisation

$15,2 \%$

- fraude - sabotage

Figure 10. Pertes totales annuelles par type d'incidents

1. Equipements

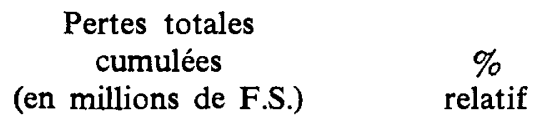

- Ordinateurs et réseaux

- Terminaux et matériels saisie

- Terminaux de restitution

$\frac{455}{2180} \quad \frac{6,9}{32,9}$

2. Méthodes d'utilisation

- Logiciel

$\begin{array}{r}710 \\ 300 \\ \hline 1010\end{array}$

- Exploitation

4. Divers

- Fraudes - Sabotages

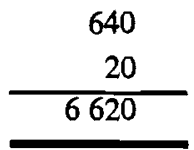

- Autres 
La figure 11 présente, pour les trois grands domaines d'application étudiés, l'importance relative des pertes annuelles ventilées par type d'incidents.

Pour les applications Gestion, les incidents causés par les erreurs des utilisateurs forment $56 \%$ du total des pertes. Par contre, dans le domaine des applications de contrôle de processus industriels, où les systèmes sont fortement automatisés sans grande intervention de l'homme puisque la saisie des informations est faite automatiquement par capteurs, $91,4 \%$ des incidents sont causés par les équipements ou leurs méthodes d'utilisation (programmes, exploitation). Enfin c'est dans le domaine des applications du type public et social que les méthodes d'utilisation et la fraude/sabotage sont relativement plus élevés que dans la moyenne.

\section{Erreurs des utilisateurs (41,8\% des pertes).}

En général, il s'agit d'erreurs commises lors de la saisie des informations (des données fausses ou altérées sont entrées dans le système) ou lors de l'utilisation des résultats fournis par le système, en particulier s'il y a intervention manuelle à ce stade ; par exemple, des informations sont affichées sur écran et l'utilisateur recopie (sur un billet d'avion, sur une fiche) certaines de ces informations. Ce type d'incident est donc fréquent dans les applications de gestion (plus de la moitié des pertes annuelles dans ce secteur) avec des pointes extrêmes dans:

- paye et comptabilité ( $85 \%$ des pertes),

- traitement de textes (78\% des pertes),

- gestion de la distribution (90\% des pertes),

et rencontré, quoique à un degré moindre, dans les applications de type public et social; $27 \%$ des pertes en moyenne avec un autre type d'incident; la régulation du trafic urbain est perturbée parce que basée sur des informations statistiques erronées (47\% des pertes), de même qu'une mauvaise utilisation des systèmes d'enseignement individualisé conduit à $45 \%$ de pertes imputables à l'utilisateur.

Parmi les diverses méthodes proposées pour prévenir les incidents, il faut insister sur :

- la formation du personnel et sa sensibilisation à l'intérêt du travail ;

- l'amélioration des conditions de travail pour le personnel en relation avec l'ordinateur, c'est-à-dire contenu affectif du dialogue homme-machine : nécessaire réaménagement des postes de travail;

- mise au point de méthodes diverses pour détecter et corriger les erreurs avant que l'information entrée dans le système soit traitée ;

- définition de circuits de contrôle spécifiques avec utilisation de certains résultats isus du système informatique (contrôle permanent par sondages);

- pour certaines applications (contrôle de processus, régulation du trafic...), simulation préalable au fonctionnement du système, et longue période de fonctionnement en 


\section{Figure 11-}

\section{Types d'Incidents par Domaines d'Application}
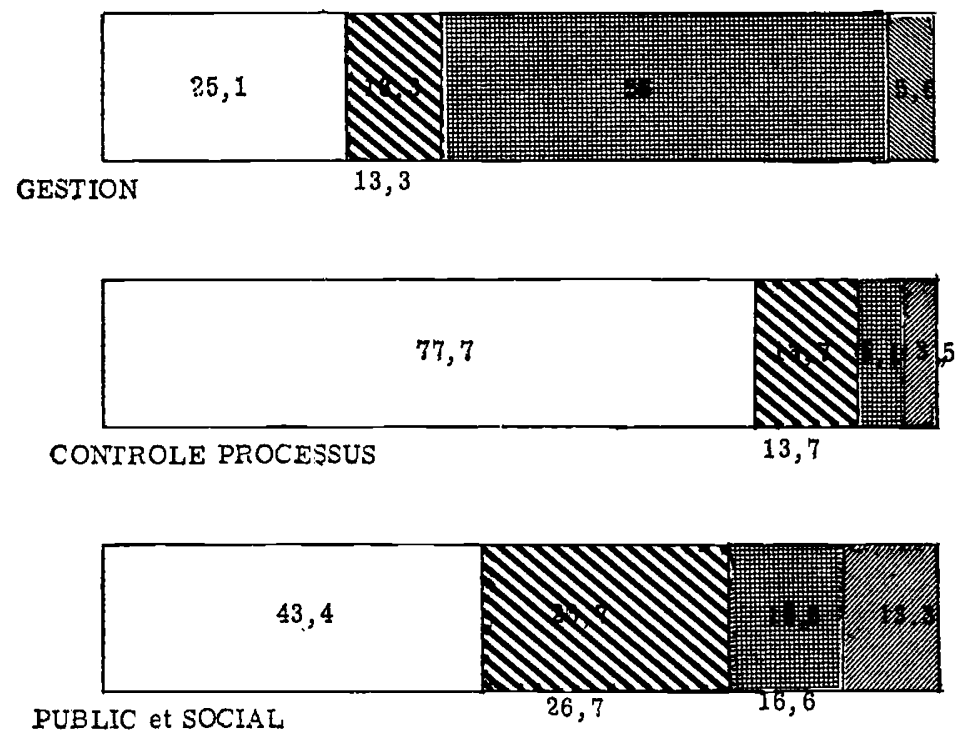

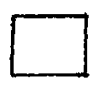

Equipements

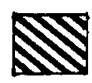

Méthode Utilisation

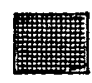

Utilisateurs

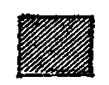

Fraude-Sabotage 
double, ancien/nouveau système, pour s'assurer que les informations de base sont correctes.

Aucune référence au recours à certaines formes d'assurance relevée dans ce domaine.

Equipement (32,9\% des pertes)

$\begin{array}{lr}\text { soit Terminaux et matériel de saisie } & 18,7 \% \\ \text { Ordinateurs et réseaux } & 7,3 \% \\ \text { Terminaux de restitution } & 6,9 \%\end{array}$

Les incidents les plus fréquemment cités ressortent des pannes ou arrêts complets des divers dispositifs composant le système informatique ou des fonctionnements défectueux de façon systématique ou fugace (plus difficile à détecter).

Plus le système est fermé, c'est-à-dire plus il fonctionne sans recours à l'homme (cas des applications de contrôle de processus industriels) et plus l'importance de ce type d'incidents croit :

$\begin{array}{ll}\text { Contrôle processus } & 77,7 \% \text { des pertes. } \\ \text { Public et Social } & 43,4 \% \\ \text { Gestion } & 25,1 \%\end{array}$

Parmi les cas les plus typiques :

Contrôle de processus des raffineries $\quad 90 \%$

Contrôle de processus industries alimentaires $\quad 87 \%$

Contrôle de processus chimique $\quad 90 \%$

Contrôle de processus pneumatiques $\quad 100 \%$

ainsi que, dans le domaine Secteur Public et Social, les applications dont la nature se rapproche des systèmes de contrôle de processus, en particulier les systèmes de monitoring et d'alerte (pollution, météorologie).

Les mesures proposées pour réduire les pertes se classent en quatre grandes catégories :

- doublement des équipements, ou des parties d'équipements, les plus fragiles et/ou exposées ;

- maintenance préventive et procédures rapides de dépannage ;

- définition de procédures de secours: travail manuel ou système en dégradation douce (graceful degradation) et leur surveillance systématique par audit extérieur;

- assurances : pertes d'exploitation dans $90 \%$ des cas; responsabilité civile de façon beaucoup moins fréquente.

Méthodes d'utilisation (15,2\% des pertes):

avec deux grandes catégories :

$\begin{array}{ll}\text { logiciel } & 10,7 \% \\ \text { exploitation } & 15,2 \%\end{array}$


Les deux types d'incidents concernent donc :

- soit des erreurs de conception dans les programmes fournis au système informatique :
système boursier
$55 \%$ des pertes
système de marketing
$34 \%$ des pertes
contrôle pharmaceutique
$54 \%$ des pertes
banque données individuelles
$20 \%$ des pertes
alerte à la pollution
$82 \%$ des pertes

- soit des erreurs au niveau de l'exploitation du système : utilisation de logiciel périmé, erreurs de manipulation, utilisation de fichiers incorrects.

Parmi les méthodes de réduction des pertes, on peut citer :

- contrôle strict des règles d'organisation de la production ordinateur ;

- formation poussée du personnel d'exploitation;

- assurance perte d'exploitation ;

- simulation approfondie des fonctionnements du nouveau système et tests sévères pendant un long fonctionnement en parallèle (six mois) avec l'ancien système ;

- recours systématique à un audit extérieur.

\section{Fraudes et Sabotages : $9,8 \%$}

L'annexe $\mathrm{C}$ est consacrée à une analyse de ce problème, d'autant plus préoccupant que l'on constate une accélération importance du phénomène en informatique (comme d'ailleurs en règle générale, puisque de 1969 à 1972 le taux des sinistres dus à la malveillance a augmenté dans le monde de $25 \%$ ).

Les "crimes informatiques», suivant la terminologie utilisée, s'attaqueraient surtout :

- aux applications de type financier, Banque et Bourse $(30,5 \%$ des pertes dans les systèmes bancaires) ;

- aux points sensibles des applications de type industriel : contrôle de la régulation de la distribution des carburants $(10 \%)$;

- à ce qui fournit un terrain de controverse à l'utilisation de l'informatique dans le domaine social et public.

$$
\begin{array}{ll}
\text { Banque de Données Individuelles } & 73 \% \\
\text { Systèmes d'Enseignement Individualisé } & 24 \%
\end{array}
$$

Ainsi détaillées dans l'annexe $\mathrm{C}$, la prévention et la détection sont onéreuses et doivent être constamment modifées. Les mesures doivent combiner des moyens techniques et technologiques (équipements spéciaux, logiciel), légales (à créer), sociales et méthodologiques (audit systématique). 


\section{Réflexlons sur les risques informatlques}

Cette étude aura nettement mis en évidence que :

1. Les risques de pertes existent à un haut niveau pour les systèmes informatiques et qu'ils vont aller croissant dans les prochaines années au fur et à mesure de la pénétration de l'informatique dans les entreprises et dans les systèmes économiques et sociaux de notre monde, pénétration qui va de pair avec la sophistication progressive de ces systèmes informatiques.

2. La prévention et la correction (au sens large du terme) des incidents est possible mais coûteuse et doit faire intervenir un ensemble de techniques, de méthodes et de fonctions décisionnelles: c'est le type du "risk management» qui doit être conçu comme un système complet impliquant :

Direction générale.

Directions fonctionnelles :

- celles qui utilisent l'informatique,

- celles qui sont responsables des fonctions administratives et financières.

Spécialistes :

- informaticiens,

- auditeurs,

- prévention technique (feu, fluides, divers, alimentation électrique, etc.),

- sociologues et socio-psychologues,

- formateurs,

- constructeurs d'informatique.

Pouvoirs publics.

Associations professionnelles.

3. A l'heure actuelle, non seulement il n'existe que très peu de responsables conscients de ces problèmes mais, fait plus grave encore, ni l'information ni les canaux de diffusion de cette information n'existent (ou bien sous forme incomplète, fragmentaire donc, en définitive inadaptée aux nécessités analysées).

Il apparaît évident que l'industrie de l'assurance doit se saisir du problème global et préparer dès à présent une série de propositions et de mesures concrètes avant que d'y être obligée par une pression politique brutale issue d'un (ou d'une série) sinistre particulièrement grave mettant éventuellement en danger quelque fragment d'une économie nationale.

Les risques informatiques apparaissent comme une partie (plus ou moins grande suivant le secteur économique, le degré d'informatisation, les mesures de prévention 
prises...) du problème du «risk management» d'une entreprise. Et il importe de préparer son identification et son traitement comme tel :

- définir les limites d'un modèle général ;

- préciser les différentes composantes du modèle; les produits, leur interpénétration et leur complémentarité ;

- se doter des structures d'attaque du marché ;

- recruter et former les collaborateurs nécessaires ;

- sensibiliser, puis informer et former les utilisateurs.

L'étude a montré qu'il était nécessaire d'agir sur un ensemble de facteurs :

- technologiques :

- augmentation de la fiabilité des équipements,

- possibilité d'utiliser ces équipements en procédures de secours,

- doublement de certains dispositifs (mémoires, lignes de transmission, unité de calcul...) des systèmes ;

- techniques :

- procédures de secours éprouvées incluant équipements en surnombre et logiciel capable de maintenir un niveau minimal d'activité du système ,

- maintenance rigoureuse et, autant que possible, préventive,

- méthodes actives (par programmes enregistrés) de détection et correction des erreurs et anomalies,

- procédures éprouvées de démarrage de nouvelles applications;

- sociaux et humains :

- former les utilisateurs en contact avec le système informatique,

- changer les méthodes actuelles de conception des systèmes informatiques qui doivent être plus axés sur le bonnes conditions de travail pour l'utilisateur;

- de contrôle :

- audit systématique et fréquent sur le fonctionnement du système ;

- légaux :

- définition de règles d'utilisation des systèmes informatiques et mise au point de certaines protections (informations, fichiers);

- économiques :

- investissements nécessaires dans la sécurité des systèmes informatiques (actuellement, notion totalement négligée) ; 
- protection économique (voir Annexe D) :

- Assurance Responsabilité civile,

- Assurance Perte d'exploitation,

- Assurance Equipements,

- Assurance Données et fichiers

Cependant, il faut noter que, de l'avis d'une très large majorité d'experts consultés, il ne faut pas compter pour résoudre le problème des risques informatiques ni sur les autorités politiques, ni sur les responsables d'entreprise, ni sur les constructeurs d'informatique et probablement pas beaucoup sur les informaticiens et l'industrie de l'assurance. Peut-être l'occasion est-elle belle d'infirmer leur prédiction sur ce tout dernier point?

Pour préciser quelque peu leur position, on peut écrire que :

- les experts pensent que le secteur du Risque Informatique sera couvert par un type sociétés de services qui seront mieux à même d'influer sur la conception initiale des systèmes, de mettre en place des méthodes générales de contrôle, de vérifier la permanence des mesures de sécurité (audit)... et de proposer un système général d'assurance "taillé sur mesure ». A la réflexion, il paraît quand même difficile d'imaginer une entitée supplémentaire dans le «système 》: clients - constructeurs assurances; de plus, le niveau des investissements nécessaires dépasse probablement la capacité financière des plus grandes sociétés de services informatiques actuelles, sauf si l'on envisage une participation extérieure;

- la pression des demandeurs (du marché) deviendra telle que le problème du Risque Informatique exigera une action vers 1982; en particulier sous l'influence de la concentration des entreprises (événement socio-économique favorable à l'accélération de la pénétration de l'informatique) et de l'accroissement de la taille des systèmes mis en œuvre;

- jusqu'à cette date, les experts croient que le Risque Informatique en tant que produit global ne sera proposé que par quelques compagnies d'Assurances, faisant œuvre de pionniers mais s'assurant ainsi une précieuse expérience et une avance importante ;

- enfin, les opinions émises par les membres au panel convergent sur quelques points qu'il est nécessaire de souligner :

- les plus grands dangers pour la société informatisée des années 1980 viendront du rejet (en cours de développement) des méthodes de traitement de l'information telles que développées actuellement. Sabotages, et fraudes, prendront de l'extension mais probablement sous une forme plus élaborée qu'aujourd'hui, donc d'autant plus dangereuse;

- les facteurs de type politique (intégration européenne, réglementation européenne concernant les constructeurs non européens) ne joueront qu'un rôle marginal dans la vitesse de pénétration de l'informatique en Europe ; 
- les risques de type classique (incendie, dégâts des eaux), qui n'ont pas été pris en compte dans cette étude, doivent continuer, comme actuellement, à être assimilés à des risques industriels .

$\mathrm{Au}$ terme de cette étude, il apparaît clairement que l'industrie des assurances peut être à la base de la prise de conscience des dangers que fera peser sur le monde économique, mais aussi dans le domaine social, le développement accéléré de l'utilisation de l'informatique. Inéluctablement, cette prise de conscience devra être suivie de la mise en ouvre des moyens propres à permettre un fonctionnement à la fois efficace et harmonieux de la société de la prochaine décennie.

Figure 12. Evénements socio-économiques classés par importance estimée

\section{FACTEURS ACCÉLERATEURS}

1. Concentration des entreprises

2. Modification du style de vie

3. Accroissement du coût de la main-d'œuvre

4. Intégration européenne

5. Réglementation concernant l'utilisation de l'informatique

6. Développement du secteur nationalisé en Europe

7. Formation intensive à l'utilisation de l'informatique

8. Aggravation de la récession

\section{FACTEURS FREINS}

1. Rejet de l'informatisation

2. Réglementation concernant l'utilisation de l'informatique

3. Modification du style de vie

4. Plus grande participation des salariés et des syndicats dans la gestion de l'entreprise

5. Insuffisances et échecs des systèmes actuels d'éducation

6. Réduction des dépenses militaires et spatiales

7. Déontologie des informaticiens

8. Obligation d'assurances contre les dommages informatiques

9. Réglementation européenne concernant les constructeurs d'informatique non européens

10. Aggravation de la récession 


\section{Annexe A: Evaluation des pertes possibles et des méthodes de réduction dans}

les vingt secteurs d'application sélectionnés

Cette annexe présente les résultats détaillés de l'étude pour chacune des vingt applications retenues par le panel d'experts.

Chaque application présente plusieurs parties :

- sur la première page :

- description de l'application,

- estimation de la date de réalisation moyenne prévue,

- impact estimé des événements socio-économiques possibles,

- facteurs pouvant accélérer ou freiner la réalisation de ce type d'application ;

- sur la deuxième page :

- pertes totales estimées,

- pourcentages de ces pertes atteignant 50 millions de francs suisses,

- fréquence et amplitude des pertes,

- répartition par nature des dommages causés,

- répartition des pertes par sources d'incidents,

- analyse détaillée des quatre types d'incidents les plus fréquents avec estimation de la fréquence annuelle et de l'importance moyenne d'un seul incident et suggestions faites pour réduire ces pertes. 


\section{APPLICATION N 1 PAYE ET COMPTABILITE -}

Au moins $90 \%$ de toutes les entreprises européennes dont les effectifs dépassent 200 personnes, ont recours à l'ordinateur pour effectuer tous leurs travaux de paye et de comptabilité.

\section{ESTIMATIONS DU PANEL}

DATE DE REALTSATION MOYENNE

$1 9 7 5 - 1 9 7 8 \longdiv { 2 }$

1978-1983

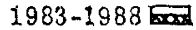

Aprés 1988

Jamais
Moyenne 1982

$1^{\circ}$ réponse

$2^{\circ}$ et $3^{\circ}$ réponses
DATES AU PLUS TOT

AU PLUS TARD

EN FONCTION DES EVENEMENT SOCIO ECONOMIQUES

Au plus tót 1980

Au plus tard 1985

\section{COMMENTAIIRES}

- Facteurs pouvant accelérer la date de réalisation.:

- Augmentation du coût de la main d'oeuvre supérieure à celle du prix des produits.

- Formation intensive à l'utilisation de l'informatique.

- Diminution demandée de la durée de travail et des travaux fastidieux.

- Facteurs pouvant freiner la date de réalisation :

- Rejet de l'utilisation de l'informatique sous sa forme actuelle.

- Prolongation de la crise économique actuelle.

- Plus grande participation des salariés à la vie de l'entreprise. 


\begin{tabular}{|c|c|c|c|}
\hline \multicolumn{4}{|c|}{ ANALYSK DEZ YERTES YOBSTLLS } \\
\hline 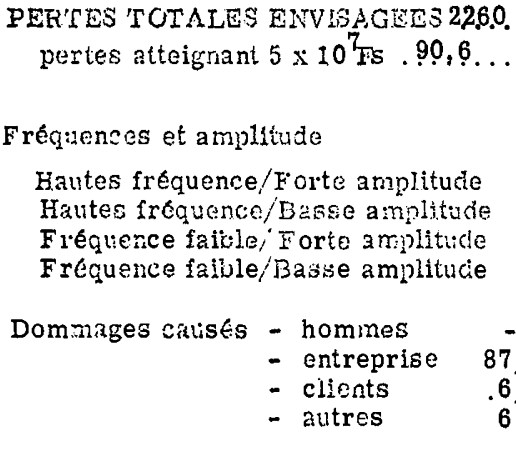 & \begin{tabular}{l|l} 
millions & S \\
$\ldots \ldots \%$ & \\
& \\
4,1 & \\
64,3 \\
30,6
\end{tabular} & $\begin{array}{l}\text { Sources dincidents } \\
\text { - Equipements } \\
\text { - Lcrictel } \\
\text { - Saisie } \\
\text { - Restitution } \\
\text { - Erreurs utilisateurs } \\
\text { - Fraude - sabotrge } \\
\text { - Divers (exploitation) }\end{array}$ & $\begin{array}{l}8,1 \\
- \\
84,7 \\
2,7 \\
4,5\end{array}$ \\
\hline \multicolumn{2}{|c|}{\begin{tabular}{c|c} 
Principules sources d'incidents & $\begin{array}{c}\text { Frequence } \\
\text { et amplitu- } \\
\text { de moyenne } \\
\text { des incidento }\end{array}$
\end{tabular}} & \multicolumn{2}{|c|}{$\begin{array}{l}\text { Proposition de linethodes } \\
\text { de réduction des pertes }\end{array}$} \\
\hline $\begin{array}{l}\text { Erreurs dans la fourniture et dans } \\
\text { l'utilisation d'informations entrées } \\
\text { et sorties des systemes se traduia } \\
\text { sant par: } \\
\text { - grèves, arrets de travail (paye) } \\
\text { - reparations pécuniaires envers les } \\
\text { employés penalisés. } \\
\text { - cout de correction des erreurs }\end{array}$ & $\begin{array}{c}2000 \\
\text { par an } \\
150.000 \mathrm{FS}\end{array}$ & \multicolumn{2}{|c|}{$\begin{array}{l}\text { - Systèmes de controle très complets } \\
\text { à l'entrée et à la sortie } \\
\text { - Formation des utilisateurs d'infor- } \\
\text { mations } \\
\text { - Controle permanent des systèmes }\end{array}$} \\
\hline $\begin{array}{l}\text { Erreur de manipulation des divers } \\
\text { fichiers et programmes : utilisation } \\
\text { des fichiers et programmes caducs. } \\
\text { - pertes de temps } \\
\text { - coats de correction des erreurs } \\
\text { - dédommagements des tiers. }\end{array}$ & $\begin{array}{l}500 \text { par an } \\
200.000 F S\end{array}$ & $\begin{array}{l}\text { - Controle précis des } r \\
\text { production ordinateur } \\
\text { - Formation des exploi } \\
\text { dinateur } \\
\text { - Augmentation de la sé } \\
\text { veau des systèmes op } \\
\text { nis par le constructeu }\end{array}$ & $\begin{array}{l}\text { ntes de } \\
\text { arite au ni- } \\
\text { atoires four- }\end{array}$ \\
\hline $\begin{array}{l}\text { Falsification delibérée des program- } \\
\text { mes pour des gains frauduleux : } \\
\text { - pertes financières directes } \\
\text { - cout de remise en ordre du sys- } \\
\text { tème. }\end{array}$ & $\begin{array}{l}20 \text { par an } \\
3 \text { millions } \\
\text { FS. }\end{array}$ & $\begin{array}{l}\text { - Audit systématique de } \\
\text { d'information, au mo } \\
\text { - Sondages de vraisemb } \\
\text { les programmes. } \\
\text { - Système de controle } \\
\text { plication. }\end{array}$ & $\begin{array}{l}\text { systèmes } \\
\text { s } 1 \text { fois/an. } \\
\text { nce dans } \\
\text { oisé par ap- }\end{array}$ \\
\hline $\begin{array}{l}\text { Erreur dans la saisie des informa- } \\
\text { tions : } \\
\text { - perte ou altération d'informations. } \\
\text { - coat de correction des erreurs }\end{array}$ & $\begin{array}{l}3 \text { millions } \\
\text { par an. } \\
600 \text { FS }\end{array}$ & $\begin{array}{l}\text { - Programmes de contr } \\
\text { entrées dans l'ordina } \\
\text { - Amélioration des équi } \\
\text { saisie. }\end{array}$ & $\begin{array}{l}\text { les avant } \\
\text { ur } \\
\text { ments de }\end{array}$ \\
\hline
\end{tabular}


Plus de $75 \%$ des transactions bancaires en Europe, comprenant les transferts client-banque et banque à banque, enregistrées et traitées par un système informatique.

\section{ESTTIMATIONS DU PANEY}

DATE DE REALISATION MOYENNE

1975-1978

1978-1983

$1983-1988$

Apr'es 1988

Jamais

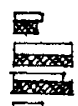

$=$
Moyenne 1985

DATES AU PLUS TOT

AU PLUS TARD

EN FONCTION DES EVENEMENTS

\section{SOCIO ECONOMLUES}

Au plus tot 1983

Au plus tard 1988

\section{COMMENTAIRES}

- Accélérateurs :

- Intégration européenne se développant au niveau des réseaux de communications.

- Diminution d'environ $10 \%$ du coût des transmissions de donnees.

- Coût de la main-d'oeuvre.

- Freins :

- Rejet de l'utilisation de l'informatique telle que developpée sur les bases actuelles

- Echecs dans les systèmes actuels d'éducation générale.

- Réglementations européennes concernant les constructeurs d'informatique non européennes. 


\begin{tabular}{|c|c|c|}
\hline \multicolumn{3}{|c|}{ ANALYSE DFE YURTRS PQBSCBLES } \\
\hline 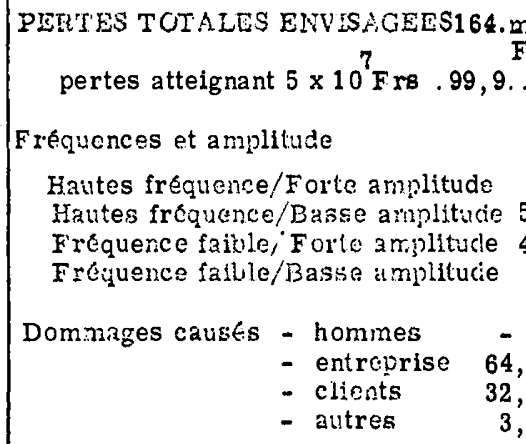 & $\begin{array}{l}\text { nillions } \\
\text { TS } \% \\
54,8 \\
45,2 \\
- \\
1 \\
8\end{array}$ & $\begin{array}{l}\text { Sunrces d'incidentis } \\
\text { - Equipements } \\
\text { - Logiciel } \\
\text { - Saisie } \\
\text { - Restitulion } \\
\text { - Erreurs utilisateurs } \\
\text { - Fraude - sabotage } \\
\text { - Divexs }\end{array}$ \\
\hline Principites sources d'incidents & $\begin{array}{l}\text { Tréquence } \\
\text { et amplitis- } \\
\text { de moyenne }\end{array}$ & $\begin{array}{l}\text { Prorosition de nusthodes } \\
\text { de reduction deis pertes }\end{array}$ \\
\hline $\begin{array}{l}\text { Le système général d'intercommu- } \\
\text { cations tombe en panne au moins } 3 \\
\text { heures d'où : } \\
\text { - arrêt des operations de transac- } \\
\text { tions } \\
\text { - pertes d'informations } \\
\text { coat de remise en oeuvre et de } \\
\text { correction. }\end{array}$ & $\begin{array}{c}4 / \text { an } \\
6 \underset{\text { millions }}{\text { FS }}\end{array}$ & $\begin{array}{l}\text { - Doubler le système général et cha- } \\
\text { cun de ses composants essentiels. } \\
\text { - Prévoir procédures manuelles ou } \\
\text { semi- automatiques. } \\
\text { - Assurance perte d'exploitation. }\end{array}$ \\
\hline $\begin{array}{l}\text { Pannes ou erreurs de manipulation } \\
\text { dans le fonctionnement des disposi- } \\
\text { tifs d'entrée terminaux dans le sys- } \\
\text { tème : } \\
\text { - erreurs dans les transactions } \\
\text { - coat de correction } \\
\text { - mẹcontement des clients et } \\
\text { dedommagement possibles }\end{array}$ & $\begin{array}{l}\text { 75. } 000 / \mathrm{an} \\
1200\end{array}$ & $\begin{array}{l}\text { - Systeme de controle et de secours. } \\
\text { - Formation des utilisateurs; } \\
\text { - Formalisation de procédures de } \\
\text { travail : documents-guides de pro- } \\
\text { cedure. }\end{array}$ \\
\hline $\begin{array}{l}\text { Sabotage ou fraude dans l'utilisation } \\
\text { du système, notamment en entrée } \\
\text { des infor mations et déboursements } \\
\text { de fonds peu importants mais pen- } \\
\text { dant une longue période. }\end{array}$ & $\begin{array}{c}5 / \text { an } \\
5,5 \text { millions } \\
\text { FS }\end{array}$ & $\begin{array}{l}\text { - Audit systématique des systèmes } \\
\text { d'informations par des conseils ex- } \\
\text { térieurs. } \\
\text { - Rotation du personnel. } \\
\text { - Systemes sophistiqués de controle } \\
\text { permanent du fonctionnement. }\end{array}$ \\
\hline $\begin{array}{l}\text { Sabotage par abandon volontaire } \\
\text { sans avertissement préalable de } \\
\text { l'exploitation des equipements infor- } \\
\text { matiques. } \\
\text { - cout de remise en oeuvre. }\end{array}$ & $\begin{array}{c}5 / \mathrm{an} \\
4,5 \mathrm{millions} \\
\text { FS }\end{array}$ & $\begin{array}{l}\text { - Renforcement des méthodes de choix } \\
\text { des operateurs. } \\
\text { - Automatisation complète des metho- } \\
\text { des d'exploitation. } \\
\text { - Politique plus participative dans la } \\
\text { gestion du personnel informatique. }\end{array}$ \\
\hline
\end{tabular}




\section{APPLICATION $\mathrm{N}^{2} 3$}

\section{GESTION PRODUCTION ASSEMBLAGE}

Pour $1 / 3$ des usines européennes d'assemblage, système central automatisé de planification et de suivi de toutes les phases d'assemblage.

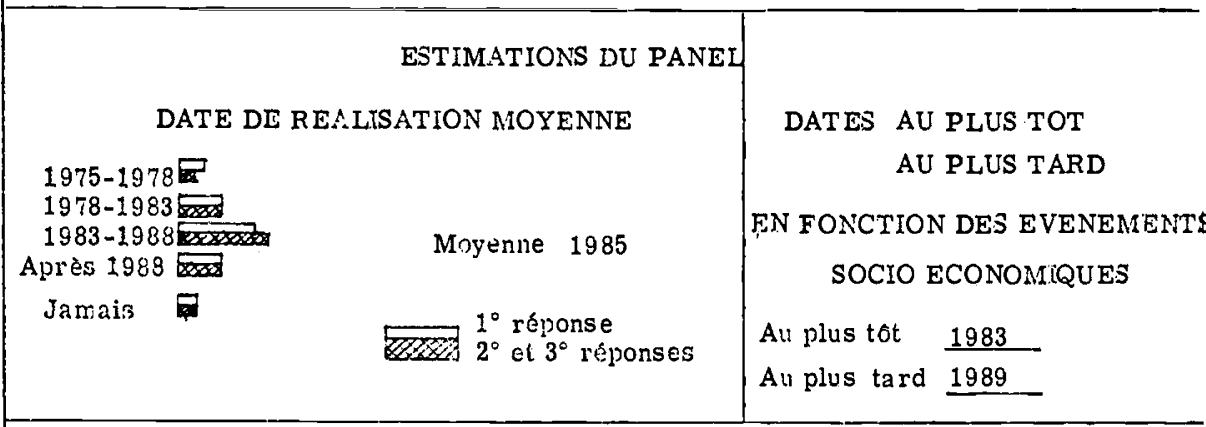

COMMENTAIRES

- Accélérateurs :

- Problèmes liés à la main d'oeuvre : coat et qualification.

- Concentration des entreprises dans ce secteur.

- Freins :

- Rejet du mode de travail actuel : organisation par chame.

- Stagnation économique ne favorisant pas les investissements nécessaires.

- Opposition des syndicats. 


\begin{tabular}{|c|c|c|}
\hline \multicolumn{3}{|c|}{ ANALYSE DES PER'SES POSSIBLES } \\
\hline 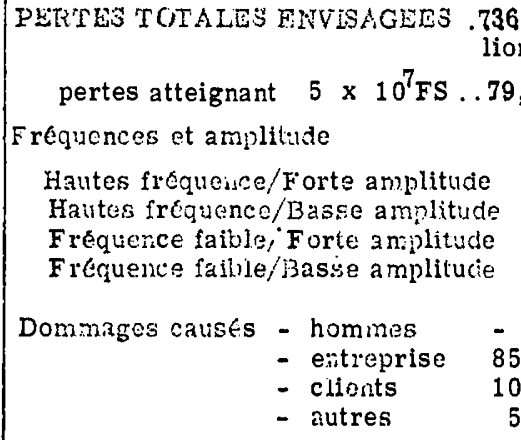 & $\begin{array}{l}, 5 \text { mil- } \\
\text { ns FS } \\
, 9 . \\
\\
\\
89,7 \\
6,7 \\
0,6\end{array}$ & 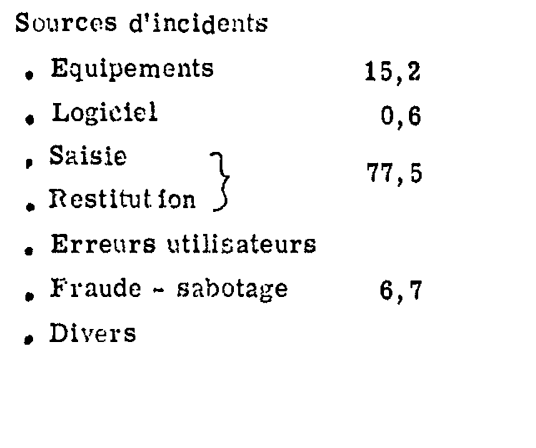 \\
\hline Principales soirces d'incidents & $\begin{array}{l}\text { Fréguence } \\
\text { et amplitis- } \\
\text { de moyenne } \\
\text { des incidents }\end{array}$ & $\begin{array}{l}\text { Propostition de methodes } \\
\text { de réduction des pertes }\end{array}$ \\
\hline $\begin{array}{l}\text { Le système tombe en panne plus } \\
\text { de } 3 \text { heures: } \\
\text { - perte de production } \\
\text { - cout de remise en oeuvre du } \\
\text { système et des chames d'as- } \\
\text { semblage } \\
\text { - perte des salaires. }\end{array}$ & $\begin{array}{l}1400 / \mathrm{an} \\
80.000 \mathrm{FS}\end{array}$ & $\begin{array}{l}\text { - Doublement des équipements cen- } \\
\text { traux. } \\
\text { - Procédures de dégradation douce. } \\
\text { - Formation intensive des utilisateurs } \\
\text { aux procédures de secours. } \\
\text { - Recours à l'informatique répartie }\end{array}$ \\
\hline $\begin{array}{l}\text { Matuvais fonctionnement et/ou arrêt } \\
\text { des équi pements de saisie et d'en- } \\
\text { trèe en ordinateur: } \\
\text { - perturbation des chames } \\
\text { - erreurs dans le produit final } \\
\text { - dédommagements aux clients }\end{array}$ & $\begin{array}{l}38.000 / \mathrm{an} \\
15.000 \mathrm{FS}\end{array}$ & $\begin{array}{l}\text { - Controle peimanent et précis du } \\
\text { système de saisie. } \\
\text { - Procédures développées de secours. }\end{array}$ \\
\hline $\begin{array}{l}\text { Sabotage volontaire à tous les ni- } \\
\text { veaux du système: } \\
\text { - perte de production } \\
\text { - erreurs dans le. produit final } \\
\text { - dédommagements aux clients }\end{array}$ & $\begin{array}{l}10 \text { par an } \\
450.000 \\
\text { FS }\end{array}$ & $\begin{array}{l}\text { - Développement des méthodes de } \\
\text { concertation et de participation du } \\
\text { personnel. } \\
\text { - Organisation en petites unités de } \\
\text { production. }\end{array}$ \\
\hline $\begin{array}{l}\text { Erreurs de logiciel } \\
\text { - perte de production } \\
\text { - cout de détection et de correc- } \\
\text { tion des erreurs } \\
\text { - mauvaise image de l'entreprise } \\
\text { chez ses clients. }\end{array}$ & $\begin{array}{l}500 / \mathrm{an} \\
100.000 \mathrm{FS}\end{array}$ & $\begin{array}{l}\text { - Essais extensifs et complets du } \\
\text { logiciel avant mise en oeuvre } \\
\text { - Développement de langages de pro- } \\
\text { grammation plus orientés vers } \\
\text { l'utilisateur. }\end{array}$ \\
\hline
\end{tabular}


APPLICATION No 4

SYSTEME BOURSIER

Cotation en temps réel des valeurs de bourse et traitement immédiat des transactions.

\section{ESTIMATIONS DU PANEL}

DATE DE REALISATION MOYENNE

1975-1978 品

1978-1983

1983-1988 자요

Après 1988

Moyenne 1982

Jamais

$1^{\circ}$ réponse

$2^{\circ}$ et $3^{\circ}$ réponses

DATES AU PLUS TOT

AU PLUS TARD

EN FONCTION DES EVENEMENSS

SOCIO ECONOMLQUES

Au plus tôt 1980

Au plus tard 1987

COMMENTAIFES

- Accélérations :

- Réglementation générale au niveau européen: intégration financière dans les 4 ou 5 grandes places boursières.

- Disponibilité de réseaux européens de communication, fiables et de coat moins élevé qu'actuellement.

- Accroissement de la clientèle actuelle par pénétration dans des couches socioprofessionnelles moins élevées.

- Freins :

- Non - intégration financière au niveau européen

- Réticences de la profession

- Continuation de la crise économique actuelle. 


\begin{tabular}{|c|c|c|}
\hline \multicolumn{3}{|c|}{ ANALYSE DES YER'SES POSSMBLES } \\
\hline 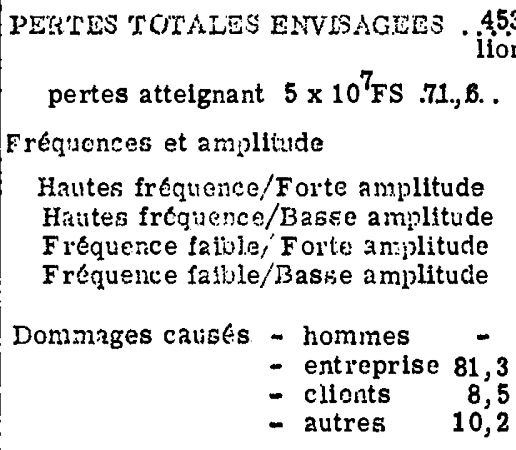 & $\begin{array}{l}\text { mil - } \\
\text { ins FS }\end{array}$ & $\begin{array}{lr}\text { Sources d'incidents } \\
\text { - Equipements } & 22,9 \\
\text { - Logiciel } & 55,2 \\
\text { - Saisie } & \\
\text { - Restitution } & \\
\text { - Erreurs utilisateurs } & 14,2 \\
\text { - Fraude - sabotage } & 7,7 \\
\text { - Divers } & \end{array}$ \\
\hline Prtncipales scurices d'iucidents & $\begin{array}{l}\text { Fréquonce } \\
\text { et amplitu- } \\
\text { de noyenne } \\
\text { des incidents }\end{array}$ & $\begin{array}{l}\text { Proposition de methodes } \\
\text { de réduction des pertes }\end{array}$ \\
\hline $\begin{array}{l}\text { Système en panne pendant plus de } \\
3 \text { heures, d'où : } \\
\text { - arrêt des transactions pour une } \\
\text { journée } \\
\text { - perte de revenus } \\
\text { - perte de clientèle } \\
\text { - dédommagements aux clients le- } \\
\text { sés. }\end{array}$ & $\begin{array}{c}80 / \text { an } \\
1,3 \text { millions } \\
\text { FS } \\
\end{array}$ & $\begin{array}{l}\text { - Doublement des unités vitales du sys- } \\
\text { tème. } \\
\text { Mise au point d'un système de fonc- } \\
\text { tionnement en dégradation douce. } \\
\text { - Création de procédures de secours } \\
\text { manuelles. } \\
\text { Assurance perte d'exploitation. }\end{array}$ \\
\hline $\begin{array}{l}\text { Anomalies de traitement dues au } \\
\text { logiciel du systeme conduisant à } \\
\text { - pertes d'informations } \\
\text { - mauvaises imputations } \\
\text { - pertes de clients }\end{array}$ & $\begin{array}{c}500 / \mathrm{an} \\
500.000 \mathrm{FS}\end{array}$ & $\begin{array}{l}\text { - Développement de langages spéciaux } \\
\text { détectant les erreurs de conception. } \\
\text { Essais du nouveau système en paral- } \\
\text { lele avec l'ancien système. } \\
\text { Tests de simulation des conditions } \\
\text { de fonctionnement. }\end{array}$ \\
\hline $\begin{array}{l}\text { Sabotage du système total d'où : } \\
\text { - arrêt des transactions pour } \\
\text { des durées de } 2 \text { à } 3 \text { jours } \\
\text { - pertes de revenus } \\
\text { - pertes de clientèle et dédom- } \\
\text { magement des clients lésés. } \\
\text { - cout de remise en oeuvre. }\end{array}$ & $\begin{array}{l}10 \text { par anl } \\
3,5 \text { millions } \\
\text { par an }\end{array}$ & $\begin{array}{l}\text { - Création de procédures de secours } \\
\text { manuelles. } \\
\text { - Dśveloppement d'un système auto- } \\
\text { nome de détection des fraudes et sa- } \\
\text { botage. } \\
\text { Renforcement des méthodes de choix } \\
\text { des informaticiens. }\end{array}$ \\
\hline $\begin{array}{l}\text { Ent rée incorrecte, par l'utilisateur, } \\
\text { des informations nécessaires all } \\
\text { système d'où : } \\
\text { - perturbation dans les transac- } \\
\text { tions } \\
\text { coơt de correction }\end{array}$ & $1600 / \mathrm{an}$ & \begin{tabular}{|l|} 
- Formation poussée des utilisateurs. \\
- Prișe en charge de chaque informa- \\
tion par deux agents. \\
- Utilisation de moyens automatiques \\
permettant de réduire le nombre d'er \\
reurs (badges, cartes d'identification) \\
contróle par retour immédiat de la \\
transaction.
\end{tabular} \\
\hline
\end{tabular}


APPLICATION No 7

SYSTEME DE MARKETING

$\mathrm{Au}$ moins 100 grandes entreprises industrielles en Europe auront en fonctionnement un système complet en temps réel de marketing comprenant une base de données avec toutes les informations pour : études prévisionnelles, administration des ventes, statistiques commerciales, controle des couts de distribution et études de stratégie commerciale (segmentation du marché, par exemple).

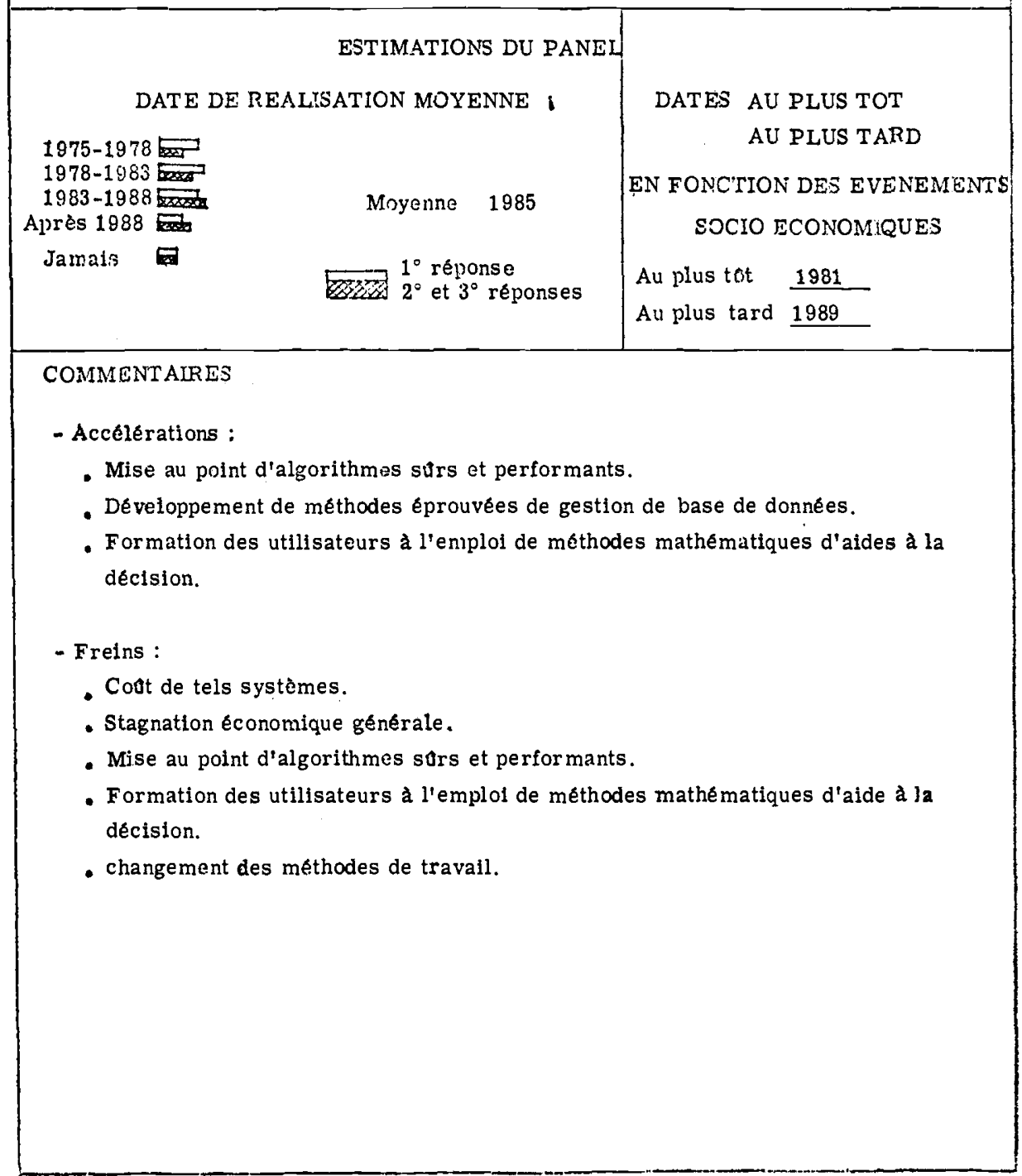




\begin{tabular}{|c|c|c|}
\hline \multicolumn{3}{|c|}{ ANALYSE DES PERTES PGSBBLES } \\
\hline 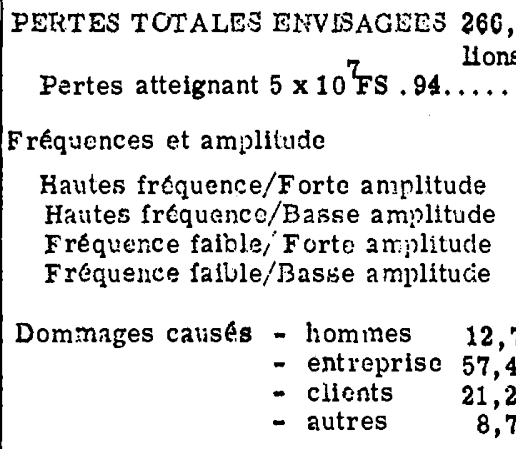 & $\begin{array}{l}\text {, } 9 . \mathrm{mil}- \\
\text { is FS. } \\
\% \\
7 \\
4 \\
2 \\
7\end{array}$ & $\begin{array}{l}\text { Suurces d'incidents } \\
\text { - Equipenients } \\
\text { - Logiciel } \quad 34,2 \\
\text { - Srisie } \quad 59,9 \\
\text { - Restitution } \\
\text { - Erreurs utilisateurs } 3,9 \\
\text { - Fraude - sabotage } \\
\text { - Divers (exploitation } 2 \%\end{array}$ \\
\hline Principiles solirces d'incidents & $\begin{array}{l}\text { Ir réguonce } \\
\text { et. amplstu- } \\
\text { de moyenue } \\
\text { des incidents }\end{array}$ & $\begin{array}{l}\text { Proposition de minthodes } \\
\text { de reduction des pertes }\end{array}$ \\
\hline $\begin{array}{l}\text { Erreurs de conception dans les al- } \\
\text { gorithmes de préparation des déci- } \\
\text { sions d'oú : } \\
\text { - pertes d'exploitation } \\
\text { - perte de clientèle } \\
\text { - détection et correction des } \\
\text { erreurs. }\end{array}$ & $\begin{array}{l}130 \text { par an } \\
700.000 \mathrm{FS}\end{array}$ & $\begin{array}{l}\text { - Developpement de méthodes préci - } \\
\text { ses d'essais du nouveau système. } \\
\text { - Fonctionnement en parallèle du nou- } \\
\text { veau système avec l'ancienne mé- } \\
\text { thode. } \\
\text { - Mise en route progressive des dif- } \\
\text { férentes fonctions du système. }\end{array}$ \\
\hline $\begin{array}{l}\text { Alté ration des informations entrées } \\
\text { dans le système conduisant a : } \\
\text { - détection et correction des } \\
\text { erreurs. } \\
\text { - retards dans l'utilisation du } \\
\text { système. }\end{array}$ & $\begin{array}{l}20.000 / \mathrm{an} \\
8.000 / \mathrm{an}\end{array}$ & $\begin{array}{l}\text { - Système de controle des information } \\
\text { entrées dans le système. } \\
\text { - Audit permanent et systématique des } \\
\text { nisthodes de saisie. } \\
\text { - Système de sauvegarde des informa } \\
\text { tions en entrée. }\end{array}$ \\
\hline $\begin{array}{l}\text { Mauvaise utilisation des résultats } \\
\text { issus du système d'où : } \\
\text { - mauvaises orientations straté- } \\
\text { giques } \\
\text { - pertes de certains marchés } \\
\text { - insatisfaction des utilisateurs. }\end{array}$ & \begin{tabular}{|c|}
$300 / \mathrm{an}$ \\
$350.000 \mathrm{FS}$
\end{tabular} & $\begin{array}{l}\text { - Formation intensive des utilisateurs } \\
\text { - Procédure précises d'utilisation des } \\
\text { résultats. } \\
\text { - Sinulation simplifiée sur les don- } \\
\text { nées critiques. }\end{array}$ \\
\hline $\begin{array}{l}\text { Erreur de manipulation dans l'uti- } \\
\text { lisation du logiciel : utilisation de } \\
\text { programmes antérieurs à certaines } \\
\text { modifications conduisant a : } \\
\text { - travaux a refaire } \\
\text { - perte de temps }\end{array}$ & $\begin{array}{l}45 \text { par an } \\
120.000 \mathrm{FS}\end{array}$ & $\begin{array}{l}\text { - Organisation rigoureuse de l'exploi- } \\
\text { tation du nouveau système. } \\
\text { - Formation des opérateurs informa- } \\
\text { tíciens. } \\
\text { - Developpement par les construc- } \\
\text { teurs de systèmes opératoires axés } \\
\text { sur la sécurité des traitements. }\end{array}$ \\
\hline
\end{tabular}


Au moins $30 \%$ des pools dactylographiques utilisant plus de trois personnes dans les entreprises européennes, seront dotés de systèmes de traitement de textes pour augmenter la productivité et rendre le travail plus agréable.

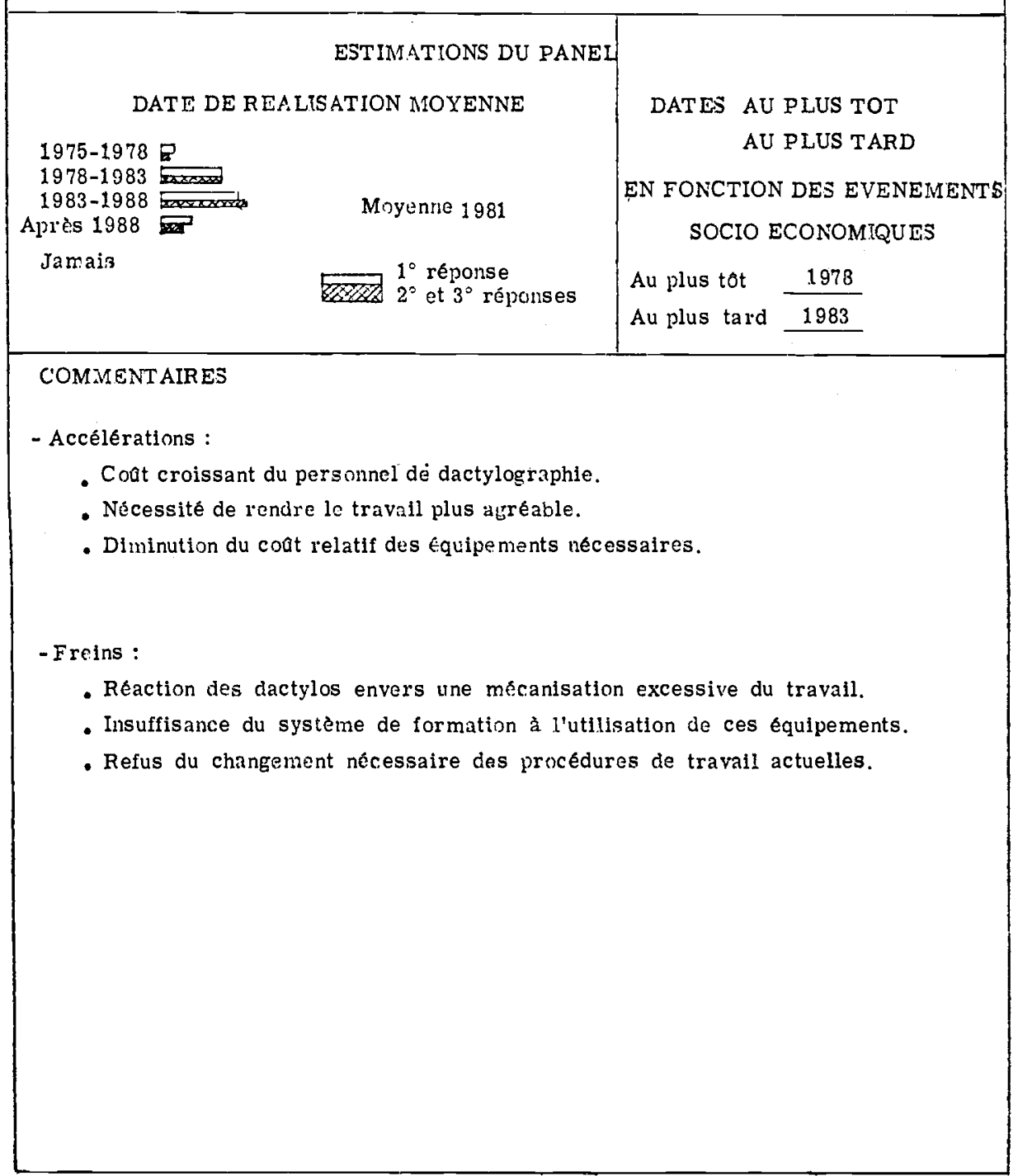




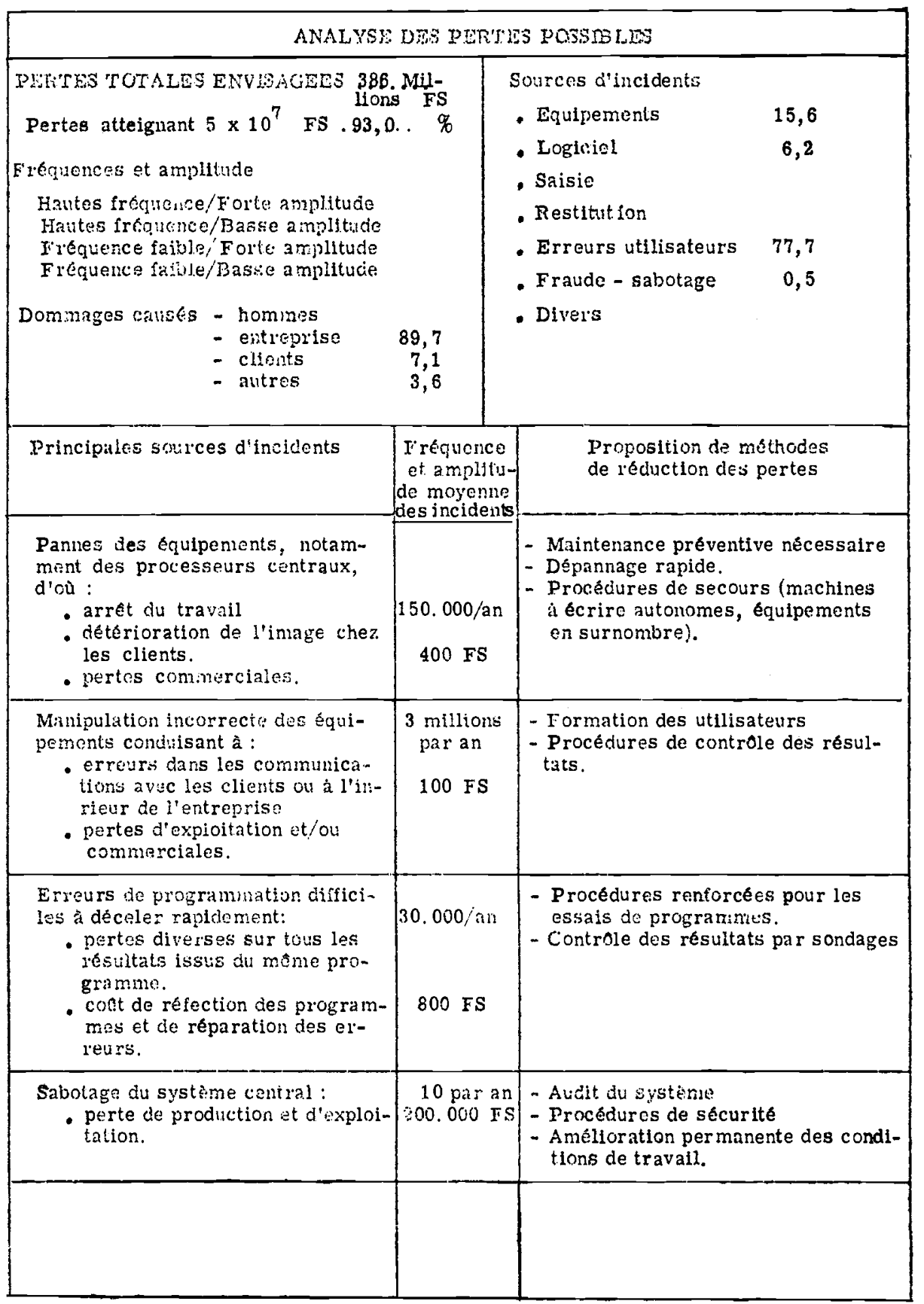




\section{APPLICATION $N^{\circ} 11$ GESTION DE LA DISTRIBUTION}

Au moins $75 \%$ de toutes les chames de distribution avec plus de 10 magasins en Europe auront un système de gestion intégrée comprenant la salsie des ventes en temps réel, la gestion des stocks, le réapprovisionnement et la gestion des fournisseurs pour tous les articles non périssables.

\section{ESTIMATIONS DU PANEL \\ DATE DE REALISATION MOYENINE}

DATES AU PLUS TOT

1975-1978

$1978-1983$

$1983-1088$ क्ति

Après 1988 是

Moyenne 1984

Jamais

$1^{\circ}$ repponse

$2^{\circ}$ et $3^{\circ}$ réponses
AU PLUS TARD

EN FONCTION DES EVENEMENTS

SOCIO ECONOMQUES

Au plus tôt 1981

Au plus tard 1984

\section{COMMENTAIRES}

-Accélérations :

- Concentration du commerco de distribution.

- Limitation par reglcmentation des marges de distribution d'oà un besoin accru de mieux connaftre et controler les vertes.

- Reduction du tomps de travall pour les enployes du commerce et autgmentation des heures d'ouverture des magasins.

- Freins :

- Aggravation de la récession économiaue.

- Arret de la tendance à la concentration du commerce de distribution.

- Rejet du travail fastidieux, répètitif, 


\begin{tabular}{|c|c|c|}
\hline \multicolumn{3}{|c|}{ ANALYSE DES PERTES JOSSIBLES } \\
\hline $\begin{array}{l}\text { Fréquences et amplitude } \\
\text { Hautes fréquence/Forte anplitude } \\
\text { Hautes fréquence/Baase amplitude } \\
\text { Fréquence faible/ Forte amplitude } \\
\text { Fréquence faible/Basse amplitucie } \\
\begin{aligned} & \text { Dommages causes } \text { - hommes } \\
& \text { - entreprise } 89 \\
& \text { - clients } 13 \\
& \text { - autres }\end{aligned}\end{array}$ & $\begin{array}{l}, 6 \\
, 3 \\
, 1\end{array}$ & 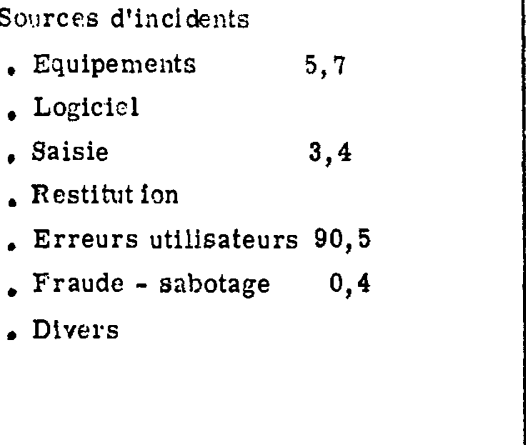 \\
\hline Principales sources d'incidents & $\begin{array}{l}\text { Frequence } \\
\text { et amplitu- } \\
\text { de moyenne } \\
\text { des incidents }\end{array}$ & $\begin{array}{l}\text { Proposition de methodes } \\
\text { de réduction dés pertes }\end{array}$ \\
\hline $\begin{array}{l}\text { Le système principal tombe en pan- } \\
\text { ne au molns trois heures d'oú : } \\
\text { - ralentissement des ventes } \\
\text { - mécontentement de la clientèle } \\
\text { - rupture du rythme de réappro- } \\
\text { visionnement. }\end{array}$ & $\begin{array}{l}200 \mathrm{par} \text { an } \\
200.000 \mathrm{FS}\end{array}$ & $\begin{array}{l}\text { - Doublement des éléments critiques } \\
\text { du système } \\
\text { - Utilisation de l'lnformatique répar- } \\
\text { tie (plusieurs processeurs par } \\
\text { système) } \\
\text { - Procedures manuelles ou semi- } \\
\text { manuelles de secours. }\end{array}$ \\
\hline $\begin{array}{l}\text { Mauvais fonctionneirient des équi- } \\
\text { pements de saisie conduisant à : } \\
\text { - erreurs de facturation et/ou de } \\
\text { commande. } \\
\text { - diminution du service clientèle } \\
\text { - cout de remise en ordre. }\end{array}$ & $\begin{array}{c}2000 \text { par an } \\
12.000 / \text { an }\end{array}$ & $\begin{array}{l}\text { - Maintenance préventive et systéma- } \\
\text { tique. } \\
\text { - Logiclel assurant en permanence } \\
\text { les tests de vraisemblance des équi- } \\
\text { ments de saisie. } \\
\text { - Utilisation de procédures de "feed- } \\
\text { back" permettant l'affichage sur } \\
\text { ecrans des informations entrées dans } \\
\text { l'ordinateur central. }\end{array}$ \\
\hline $\begin{array}{l}\text { Erreur dans l'enregistrement des } \\
\text { ventes d'où: } \\
\text { - perte de clientèle (soit par ven- } \\
\text { tes manqués par non réapprovi } \\
\text { sionnement soit par cause d'er- } \\
\text { reurs contre la clientèle). } \\
\text { - pertes directes. }\end{array}$ & $\mid \begin{array}{l}2,5 \text { millions } \\
\text { par an } \\
260 \mathrm{FS}\end{array}$ & $\begin{array}{l}\text { - Education du personnel. } \\
\text { - Controle sophistiqué, par logiciel } \\
\text { ou par hardware, du travail de sai- } \\
\text { sie. } \\
\text { - Mise au point d'un rythme de travail } \\
\text { de nature différente ; ex : } 1 / 2 \text { heure } \\
\text { saisie ; } 1 / 2 \text { heure vente. }\end{array}$ \\
\hline $\begin{array}{l}\text { Fraude lors de la saisie des infor- } \\
\text { mations notamment aux points de } \\
\text { ventes conduisant à des pertes im- } \\
\text { portantes occasionnées principale- } \\
\text { ment par des bandes organisées. }\end{array}$ & $\begin{array}{c}10 \text { par an } \\
300.000 \mathrm{FS}\end{array}$ & $\begin{array}{l}\text { - Audit permanent du système. } \\
\text { - Logiciel spécifique permettant le } \\
\text { controle aléatoire par sondages } \\
\text { répétitifs. } \\
\text { - Education du personnel. } \\
\text { - Rotation du personnel entre les dif- } \\
\text { férents postes de travail. }\end{array}$ \\
\hline
\end{tabular}




\section{APPLICATION N $N^{\circ} 13$ CONTROLE DE PROCESSUS DANS LES RAFFINERIES}

Toutes les raffineries de pétrole installées en Europe fonctionneront complètement sous le controle d'un système automatique.

\section{ESTIMATIONS DU PANEL \\ DATE DE REALISATION MOYENNE}

$1975-1978$

$1978-1983$

$1983-1988$

Après 1988

Jamais
Moyenne 1981

$1^{\circ}$ réponse

$2^{\circ}$ et $3^{\circ}$ réponses

\section{DATES AU PLUS TOT} AU PLUS TARD

EN FONCTION DES EVENEMENTS SOCIO ECONOMIQUES

Au plus tot 1979

Au plus tard 1985

\section{COMMENTAIRES}

Dans un domaine où les réalisations de ce type ont commencé voici déjâ dix ans, on peuf souligner :

- Accélérations :

- Augmentation croissante du coat du pétrole brut d'ou la nécessité de maximiser le rendement des raffineries.

- Construction de raffineries avec une capacité de production croissante.

- Besoins de mieux maftriser la pollution.

\section{- Freins :}

- Ralentissement de la consommation des produits pétroliers.

- Problèmes de pollution et d'environnement (qui peuvent freiner la construction de nouvelle raffineries).

- Coot de la mise en place du système dans les petites et anciennes raffineries. 


\begin{tabular}{|c|c|c|}
\hline \multicolumn{3}{|c|}{ ANALYSE DES YERTES YCSSDBLES } \\
\hline 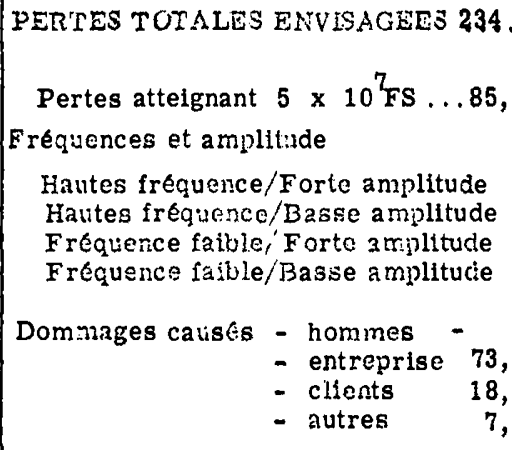 & $\begin{array}{l}\text { millions } \\
4 \% \% \\
14,6 \\
85,4 \\
4 \\
7 \\
9\end{array}$ & $\begin{array}{l}\text { Sources d'incidents } \\
\text { - Equipements } \\
\text { - Logiciel } \\
\text { - Saisie } \\
\text { - Restitution } \\
\text { - Erreurs utilisateurs } \\
\text { - Fraude - sabotage } \quad 4,2 \\
\text { - Divers }\end{array}$ \\
\hline Principales sources d'incidents & $\begin{array}{l}\text { Fréquence } \\
\text { et amplitu- } \\
\text { de moyenne } \\
\text { des incidents }\end{array}$ & $\begin{array}{l}\text { Proposition de mithodes } \\
\text { de reduction des pertes }\end{array}$ \\
\hline $\begin{array}{l}\text { Le système tombe en panne au } \\
\text { moins une heure d'ou : } \\
\text { - pertes de production } \\
\text { - pertes de revenus. }\end{array}$ & $\begin{array}{l}6 \text { par an } \\
2 \text { millions } \\
\text { FS }\end{array}$ & $\begin{array}{l}\text { - Doublement du système ou } \\
\text { Procédures de sauvegarde. } \\
\text { - Assurance de perte d'exploitation. } \\
\text { - Utilisation de systèmes interconnec- } \\
\text { tés d'infor matique répartie. }\end{array}$ \\
\hline $\begin{array}{l}\text { Erreurs de programmation aboutis- } \\
\text { sant a : } \\
\text { - production mal ajustée aux be- } \\
\text { soins } \\
\text { - pertes en rendements } \\
\text { - non satisfaction des clients. }\end{array}$ & $\left|\begin{array}{c}10 \text { par an } \\
1,2 \text { millions }\end{array}\right|$ & $\begin{array}{l}\text { - Essais rigoureux des programmes } \\
\text { et simulation des résultats. } \\
\text { - Formation plus complète des infor- } \\
\text { ticiens aux problèmes de régulation } \\
\text { des processus. }\end{array}$ \\
\hline $\begin{array}{l}\text { Non fonctionnement ou fonctionne- } \\
\text { ment perturbé des equipements de } \\
\text { commande des processus } \\
\text { - retards en production } \\
\text { - coot de remplacement }\end{array}$ & $\left|\begin{array}{l}5000 \text { par an } \\
40.000 \mathrm{FS}\end{array}\right|$ & $\begin{array}{l}\text { - Doublement des équipements de com } \\
\text { mande de processus, ou procédures } \\
\text { - de sauvegarde. } \\
\text { - Assurance pertes d'exploitation } \\
\text { - Maintenance préventive des équipe- } \\
\text { ments de commande }\end{array}$ \\
\hline $\begin{array}{l}\text { Sabotage des installations de con- } \\
\text { trôle de processus : } \\
\text { - pertes de production } \\
\text { - pertes de revenus } \\
\text { - coat de reconstitution du sys- } \\
\text { tème. }\end{array}$ & $\begin{array}{l}2 \text { par an } \\
5 \text { millions } \\
\text { FS }\end{array}$ & $\begin{array}{l}\text { - Système rigoureux de sécurité pour } \\
\text { les équipements. } \\
\text { - Procédures de sauvegarde. }\end{array}$ \\
\hline
\end{tabular}


APPLICATION No 15 CONTROLE DE FABRICATION DANS L'INDUSTRIE ALIMENTAIRE.

Plus de $25 \%$ des entreprises européennes de l'industrie alimentaire utiliseront un controle automatisé des processus de fabrication pour pi loter et surveiller la qualité de la production et notamment la pureté des produits fabriqués, et ceci pour au moins la moitié de leurs produits.

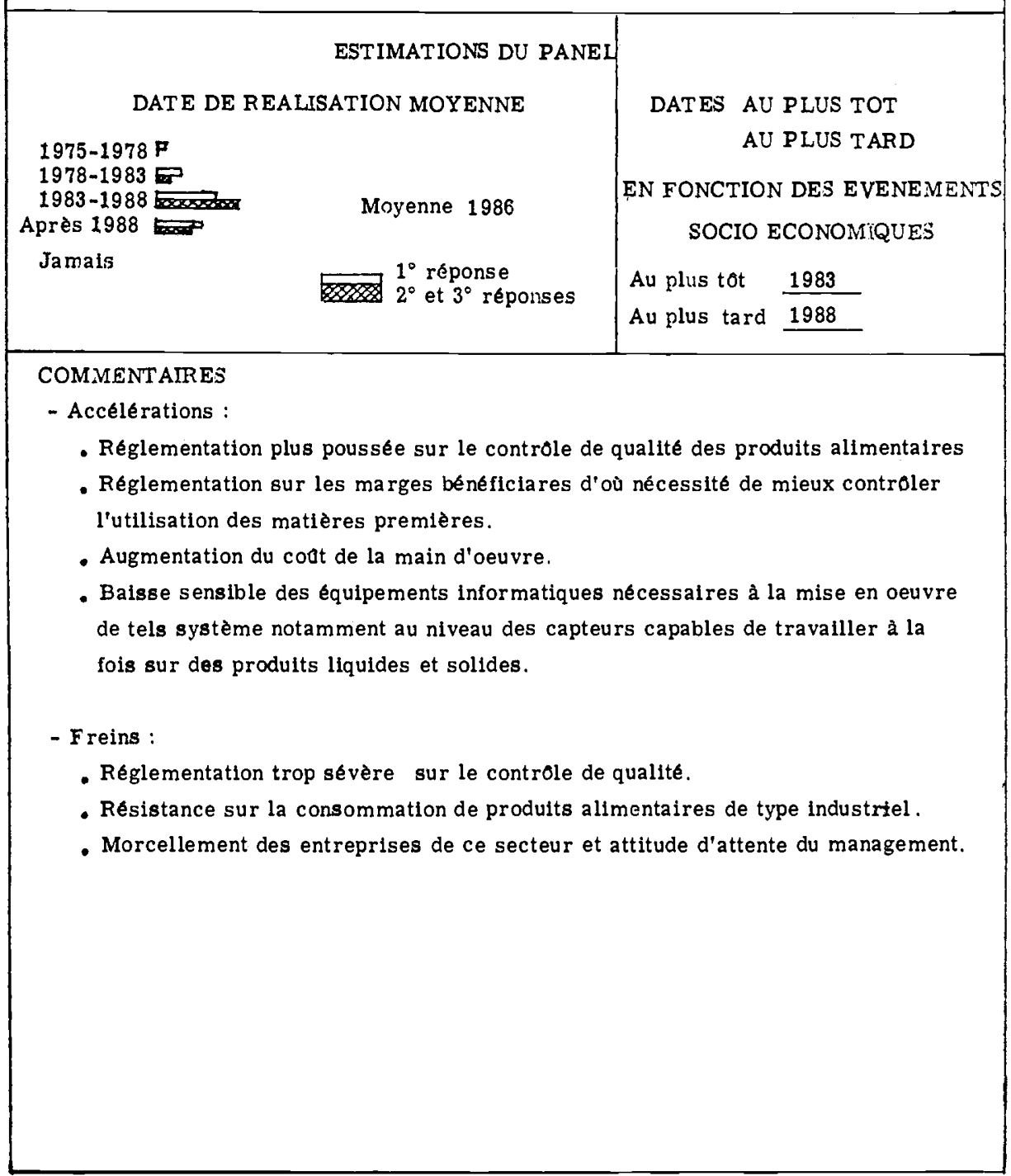




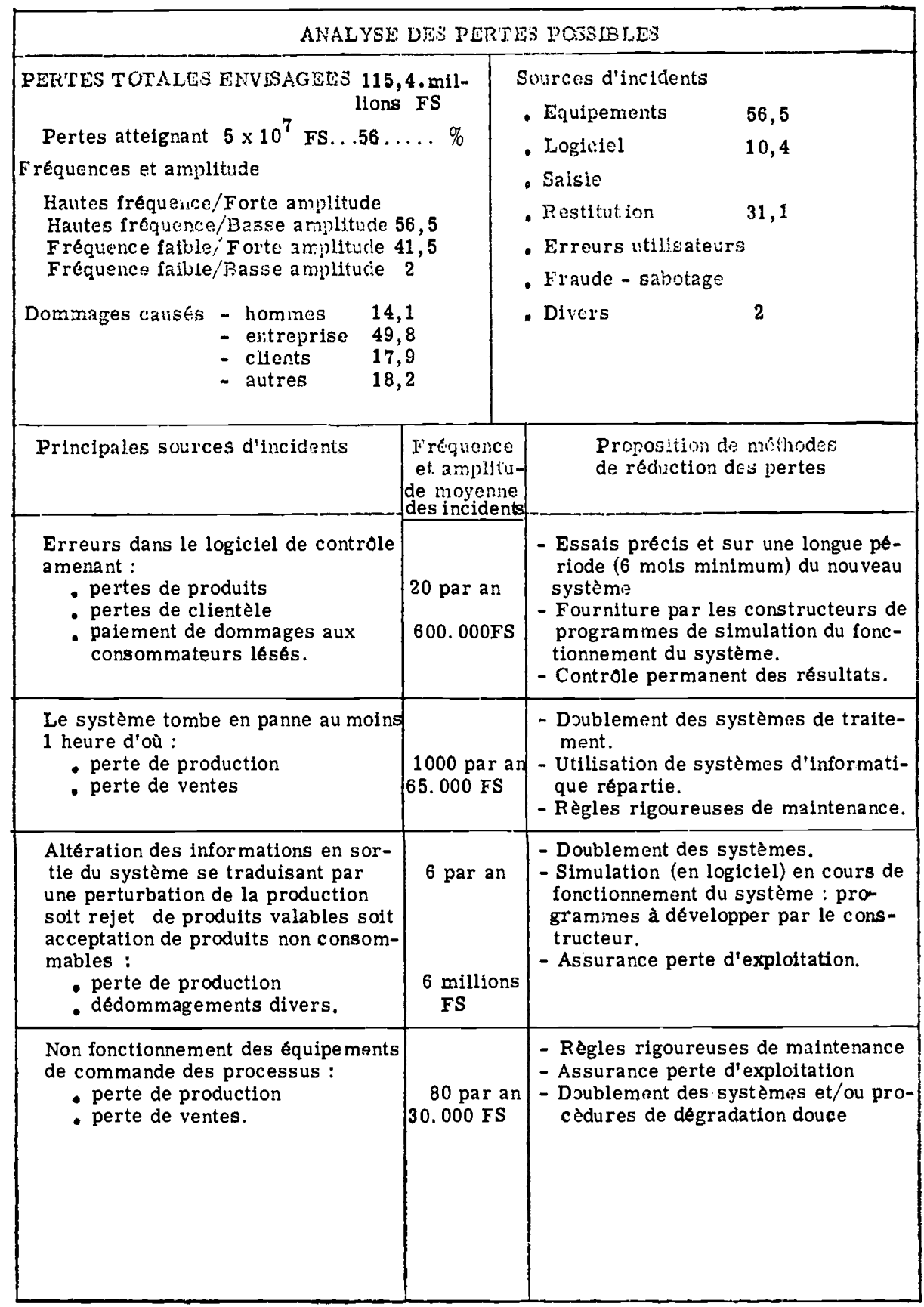




\section{APPLICATION N $N^{2} 17$ CONTROLE DE FABRICATION DANS L'INDUSTRIE}

PHAR MACEUTIQUE.

Plus de $50 \%$ des entreprises européennes de l'industrie pharmaceutique utiliseront pour tous leurs produits un système automatisé intégré pour controler et surveiller la qualité de la production.

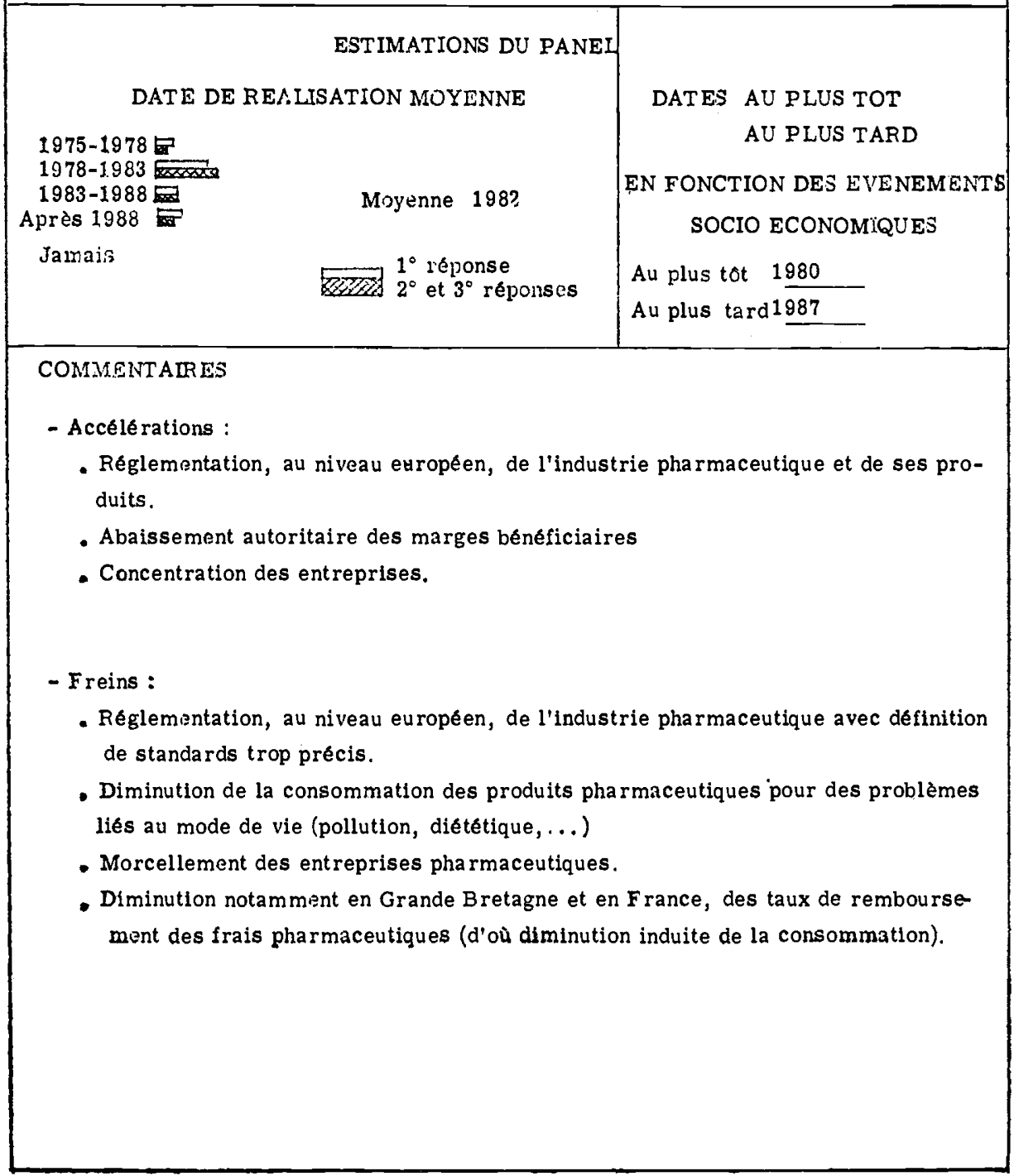




\begin{tabular}{|c|c|c|}
\hline \multicolumn{3}{|c|}{ ANAISYSL DES PERIES POSSCBLES } \\
\hline 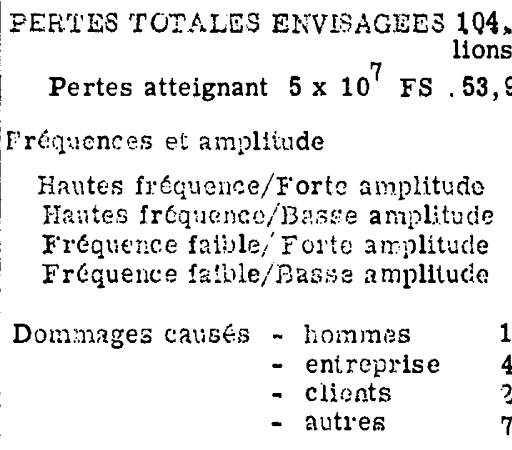 & $\begin{array}{l}2, \mathrm{mil}- \\
\text { FS } \\
9 \ldots \\
\\
85,5 \\
14,5 \\
19,7 \\
44,4 \\
28,6 \\
7,3\end{array}$ & $\begin{array}{l}\text { Sources d'incid ents } \\
\begin{array}{l}\text { - Equivements } \\
\text { - Logiciel }\end{array} \\
\text { - Saisie } \\
\text { - Restitution } \\
\text { - Erreurs utilisateurs } 26,9 \\
\text { - Fraude - sahotage } \\
\text { - Divers }\end{array}$ \\
\hline Primipales sorirces d'inctients & $\begin{array}{l}\text { Fréguence } \\
\text { et amplitu- } \\
\text { de moyenne } \\
\text { des incidents }\end{array}$ & $\begin{array}{l}\text { Proposition de mithodes } \\
\text { de réduction des pertes }\end{array}$ \\
\hline $\begin{array}{l}\text { Fausses (ou incomplètes) informa- } \\
\text { tions fournies au système notam- } \\
\text { ment dans les processus de la fabri- } \\
\text { cation (gammes, nomenclatures) d'où } \\
\text { - pertes de productions } \\
\text { - pertes de clientèle }\end{array}$ & $\begin{array}{l}4 \text { par an } \\
7 \text { millions } \\
\text { FS }\end{array}$ & $\begin{array}{l}\text { - Controle rigoureux des données } \\
\text { avant l'entrée dans le système. } \\
\text { - Controle des données manquantes. } \\
\text { - Simulation des résultats obtenus } \\
\text { pendant au moins un an. } \\
\text { - Essais du système en parallèle avec } \\
\text { ancien système pendant un an. }\end{array}$ \\
\hline $\begin{array}{l}\text { Erreur de conception dans le logi- } \\
\text { ciel de controle et commande ame- } \\
\text { nant : } \\
\text { - pertes de production } \\
\text { - pertes de clientèle } \\
\text { - dédommagement des clients } \\
\text { lésés. }\end{array}$ & $\begin{array}{l}2 \text { par an } \\
28 \text { millions } \\
\text { FS }\end{array}$ & $\begin{array}{l}\text { - Essais sur une période d'au moins } \\
\text { un an du nouveau système en pa- } \\
\text { rallèle avec l'ancien. } \\
\text { - Mise au point de méthodes de con- } \\
\text { trole permanent des résultats } \\
\text { (audit technique systématique). }\end{array}$ \\
\hline $\begin{array}{l}\text { Non fonctionnement des analyseurs } \\
\text { d'où : } \\
\text { - perte de production } \\
\text { - perte de matières premières } \\
\text { - pertes de ventes. }\end{array}$ & $\begin{array}{l}60 \text { par an } \\
86.000 \mathrm{FS}\end{array}$ & $\begin{array}{l}\text { - Assurance responsabilité civile et } \\
\text { perte d'exploitation. } \\
\text { - Règles rigoureuses de maintenance } \\
\text { préventive. } \\
\text { - Doublement des dispositifs et/ou } \\
\text { mise en oeuvre de procédure de } \\
\text { dégration douce. } \\
\text { - Programme de tests des analyseurs } \\
\text { inclus dans le système géné ral. }\end{array}$ \\
\hline $\begin{array}{l}\text { Altération des informations en sor- } \\
\text { tie du système se traduisant par une } \\
\text { pertubation de la production: } \\
\text { - pertes de produits valables } \\
\text { - dédommagements divers. }\end{array}$ & $\begin{array}{l}1 / 2 \text { par an } \\
10 \text { millions }\end{array}$ & $\begin{array}{l}\text { - Assurance responsabilité civile et } \\
\text { perte d'exploitation. } \\
\text { - Doublement des dispositifs de res- } \\
\text { titution. } \\
\text { - Tests de fonctionnement inclus dans } \\
\text { le système général. }\end{array}$ \\
\hline
\end{tabular}




\section{APPLICATION No 18 CONTROLE DE FABRICATION DANS L'INDUSTRIE CHIMIQUE}

Au moins $65 \%$ des usines européennes de l'industrie chimique utiliseront un système automatisé intégré pour contrôler les processus de fabrication et surveiller la qualité de la production.

\section{ESTIMATIONS DU PANEL}

DATE DE REALISATION MOYENNE

$1975-1978$

$1978-1983$

$1983-1988$

Après 1988

Jamais
Moyenne 1983

$1^{\circ}$ réponse

$2^{\circ}$ et $3^{\circ}$ reponses
DATES AU PLUS TOT

$A U$ PLUS TARD

EN FONCTION DES EVENEMENTS

SOCIO ECONOM:QUES

Au plus tôt 1981

Au plus tard 1990

\section{COMMENT AIRES}

Les expériences en cours permettent de déterminer les facteurs qui peuvent influer sur la date de réalisation moyenne prévue :

- Accélérations :

- Augmentation importante du coút des matières premières.

- Millleure utilisation des capacités de production actuelles.

- Augmentation de la main d'oeuvre et répugnance de celle-ci à accomplir des travaux pénibles et insalubres.

- Réglementation internationale anti-pollution.

- Freins :

- Aggravation de la présente crise économique.

- Cout de la mise en place d'un système de ce type dans les unités de production déjà vieilles

- Réglementation sur la production et la consommation de produits polluants et/ou dangereux pour l'environnement. 


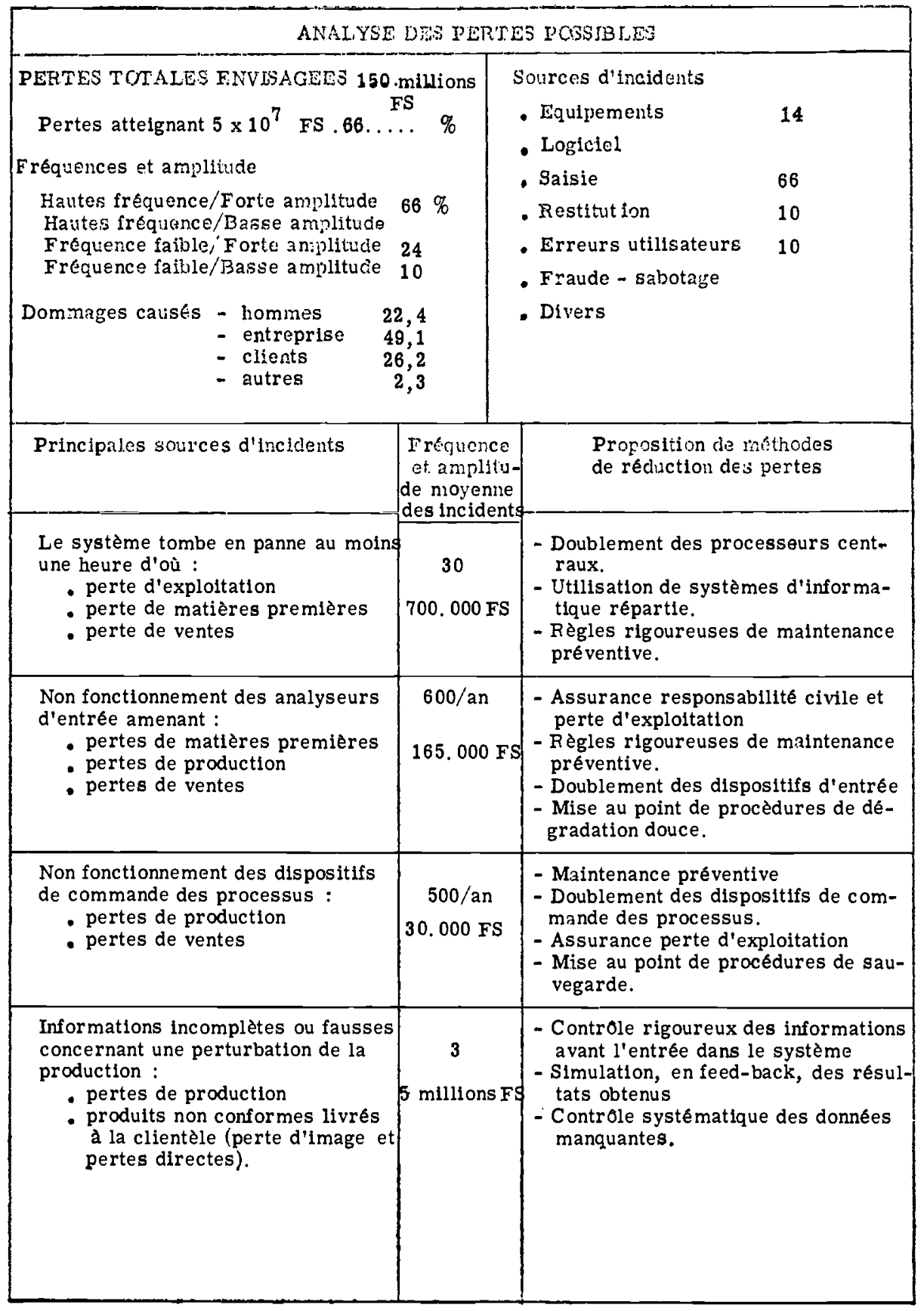


A]PPIICATION N 19 CONTROLE DE FABRICATION DANS L'INDUSTRIE DU PNEU

Plus de $75 \%$ des usines de fabrication de pneumatiques en Europe utiliseront un système complet de controle des processus de fabrication

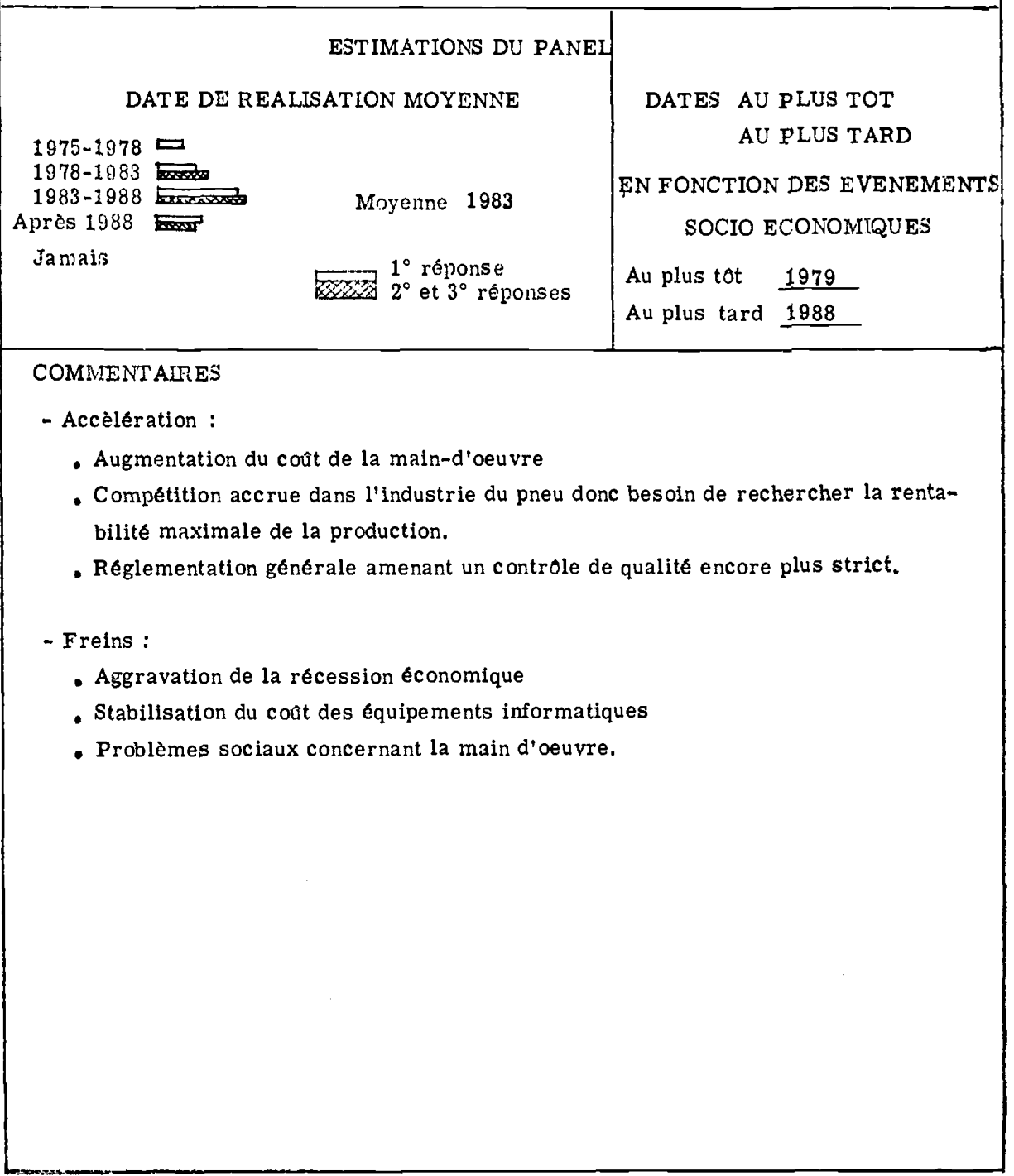




\begin{tabular}{|c|c|c|}
\hline \multicolumn{3}{|c|}{ ANALYSE DSE PERTES POSSDBLES } \\
\hline 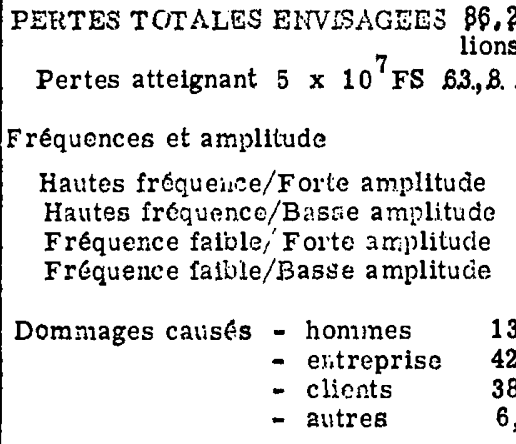 & $\begin{array}{l}\text { mil- } \\
\ldots 8 \\
8, \ldots \\
8,4,6 \\
3,5 \\
8,2 \\
, 3\end{array}$ & $\begin{array}{l}\text { Sources d'incidents } \\
\text { - Equipements } \\
\text { - Logiciel } \\
\text { - Saisie } \\
\text { - Restitut ion } \\
\text { - Erreurs utilisateurs } \\
\text { - Fraude - sabotage } \\
\text { - Divers }\end{array}$ \\
\hline Principales sources d'incidents & $\begin{array}{l}\text { Frequence } \\
\text { et amplitu- } \\
\text { de moyenne } \\
\text { des incidents }\end{array}$ & $\begin{array}{l}\text { Proposition de methodes } \\
\text { de leduction des pertes }\end{array}$ \\
\hline $\begin{array}{l}\text { Le systeme tombe en panne au moins } \\
\text { une heure : } \\
\text { - perte de production } \\
\text { - perte de ventes } \\
\text { - perte de salaire } \\
\text { - perte de matières premieres. }\end{array}$ & $\begin{array}{c}30 / \mathrm{an} \\
240.000 \mathrm{FS}\end{array}$ & $\begin{array}{l}\text { - Doublement des éléments critiques } \\
\text { du système: } \\
\text { - et/ou Procèdures de dégradation } \\
\text { douce } \\
\text { - Utilisation de l'informatique répar- } \\
\text { tie. } \\
\text { - Assurance perte d'exploitation. }\end{array}$ \\
\hline $\begin{array}{l}\text { Non fonctionnement des equipements } \\
\text { de commande des processus condui- } \\
\text { sant a : } \\
\text { - perte de production et de ventes } \\
\text { - perte de matieres premières. }\end{array}$ & $\begin{array}{l}500, \mathrm{an} \\
16.000 \mathrm{FS}\end{array}$ & $\begin{array}{l}\text { - Maintenance préventive systémati- } \\
\text { que, avec simulation de fonction- } \\
\text { nement, des dispositifs de commande } \\
\text { - Doublement des éléments critiques. }\end{array}$ \\
\hline $\begin{array}{l}\text { Non fonctionnement des équipements } \\
\text { de saisie des informations; } \\
\text { - perte de production et de vente } \\
\text { - perte de matières premières. }\end{array}$ & $\begin{array}{l}5000 / \mathrm{an} \\
11.000 \mathrm{FS}\end{array}$ & $\begin{array}{l}\text { - Doublement des équipements de sai- } \\
\text { sie. } \\
\text { - Conception de dispositifs à haute } \\
\text { fiabilité. } \\
\text { - Logiciel de tests permanents du sys- } \\
\text { tème de saisie. }\end{array}$ \\
\hline $\begin{array}{l}\text { Fonctionnement perturbé dans les } \\
\text { équipements de saisie des informa- } \\
\text { tions : } \\
\text { - produits non conformes aux spé } \\
\text { cifications d'où dédommage- } \\
\text { ments éventuels aux clients. } \\
\text { - perte de production. }\end{array}$ & $\begin{array}{l}500 / \mathrm{an} \\
32.000 \mathrm{FS}\end{array}$ & $\begin{array}{l}\text { - Logicjel de tests permanents du sys- } \\
\text { tème de saisie. } \\
\text { - Procèdures de dégradation douce. } \\
\text { - Assurance de perte d'exploitation. }\end{array}$ \\
\hline
\end{tabular}




\section{APPLICATION $N^{\circ} 20$ REGULATION DE LA DISTRIBUTION DES CARBURANTS}

Régulation en temps réel, et pour au moins $80 \%$ du réseau européen, de la distribution par pipelines des carburants (gaz, pétrole,...).

DATE DE REALISATION MOYENNE

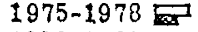

$1978-1983$

$1983-1988$

Après 1988

Jamais
Moyeme 1984

$1^{\circ}$ réponse

$2^{\circ}$ et $3^{\circ}$ réponses

\section{DATES AU PLUS TOT}

AU PLUS TARD

EN FONCTION DES EVENEMENTS SOCIO ECONOMQUES

Au plus tôt 1980

Au plus tard 1987

\section{COMMENTAII ES}

- Accélérations :

- Réglementation genérale ou hationale sur la distribution des carburants.

- Diminution par voie réglementalre, des marges de distribution, ce qui induira un effort d'optimisation des coats de transport des carburants.

- Augmentation sensible du cout des assurances sur le transport des carburants.

- Diminution du cout des équipements informatiques.

- Freins :

- Problènes de réglementations concernant l'environnement.

- Disponibilité de dispositifs d'analyse et de commandes fiables et robustes. 


\begin{tabular}{|c|c|c|}
\hline \multicolumn{3}{|c|}{ ANALYSE DFS PERTES POSSUBLIJ } \\
\hline $\begin{array}{l}\text { PERTES TCTALES ENVISAGLES } 63 . \\
\text { lion } \\
\text { Pertes attelgnant } 5 \times 10 \text { FS } .82,2 . \\
\text { Frequences et amplitude } \\
\text { Hautes frequence/Forte amplitude } \\
\text { Hautes frequence/Basse amplitude } \\
\text { Frequence fable/ Forte anplitude } \\
\text { Frequence falile/Basse amplitude } \\
\begin{aligned} \text { Dommages calises - hommes } \\
\text { - enireprise } \\
\text { - clionts } \\
\text { - autres }\end{aligned}\end{array}$ & $\begin{array}{l}\text { 25.mil- } \\
\text { S FS } \\
\ldots \ldots \% \\
\\
82,2 \\
17,8 \\
\\
33 \\
50,6 \\
10,1 \\
6,3\end{array}$ & $\begin{array}{l}\text { Sidurces d'incldents } \\
\text { - Equipements } \\
\text { - Logiciel } \\
\text { - Saisie } \\
\text { - Restitulion } \\
\text { - Erreurs utilisateurs } \\
\text { - Fraude - sabotage } \\
\text { - Divers }\end{array}$ \\
\hline Principales sources d'incidents & $\begin{array}{l}\text { Ir requence } \\
\text { et amplitu- } \\
\text { de moyonne } \\
\text { des incidents }\end{array}$ & $\begin{array}{l}\text { Proposition de methodes } \\
\text { de reduction de:s peries }\end{array}$ \\
\hline $\begin{array}{l}\text { Fonctionnement incorrect des van- } \\
\text { nes de régulation : } \\
\text { - pertes de produits par melan- } \\
\text { ge hors des normes } \\
\text { - coot de détection et de répara- } \\
\text { tion }\end{array}$ & $\begin{array}{l}1600 / \mathrm{an} \\
32.500 \mathrm{FS}\end{array}$ & $\begin{array}{l}\text { - Doublement des dispositifs de régu- } \\
\text { lation. } \\
\text { - Procédures de détection, par feed- } \\
\text { back, du non respect des ordres } \\
\text { envoyes. } \\
\text { - Systeme de commande manuel ou se- } \\
\text { mi automatique. }\end{array}$ \\
\hline $\begin{array}{l}\text { Le système tombe en panne au } \\
\text { moins une heure d'où : } \\
\text { - pertes de produits } \\
\text { - livraison de produits non con- } \\
\text { formes aux commandes. }\end{array}$ & $\begin{array}{c}15 / . \mathrm{an} \\
350.000 \mathrm{FS}\end{array}$ & $\begin{array}{l}\text { - Procédure de dégradation douce } \\
\text { a vec controle manuel. } \\
\text { - Doublement du processeur central } \\
\text { et/ou utilisation d'informatique } \\
\text { répartie. } \\
\text { - Maintenance préventive systématique } \\
\text { - Assurance perte d'exploitation. }\end{array}$ \\
\hline $\begin{array}{l}\text { Sabotage des equipements de contro- } \\
\text { le et de commande automatique des } \\
\text { vannes : } \\
\text { - perte de produits } \\
\text { - atteintes à la vie humaine par } \\
\text { conséquences indirectes. }\end{array}$ & $2 \underset{F S}{2 / a n}$ & $\begin{array}{l}\text { - Protection physique des } \\
\text { dispositifs critiques. } \\
\text { - Procédures de sauvegarde. }\end{array}$ \\
\hline & & \\
\hline
\end{tabular}




\section{APELICATION N' 24 CONTROLE DU TRAFIC URBAIN}

Dans au moins 15 grandes villes européennes, systèmes automatisés de régulation des transports de type privé par analyse systèmatique des différents flux de véhicules.

\section{ESTIMATIONS DU PANEL}

DATE DE REALISATION MOYENNE

1975-1978 口

$1978-1983$

$1983-1988$ ap

Après 1988 लूखि

Moyenne 1985

Jamais $\square$
DATES AU PLUS TOT

AU PLUS TARD

EN FONCTION DES EVENEMENTS

SOCIO ECONOMIQUES

Au plus tôt 1982

Au plus tard 1987

COMMENTAIRES

- Accélérations :

- Appui par les groupes de pression de l'industrie automoblle

- Investissements accordés par les pouvoirs publics.

- Détérioration du niveau de service offert par les transports publics.

- Freins :

- Trafic individuel de plus en plus difficile.

- Récession économique aggravée.

- Réglementation de l'utilisation de l'automobile pour diminuer les problèmes de pollution donc réduction des besoins en systèmes de régulation du trafic.

- Augmentation du niveau de service offert par les transports publics. 


\begin{tabular}{|c|c|c|}
\hline \multicolumn{3}{|c|}{ ANALYSE DES DERTES FOSSIBLES } \\
\hline 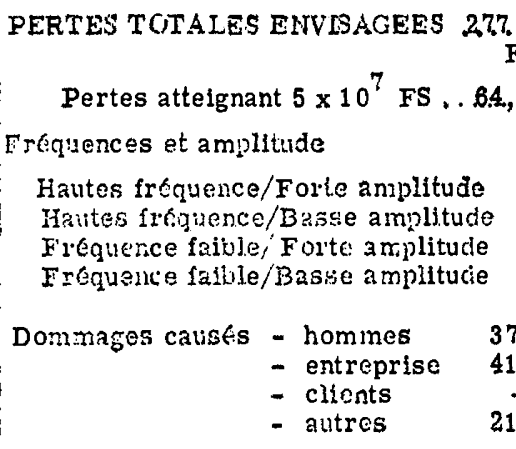 & $\begin{array}{l}\text { millions } \\
\text { FS } \% \\
95,2 \\
4,8 \\
7,3 \\
1,1 \\
1,6\end{array}$ & $\begin{array}{ll}\text { Sources d'inçidents } & \\
\text { - Equipements } & 4,8 \\
\text { - Logiciel } & \\
\text { - Saisio } & 19,6 \\
\text { - Restitution } & 28,8 \\
\text { - Erreurs utilisateurs } & 46,8 \\
\text { - Fraude - sabotage } & \\
\text { - Divers } & \end{array}$ \\
\hline Erincipiles sowices d'incidents & $\begin{array}{l}\text { Frequence } \\
\text { et. amplitid- } \\
\text { de movenue } \\
\text { des incidens }\end{array}$ & $\begin{array}{l}\text { Proposition de methodes } \\
\text { de réduction de's pertes }\end{array}$ \\
\hline $\begin{array}{l}\text { Le système tombe en panne au moins } \\
\text { une heure amenant : } \\
\text { - dommages matériels et corpo- } \\
\text { rels donc réparations financiè- } \\
\text { res. } \\
\text { - pertes de temps, retards pour } \\
\text { les usagers. }\end{array}$ & $87.500 \mathrm{FS}$ & $\begin{array}{l}\text { - Doublement des éléments critiques } \\
\text { - Procédures de dégradation douce } \\
\text { jusque et y compris système manuel } \\
\text { - Maintenance préventive. } \\
\text { - Assurance responsabilité civile. }\end{array}$ \\
\hline $\begin{array}{l}\text { La régulation est basée sur des in- } \\
\text { formations statistiques er ronées } \\
\text { d'oú : } \\
\text { - pertes de temps, retards pour } \\
\text { les usagers } \\
\text { - mauvaise image du servíce } \\
\text { public. }\end{array}$ & $\begin{array}{l}21.100 / \mathrm{an} \\
6.200 \mathrm{FS}\end{array}$ & $\begin{array}{l}\text { - Simulation du fonctionnement du sys- } \\
\text { téme. } \\
\text { - Essais en parallèle du nouveau sys- } \\
\text { tème pendant au moins } 3 \text { mois. }\end{array}$ \\
\hline $\begin{array}{l}\text { Fonctionnement dégradé des disposi- } \\
\text { tifs de saisle et des dispositifs de } \\
\text { cominandes : } \\
\text { - dommages matériels et corpo- } \\
\text { rels donc réparations financiè- } \\
\text { res. } \\
\text { pertes de temps. }\end{array}$ & $\begin{array}{l}1500 / \mathrm{an} \\
66.000 \mathrm{FS}\end{array}$ & $\begin{array}{l}\text { - Doublement des éléments critiques } \\
\text { - Tests permanents du fonctionnement } \\
\text { des dispositifs. } \\
\text { - Procédure de secours manuelles ou } \\
\text { semi automatique. } \\
\text { - Assurance responsabilité civile. }\end{array}$ \\
\hline $\begin{array}{l}\text { Panne des dispositifs de commande } \\
\text { de la circulation (feux...) } \\
\text { - pertes de temps } \\
\text { - mauvaise image du service } \\
\text { public } \\
\text { cout de réparation }\end{array}$ & $\begin{array}{l}4.600 / \mathrm{an} \\
7500 \mathrm{FS}\end{array}$ & $\begin{array}{l}\text { - Doublement des éléments critiques } \\
\text { - Maintenance préventive rigoureuse } \\
\text { - Procédures de secours manuelles } \\
\text { ou semi automatiques. }\end{array}$ \\
\hline
\end{tabular}


Pour les centres urbains européens de plus de 250.000 habitants, mesure des dif férents niveaux de pollution, prévision d'évolution à court et moyen terme (2 à 12 heu res) et déclenchement d'alerte s'il apparaft que les limites de sécurité vont être dépassées.

\section{ESTIMATIONS DU PANEI \\ DATE DE REALISATION MOYENNE}

$1975-1978$

1978-1983

$1983-1988$

Après 1988

Jamais
Moyenne 1982

$1^{\circ}$ réponse

$2^{\circ}$ et $3^{\circ}$ réponses
DATES AU PLUS TOT AU PLUS TARD

EN FONCTION DES EVENEMENTS SOCIO ECONOMIQUES

Au plus tốt 1980

Au plus tard 1985

\section{COMMENTAIRES}

- Accélérations :

- Senbilisation de l'opinion publique aux problèmes d'environnement et de pollution.

- Initiatives de type politique.

- Réglementations nationales ou internationales.

- Augmentation importante des crédits destinés à l'amélioration de l'environnement

- Freins :

- Coots de mise en oeuvre de ces systèmes.

- Difficultés de concevoir les logiciels nécessaires intégrant toutes les données.

- Disponibilité de capteurs peu coúteux et fiables. 


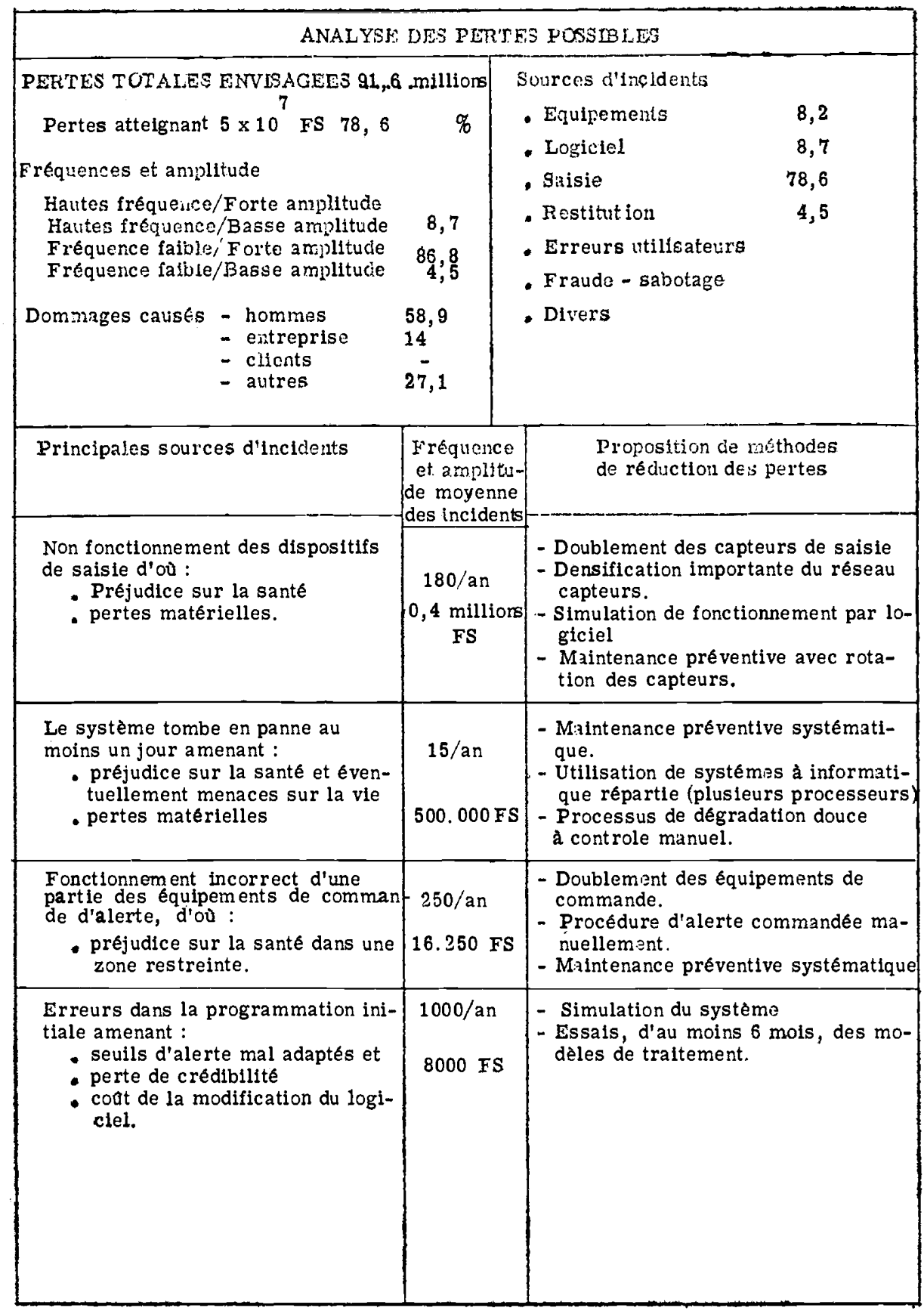




\section{APPLICATION N $N^{2} \quad 27$ BANQUE DE DONNEES INDIVIDUELLES}

Banque de données à l'échelon national concernant les individus et incluant tous les renseignements administratifs possibles à l'usage de l'administrationgouvernementale et locale.

ESTIMATIONS DU PANEI

DATE DE REALISATION MOYENNE

$1875-1978$

1978-1983

$1983-1988$

Après 1988

Jamais
Moyenne 1986

$1^{\circ}$ réponse

$2^{\circ}$ et $3^{\circ}$ réponses
DATES AU PLUS TOT AU PLUS TARD

EN FONCTION DES EVENEMENTS SOCIO ECONOMQUES

Au plus tôt

Au plus tard 1990

\section{COMMENTAIRES}

\section{- Accélération :}

- Réglementation concernant l'utilisation de l'informatique

- Deuntologie des informaticiens

- Développement de logiciel perməttant la détection de toute pénétration de la banque de données.

- Freins :

- Réglementation conceriant l'utilisation de l'informatique

- Deontologie des informaticiens

- Réglementation concernant les atteintes à la vie privée et le droit au secret pour chaque individu

- Rejet de l'informatisation, en particulier dans le domaine humain. 


\begin{tabular}{|c|c|c|}
\hline \multicolumn{3}{|c|}{ ANALYSI: DES PER'TES POSSIBLES } \\
\hline 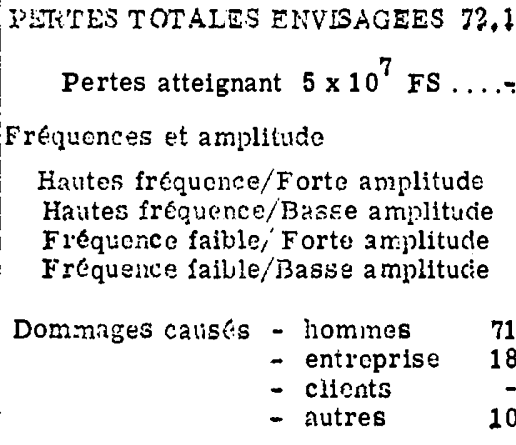 & 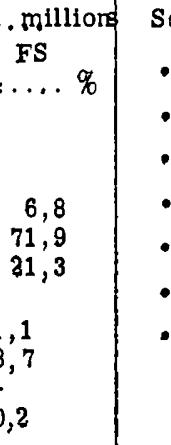 & $\begin{array}{l}\text { Sources d'inctdents } \\
\text { - Equipements } \\
\text { - Logieiel } \\
\text { - Saisie } \\
\text { - Restitut ion } \\
\text { - Erreurs utilisateurs } \\
\text { - Fraude - sabotage } \\
\text { - Divers }\end{array}$ \\
\hline Primcipales solices d'incidents & $\begin{array}{l}\text { Frequence } \\
\text { et. amplitis- } \\
\text { de mojenne } \\
\text { des incidents }\end{array}$ & $\begin{array}{l}\text { Proposition de methodes } \\
\text { de léduction des pertes }\end{array}$ \\
\hline $\begin{array}{l}\text { Sabotage ou fraude pendant la sortie } \\
\text { des informations résultantes d'ou : } \\
\text { - utilisation d'informations er- } \\
\text { ronnées } \\
\text { - dédommagements aux personnes } \\
\text { lésées. }\end{array}$ & $\begin{array}{l}20 \text { par an } \\
300.000 \mathrm{FS}\end{array}$ & $\begin{array}{l}\text { - Système de controle des informations } \\
\text { issues du systeme } \\
\text { - Définition de règles rigoureuses de } \\
\text { choix du personnel informatique } \\
\text { - Audit specifique tous les } 6 \text { mois. }\end{array}$ \\
\hline $\begin{array}{l}\text { Sabotage ou fraude pendant la saisie } \\
\text { des informations d'ou : } \\
\text { - cout de détection et de correc- } \\
\text { tion des erreurs. } \\
\text { - pertes d'informations. }\end{array}$ & $\begin{array}{c}175 / \mathrm{an} \\
40.000 \mathrm{FS}\end{array}$ & $\begin{array}{l}\text { - Definition de règles rigoureuses pour } \\
\text { le recrutement du persornel } \\
\text { - Controle systématique par sondages } \\
\text { - Audit specifique tous les } 6 \text { mois } \\
\text { - Controle sévère des informations } \\
\text { d'entrée. }\end{array}$ \\
\hline $\begin{array}{l}\text { Non fonctionnement des equipements } \\
\text { de saisie amenant : } \\
\text { - erreurs d'informations } \\
\text { - cout de détection et de correc- } \\
\text { tion des informations } \\
\text { - retards }\end{array}$ & $\begin{array}{l}? .700 / \mathrm{an} \\
1800 \mathrm{FS}\end{array}$ & $\begin{array}{l}\text { - Procédure de secours manuelles ou } \\
\text { semi manuelles } \\
\text {-Utilisation de terminaux et/ou de mo- } \\
\text { yens de saisie fiables. } \\
\text { - Doublement des dispositifs. }\end{array}$ \\
\hline $\begin{array}{l}\text { Erreurs dans la conception du logi- } \\
\text { ciel d'ou : } \\
\text { - altération des fichiers } \\
\text { - cout de détection et de réfection } \\
\text { du logiciel. } \\
\text { - dédommagements divers }\end{array}$ & $\begin{array}{l}5 \text { par an } \\
\text { FS } \\
\text { FS }\end{array}$ & $\begin{array}{l}\text { - Mise en route progressive des dif- } \\
\text { férentes fonctions du systeme } \\
\text { - Fonctionnement controle du nouveau } \\
\text { systeme pendant un an avant de déci- } \\
\text { der de son utilisation courante. } \\
\text { - Tests du systeme par simulation des } \\
\text { cas prévus. }\end{array}$ \\
\hline
\end{tabular}




\section{APPLICATION N $N^{\circ} 28$ ALERTE METEOROLOGIQUE}

Systeme général mondial d'informations météorologiques et de prévision (au moins cinq jours à l'avance) des évènements naturels graves (tremblements de terre, ouragans, raz de marée ...)

\begin{tabular}{|c|c|}
\hline 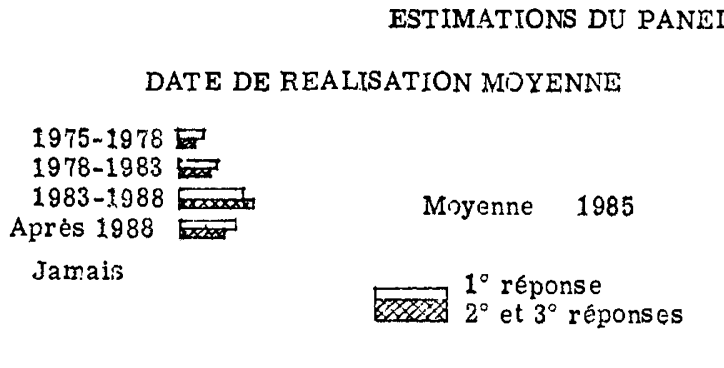 & $\begin{array}{l}\text { DATES AU PLUS TOT } \\
\text { AU PLUS TARD } \\
\text { EN FONCTION DES EVENEMENT: } \\
\text { SOCIO ECONOMQUUES } \\
\text { Au plus tot } \frac{1980}{1988} \\
\text { Au plus tard }\end{array}$ \\
\hline $\begin{array}{l}\text { COMMENTARRES } \\
\text { - Accélérations : } \\
\text { - Initiatives de type politique } \\
\text { - Entente internationale rapide sur les niveaux d } \\
\text { - Augmentation sensible des crédits. }\end{array}$ & tection et les seuils d'alerte. \\
\hline
\end{tabular}

- Freins :

- Entente internationale très lente sur les problèmes de détection et d'alerte.

- Coots de mise en oeuvre de ces systemes et leur mode de répartition.

- Mise au point de logiciel fiable. 


\begin{tabular}{|c|c|c|}
\hline \multicolumn{3}{|c|}{ ANALYSE DES PERTES IOSSTBLES } \\
\hline 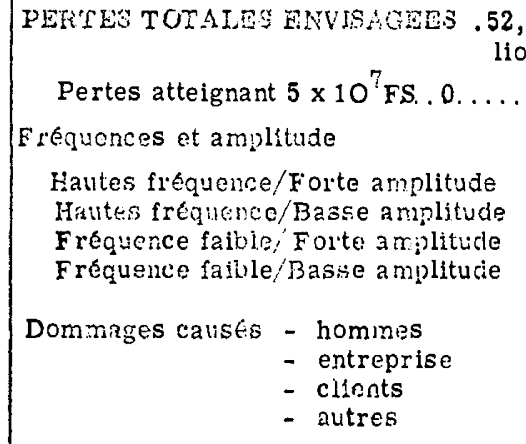 & $\begin{array}{c}6 . \mathrm{mil}- \\
\% \\
\\
\\
\\
92,4 \\
7,6 \\
29,1 \\
22 \\
24,6 \\
24,3\end{array}$ & 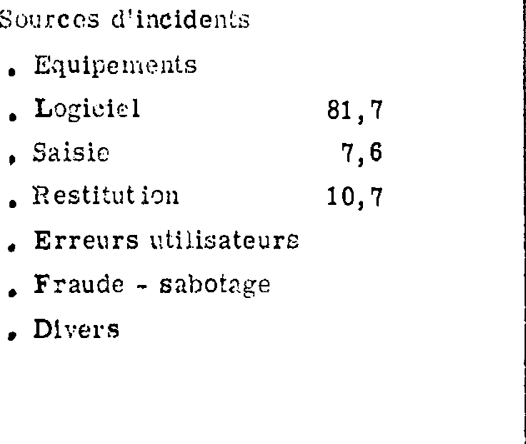 \\
\hline Principales sources d'incidents & $\begin{array}{l}\text { Ir requoncs } \\
\text { ef amplitu- } \\
\text { de moyenne } \\
\text { des incidents }\end{array}$ & $\begin{array}{l}\text { Proposition de rithodes } \\
\text { de réduction des pertes }\end{array}$ \\
\hline $\begin{array}{l}\text { Utilisation de logiciel périmé ame- } \\
\text { nant : } \\
\text { - alertes intempestives } \\
\text { - travaux à refaire }\end{array}$ & $\begin{array}{l}25 \text { par an } \\
1 \text { million } \\
\text { FS }\end{array}$ & $\begin{array}{l}\text { - Organisation rigoureuse de l'exploi- } \\
\text { tation du système. } \\
\text { - Formation poussée des operateurs } \\
\text { - Développement par le constructeur, } \\
\text { de systèmes opératoires totalement } \\
\text { fiables. }\end{array}$ \\
\hline $\begin{array}{l}\text { Non fonctionnement ou fonctionne- } \\
\text { ment incorrect des dispositifs de } \\
\text { saisie : } \\
\text { - retards dans l'alerte } \\
\text { - cout de remise en oeuvre }\end{array}$ & $\begin{array}{r}40 \text { par an } \\
100.000 \mathrm{FS}\end{array}$ & $\begin{array}{l}\text { - Doublement des dispositifs de saisie } \\
\text { - Maintenance préventive rigoureuse } \\
\text { - Tests permanents de vérification du } \\
\text { fonctionnement. }\end{array}$ \\
\hline $\begin{array}{l}\text { Non fonctionnement des équipements } \\
\text { d'alerte : } \\
\quad \text { - pertes matérielles et humaines }\end{array}$ & $\begin{array}{l}2 \text { par an } \\
2,8 \text { milliors } \\
\text { FS }\end{array}$ & $\begin{array}{l}\text { - Doublement des équipements d'alerte } \\
\text { - Procédures impérative de } \\
\text { secours permettant l'alerte manuelle } \\
\text { - Maintenance préventive. }\end{array}$ \\
\hline $\begin{array}{l}\text { Algorithmes de traitements pas } \\
\text { assez précis: } \\
\text { - seuils d'alerte mal adaptés } \\
\text { (soit alerte trop fréquente soit } \\
\text { trop tardive) } \\
\text { - cout de modification du logiciel }\end{array}$ & $\begin{array}{l}10 \text { par an } \\
1,8 \text { millions } \\
\text { FS }\end{array}$ & $\begin{array}{l}\text { - Longue simulation du système } \\
\text { - Mise en route progressive du systè- } \\
\text { me avec contrôle pas à pas. }\end{array}$ \\
\hline
\end{tabular}


Au moins $75 \%$ des villes européennes de plus de 10.000 habitants utiliseront un système de gestion (comptabilité, impots, recensement, problèmes fonciers) basé sur ordinateur.

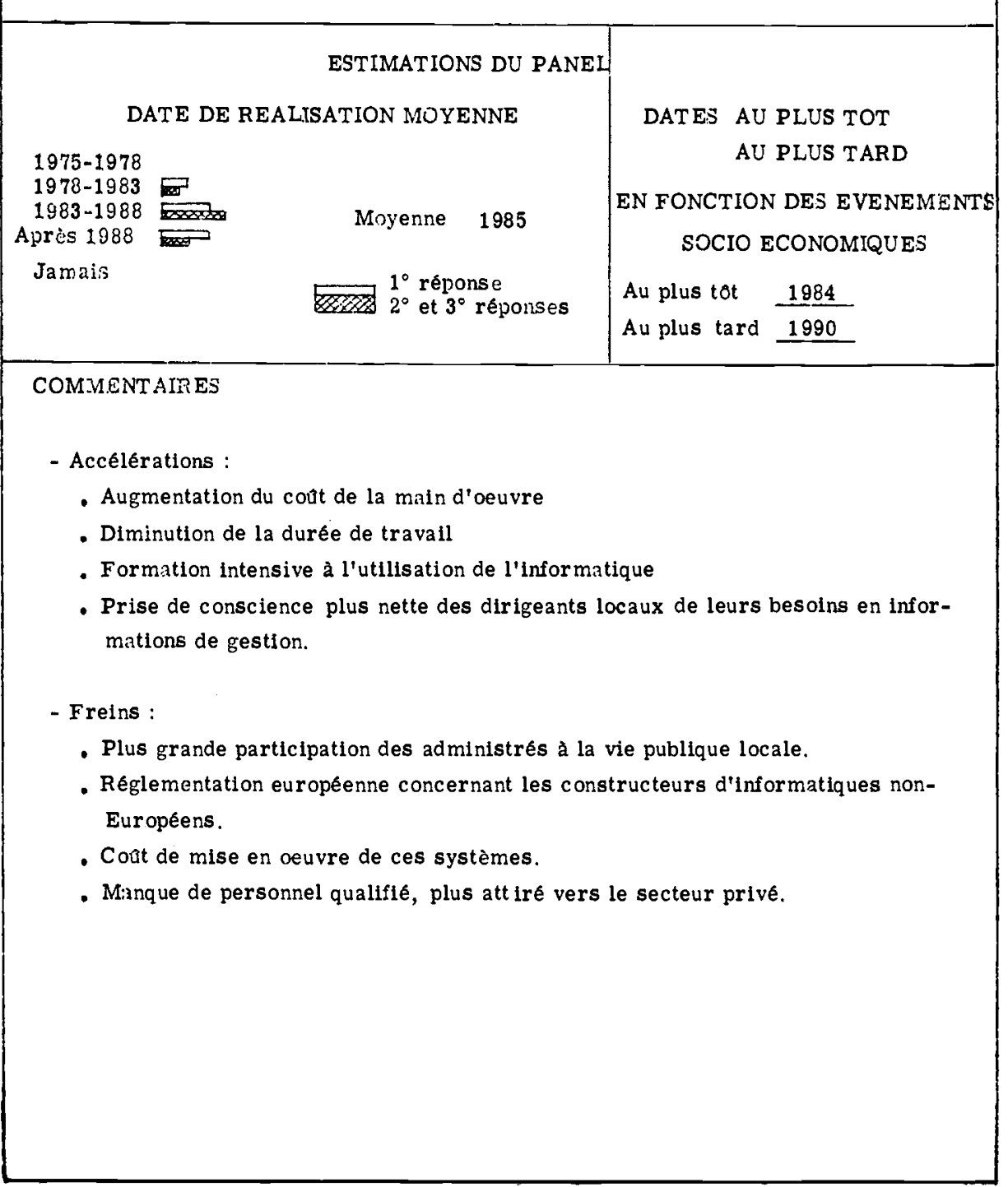




\begin{tabular}{|c|c|c|}
\hline \multicolumn{3}{|c|}{ ANALYSY DOS YURSW POSSUBLES } \\
\hline 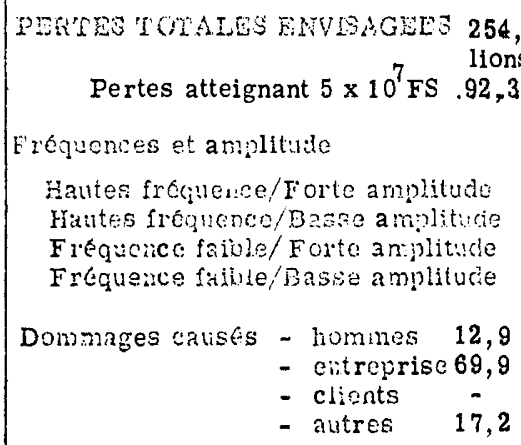 & $\begin{array}{l}\text { 6.mil- } \\
\text { is FS } \\
3 \% \% \\
\\
27,5 \\
71,8 \\
0,7\end{array}$ & $\begin{array}{l}\text { Sources d'incidents } \\
\text { - Equipenents } \\
\text { - Loriciel } \\
\text { - Saisie } \\
\text { - Restitution } \\
\text { - Erreurs utilisiateurs } \\
\text { - Fraude - sabotage } \\
\text { - Divers (exploitation) }\end{array}$ \\
\hline Trincipules suirces cincicients & $\begin{array}{l}\text { Frequonce } \\
\text { et amplitu- } \\
\text { de moycnne } \\
\text { des incidents }\end{array}$ & $\begin{array}{l}\text { Proposition die mithodes } \\
\text { de reduction des pertes }\end{array}$ \\
\hline $\begin{array}{l}\text { Non fonctionnement des equipements } \\
\text { de saisie : } \\
\text { - retards } \\
\text { - cout de correction }\end{array}$ & $\begin{array}{c}100.000 / \mathrm{an} \\
700 \mathrm{FS}\end{array}$ & $\begin{array}{l}\text { - Procèdures de secours manuelles ou } \\
\text { semi manuelles. } \\
\text { - Doublement des dispositifs de saisie } \\
\text { et maintenance preventive. } \\
\text { - Formalisation des procèdures de tra } \\
\text { vail et formation poussée des utilisa- } \\
\text { teurs. }\end{array}$ \\
\hline $\begin{array}{l}\text { Erreurs dans la manipulation des } \\
\text { divers fichiers et programmes } \\
\text { amenant: } \\
\text { - retards et pertes de temps. } \\
\text { - cout de correction des erreurs } \\
\text { - dédommagement des tiers. }\end{array}$ & $\begin{array}{c}600 / \mathrm{an} \\
7.75 .000 \mathrm{FS}\end{array}$ & $\begin{array}{l}\text { - Controle strict des règles de produc } \\
\text { tion ordinateur. } \\
\text { - Formation poussé du personnel } \\
\text { d'exploitation. } \\
\text { - Assurance perte d'exploitation. }\end{array}$ \\
\hline $\begin{array}{l}\text { Le systeme tombe en panne au } \\
\text { moins une heure d'où : } \\
\text { - arrets et retards dans les } \\
\text { travaux. } \\
\text { - cout de remise en route. }\end{array}$ & $\begin{array}{l}30 / \mathrm{an} \\
52.000 \mathrm{FS}\end{array}$ & $\begin{array}{l}\text { - Procèdures de dépannage et de se- } \\
\text { cours. } \\
\text { - Assurance perte d'exploitation. } \\
\text { - Doublement des équipements essen- } \\
\text { tiels. }\end{array}$ \\
\hline $\begin{array}{l}\text { Sabotage ou fraude amenant : } \\
\text { - pertes financières directes } \\
\text { - couts de remise en oeuvre du } \\
\text { systeme. }\end{array}$ & $\begin{array}{c}30 / \mathrm{an} \\
600.000 \mathrm{FS}\end{array}$ & $\begin{array}{l}\text { - Audit des systèmes informatiques } \\
\text { - Rotation du personnel. } \\
\text { - Système de controle croisé par type } \\
\text { d'application. }\end{array}$ \\
\hline
\end{tabular}




\section{APPLICATION N $N^{\circ} 34$ LABORATOIRE D'ANALYSES MEDICALES AUTOMATIQUES}

Systèmes utilisés dans au moins $50 \%$ des laboratoires médicaux pour effectuer automatiquement des anakyses chimiques (dosage 'd'urée sanguine) et des analyses physiques (EEC et EEG).

ESTIMATIONS DU PANEL

DATE DE REALISATION MOYENNE

$1975-19780$

1978-1983 चax

1983-1988

Après 1988

Jamais
Moyenne 1984

$1^{\circ}$ réponse

$2^{\circ}$ et $3^{\circ}$ réponses
DATES AU PLUS TOT

AU PLUS TARD

EN FONCTION DES EVENEMENTS SOCIO ECONOMLUES

Au plus tot 1981

Au plus tard 1989

\section{COMMENTAIRES}

- Accélérations :

- Mise à disposition d'équipements fiables et moins coateux

- Développement du logiciel nécessaire.

- Disponibilité de bases de données fiables et standards.

- Concentration des laboratoires d'analyses médicales.

- Freins :

- Coút de développement de ces systèmies

- Acceptation par la profession

- Disponibilité d'équipements fiables et peu couteux.

- Mise au point d'analyseurs très sensibles. 


\begin{tabular}{|c|c|c|}
\hline \multicolumn{3}{|c|}{ ANALYSE DES PERTES POSSIBLES } \\
\hline 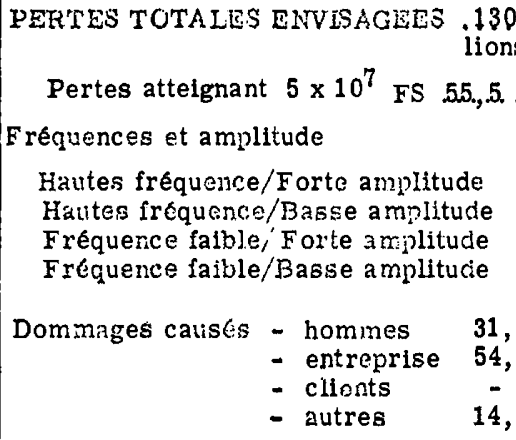 & $\begin{array}{l}\text { is mil- } \\
\text { is } \\
8 \\
84,6 \\
4,7 \\
10,7 \\
2 \\
7 \\
1\end{array}$ & $\begin{array}{l}\text { Sources d'incidents } \\
\text { - Equipenients } \\
\text { - Logiciel } \\
\text { - Saisie } \\
\text { - Restitution } \\
\text { - Erreurs utilisateurs } \\
\text { - Fraude - sabotage } \\
\text { - Divers }\end{array}$ \\
\hline Principales sources d'incidents & $\begin{array}{l}\text { Frequence } \\
\text { et amplitu- } \\
\text { de moyenne } \\
\text { des incidents }\end{array}$ & $\begin{array}{l}\text { Proposition de methodes } \\
\text { de reduction des pertes }\end{array}$ \\
\hline $\begin{array}{l}\text { Le système tombe en panne au } \\
\text { moins une heure d'où } \\
\text { - perte de production } \\
\text { - retards et dédommagements } \\
\text { eventuels aux clients. }\end{array}$ & $\begin{array}{c}170 / \text { an } \\
82.500 \mathrm{FS}\end{array}$ & $\begin{array}{l}\text { - Procedures de dégradation douce et/ } \\
\text { ou utilisation d'informatique répartie } \\
\text { - Maintenance préventive. } \\
\text { - Doublement des processeurs centrau } \\
\text { - Assurance perte d'exploitation. }\end{array}$ \\
\hline $\begin{array}{l}\text { Non fonctionnement des équipements } \\
\text { de saisie: } \\
\text { - retards en production } \\
\text { - pertes d'informations } \\
\text { - coot de correction. }\end{array}$ & $\begin{array}{c}950 / \mathrm{an} \\
40.000 \mathrm{FS}\end{array}$ & $\begin{array}{l}\text { - Doublement des unités de saisie. } \\
\text { - Procédures manuelles d'entrée dans } \\
\text { le système. } \\
\text { - Formalisation des procèdures de tra- } \\
\text { vail et formation des utilisateurs. }\end{array}$ \\
\hline $\begin{array}{l}\text { Non fonctionnement des dispositifs } \\
\text { de sortie: } \\
\text { - pertes d'exploitation } \\
\text { - coat de remise en oeuvre. }\end{array}$ & $\begin{array}{l}2000 / \mathrm{an} \\
36.250 \mathrm{FS}\end{array}$ & $\begin{array}{l}\text { - Doublement des dispositifs de sortie } \\
\text { - Procedures de secours manuelles ou } \\
\text { semi automatiques. } \\
\text { - Maintenance préventive } \\
\text { - Assurance Responsabilité civile et } \\
\text { Perte d'exploitation. }\end{array}$ \\
\hline $\begin{array}{l}\text { Erreurs dans la conception du lo- } \\
\text { giciel : } \\
\text { - pertes d'exploitation } \\
\text { - perte de clientele } \\
\text { - dédommagements divers } \\
\text { - coat de correction. }\end{array}$ & $\begin{array}{c}16 / \mathrm{an} \\
380.000 \mathrm{FS}\end{array}$ & $\begin{array}{l}\text { - Longue période (au moins } 6 \text { mois) } \\
\text { d'essais du système. } \\
\text { - Fonctionnement en parallèle du nou- } \\
\text { veau et de l'ancien systeme pendant } \\
3 \text { mois. } \\
\text { - Mise en route progressive des diffé- } \\
\text { rentes fonctions. }\end{array}$ \\
\hline
\end{tabular}




\section{APPLICATION No 38 SYSTEMES D'ENSEIGNEMENT INDIVIDUALISE}

Plus de 25 écoles européennes de niveau supérieur auront un système d'enseignement individuel basé sur la méthode d'enseignement programmé avec controle individuel de la progression des connaissances.

ESTIMATIONS DU PANEL

DATE DE REALISATION MOYENNE

1975-1978ם

$1978-1983$ 国

$1983-1988$ Apras

Après 1988

Jamais
口
Moyenne 1986

$1^{\circ}$ réponse

\section{DATES AU PLUS TOT}

AU PLUS TARD

EN FONCTION DES EVENEMENTS SOCIO ECONOMIQUES

Au plus tot 1983

Au plus tard 1990

COMMENT AIRES

- Accélérations :

- Faillite du système d'éducation actuelle.

- Mise au point et développement extensif des procédures d'enseignement programmé adaptées à l'enseignement supérieur.

- Diminution du coot des équipements informatiques.

- Freins :

- Réticences, voires répugnances, des étudiants à utiliser un système informatique.

- Coot de mise en oeuvre.

- Grande vulnérabilité de systemes de ce type. 


\begin{tabular}{|c|c|c|c|}
\hline \multicolumn{4}{|c|}{ ANALYSE DES PERTES FUSSD 3 LES } \\
\hline 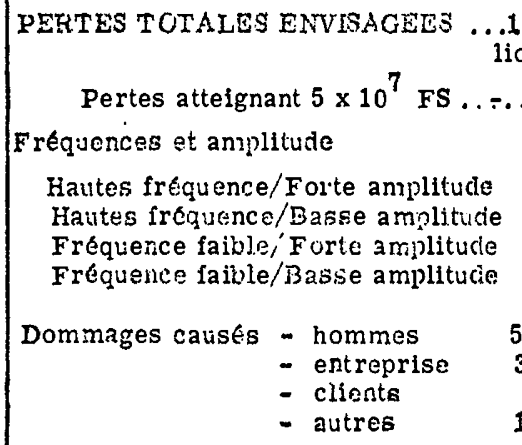 & 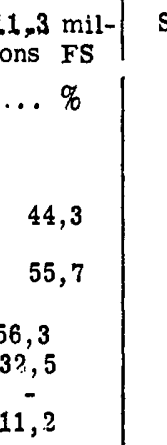 & $\begin{array}{l}\text { Sources d'incidents } \\
\text { - Eruipemonts } \\
\text { - Logiciel } \\
\text { - Saisie } \\
\text { - Restitution } \\
\text { - Errours utilisateurs } \\
\text { - Fraude - sabotage } \\
\text { - Divers }\end{array}$ & $\begin{array}{l}19,5 \\
12,4 \\
44,3 \\
23,8\end{array}$ \\
\hline Principales scrirces d'inciclents & $\begin{array}{l}\text { Fréquence } \\
\text { et amplitu- } \\
\text { de moycune } \\
\text { des incidents }\end{array}$ & $\begin{array}{l}\text { Proposition de } \\
\text { de réduction de: }\end{array}$ & $\begin{array}{l}\text { hodes } \\
\text { irtes }\end{array}$ \\
\hline $\begin{array}{l}\text { Le système tombe en panne au } \\
\text { moins une heure d'où : } \\
\text { - pertes de temps } \\
\text { - coot de réparation } \\
\text { - perte de confiance dans ce sys- } \\
\text { tème }\end{array}$ & $\begin{array}{c}35 / \mathrm{an} \\
6 ? 500 \mathrm{FS}\end{array}$ & $\begin{array}{l}\text { - Prévoir procédures } \\
\text { douce. } \\
\text { - Utiliser l'informatic } \\
\text { le chamage en rése } \\
\text { - Maintenance préver }\end{array}$ & $\begin{array}{l}\text { dégradation } \\
\text { répartie ou } \\
\text { rigoureuse }\end{array}$ \\
\hline $\begin{array}{l}\text { Non fonctionnemsnt des équipe - } \\
\text { ments de sortie : } \\
\text { - pertes de temps } \\
\text { - mauvaise image du système } \\
\text { - coot de réparation }\end{array}$ & $\begin{array}{l}150 / \mathrm{an} \\
8.750 \mathrm{FS}\end{array}$ & $\begin{array}{l}\text { - Procédures de seco } \\
\text { - Doublement des dis } \\
\text { - Maintenance préven }\end{array}$ & $\begin{array}{l}\text { itifs de sortie } \\
\text { e rigoureuse. }\end{array}$ \\
\hline $\begin{array}{l}\text { Erreur dans l'utilisation du systè- } \\
\text { me d'où: } \\
\text { - pertes de temps } \\
\text { coot de remise en oeuvre. }\end{array}$ & $\begin{array}{l}5000 / \mathrm{an} \\
1000 \mathrm{FS}\end{array}$ & $\begin{array}{l}\text { - Formation poussée } \\
\text { - Utilisation de proce } \\
\text { de correction des }\end{array}$ & $\begin{array}{l}\text { utilisateurs } \\
\text { es simples } \\
\text { urs. }\end{array}$ \\
\hline $\begin{array}{l}\text { Sabotage du système amenant: } \\
\text { - pertes financières } \\
\text { coot de remise en oeuvre du } \\
\text { système. }\end{array}$ & $\begin{array}{c}100 / \mathrm{an} \\
\text { ?7.500 FS }\end{array}$ & $\begin{array}{l}\text { - Protection physique } \\
\text { critiques. } \\
\text { - Procédures de sauv } \\
\text { Démystification des } \\
\text { l'informatique chez } \\
\text { de système. }\end{array}$ & $\begin{array}{l}\text { s dispositifs } \\
\text { rde } \\
\text { ntraintes de } \\
\text { s utilisateurs }\end{array}$ \\
\hline
\end{tabular}




\section{Annexe B : Evolution technique et technologique}

\section{Considérations générales sur les systèmes informatiques}

\begin{tabular}{|c|c|c|c|}
\hline \multirow{2}{*}{ Désignation de l'événement } & \multicolumn{3}{|c|}{$\begin{array}{l}\text { Probabilité d'aboutissement } \\
\text { de l'événement }\end{array}$} \\
\hline & Av. 1980 & $1980-1988$ & Après \\
\hline $\begin{array}{l}\text { 1. Disponibilité en Europe } \\
\text { de Réseaux Polyprocesseurs: }\end{array}$ & & \multirow[t]{2}{*}{0,4} & \multirow{2}{*}{0,6} \\
\hline $\begin{array}{l}\text { Ensemble de ressources variées (de saisie, traite- } \\
\text { ment, stockage et restitution de l'information) } \\
\text { entrelacées de telle sorte qu'on optimise l'utili- } \\
\text { sation de ces ressources à tout moment sans aucun } \\
\text { arrêt de service. }\end{array}$ & & & \\
\hline $\begin{array}{l}\text { 2. Disponibilité d'un ensemble de moyens hardware } \\
\text { et software pour assurer la sécurité totale d'une } \\
\text { banque de données. }\end{array}$ & 0,5 & 0,4 & 0,1 \\
\hline $\begin{array}{l}\text { 3. Systèmes complexes dont les terminaux éloignés } \\
\text { pourront être des robots-manipulateurs physiques } \\
\text { pouvant accomplir sur commande programmée } \\
\text { des tâches variées et précises. }\end{array}$ & 0,4 & 0,5 & 0,1 \\
\hline $\begin{array}{l}\text { 4. Fonctionnement normal de systèmes d'informa- } \\
\text { tique répartis dans les entreprises ou organismes } \\
\text { de grande taille. }\end{array}$ & 0,4 & 0,4 & 0,2 \\
\hline
\end{tabular}

2. Technologie de base et unités centrales

\begin{tabular}{|c|c|c|c|}
\hline \multirow[t]{2}{*}{ Désignation de l'événement } & \multicolumn{3}{|c|}{$\begin{array}{l}\text { Probabilité d'aboutissement } \\
\text { de l'événement }\end{array}$} \\
\hline & Av. 1980 & $1980-1988$ & Après \\
\hline $\begin{array}{l}\text { 5. Utilisation intensive du firmware (au moins la } \\
\text { moitié des fonctions microprogrammées). }\end{array}$ & 0,5 & 0,5 & \\
\hline $\begin{array}{l}\text { 6. Décroissance du ratio cô̂t/performance des } \\
\text { unités centrales d'un à deux ordres de grandeur. }\end{array}$ & 0,3 & 0,7 & \\
\hline $\begin{array}{l}\text { 7. Unités centrales autocorrectives dans trois quarts } \\
\text { des cas de panne par détection des éléments } \\
\text { défaillants et commutation automatique sur des } \\
\text { éléments sains. }\end{array}$ & 0,7 & 0,3 & \\
\hline $\begin{array}{l}\text { 8. Elimination totale des pannes et incidents par } \\
\text { extension des possibilités décrites à la rubrique } \\
\text { précédente. }\end{array}$ & 0,4 & 0,5 & 0,1 \\
\hline $\begin{array}{l}\text { 9. Rupture du «mur de la vitesse » créée par les } \\
\text { contraintes technologiques actuelles dues à la } \\
\text { longueur d'onde et au niveau d'énergie des } \\
\text { électrons }\end{array}$ & 0,1 & 0,3 & 0,6 \\
\hline
\end{tabular}




\begin{tabular}{|c|c|c|c|}
\hline \multirow[t]{2}{*}{ Désignation de l'événement } & \multicolumn{3}{|c|}{$\begin{array}{l}\text { Probabilité d'aboutissement } \\
\text { de l'événement }\end{array}$} \\
\hline & Av. 1980 & $1980-1988$ & Après \\
\hline $\begin{array}{l}\text { 10. Dispositifs de stockage aléatoire de l'information } \\
\text { d'au moins } 10^{11} \text { caractères accessibles en environ } \\
1 \text { seconde. }\end{array}$ & 0,6 & 0,4 & \\
\hline $\begin{array}{l}\text { 11. Disponibilité commerciale de mémoires de type } \\
\text { holographique. }\end{array}$ & 0,3 & 0,6 & 0,1 \\
\hline $\begin{array}{l}\text { 12. Systèmes de stockage basés sur la technique } \\
\text { microfilm (COM) de } 30000 \text { caractères au } \mathrm{cm}^{2} \\
\text { avec détection par faisceau laser. }\end{array}$ & 0,2 & 0,5 & 0,3 \\
\hline $\begin{array}{l}\text { 13. Commercialisation de mémoires associatives d'au } \\
\text { moins } 108 \text { caractères avec un temps de réponse } \\
\text { de l'ordre de la microseconde. }\end{array}$ & 0,3 & 0,7 & \\
\hline
\end{tabular}

\section{Input - output devices - Dispositifs entrée - sortie}

\begin{tabular}{|c|c|c|c|}
\hline \multirow{2}{*}{ Désignation de l'événement } & \multicolumn{3}{|c|}{$\begin{array}{l}\text { Probabilité d'aboutissement } \\
\text { de l'événement }\end{array}$} \\
\hline & Av. 1980 & $1980-1988$ & Après \\
\hline $\begin{array}{l}\text { 14. Ecrans à affichage alphanumérique à un coût } \\
\text { voisin du coût actuel des télétypes. }\end{array}$ & 0,8 & 0,2 & \\
\hline $\begin{array}{l}\text { 15. Ecrans à dispositif d'affichage graphique à un } \\
\text { coât voisin du coût actuel des écrans à affichage } \\
\text { alphanumérique. }\end{array}$ & 0,7 & 0,3 & \\
\hline $\begin{array}{l}\text { 16. Dispositifs de présentation des données de sortie } \\
\text { des systèmes informatiques en trois dimensions. }\end{array}$ & 0,6 & 0,4 & \\
\hline $\begin{array}{l}\text { 17. Dispositifs de lecture optique manuscrite (tels } \\
\text { qu'utilisables dans le tri postal) avec un taux de } \\
\text { rejet inférieur à } 5 \% \text {. }\end{array}$ & 0,5 & 0,5 & \\
\hline $\begin{array}{l}\text { 18. Dispositifs hardware de reconnaissance des for- } \\
\text { mes, capables de reconnaître les formes et objets } \\
\text { en trois dimensions. }\end{array}$ & 0,1 & 0,8 & 0,1 \\
\hline $\begin{array}{l}\text { 19. Disponibilité commerciale de dispositifs hardware } \\
\text { capables d'identifier l'utilisateur (par la voix) } \\
\text { sans risque d'erreur. }\end{array}$ & 0,3 & 0,7 & \\
\hline $\begin{array}{l}\text { 20. Développement de systèmes d'entrée vocale capa- } \\
\text { bles de produire une série d'informations digitales } \\
\text { à partir d'un texte alphanumérique avec un taux } \\
\text { d'erreur acceptable. }\end{array}$ & 0,1 & 0,7 & 0,2 \\
\hline
\end{tabular}


4. Input - output devices - Dispositifs entrée - sortie (suite)

\begin{tabular}{|c|c|c|c|c|}
\hline \multirow{2}{*}{\multicolumn{2}{|c|}{ Désignation de l'événement }} & \multicolumn{3}{|c|}{$\begin{array}{l}\text { Probabilité d'aboutissement } \\
\text { de l'événement }\end{array}$} \\
\hline & & Av. 1980 & $1980-1988$ & Après \\
\hline 21. & $\begin{array}{l}\text { Disponibilité commerciale d'un système de sortie } \\
\text { vocale capable de fournir un texte alphanu- } \\
\text { mérique. }\end{array}$ & 0,1 & 0,5 & 0,4 \\
\hline 22. & $\begin{array}{l}\text { Imprimantes sans impact à haute vitesse (10000 } \\
\text { lignes/minute). }\end{array}$ & 0,9 & 0,1 & \\
\hline 23. & $\begin{array}{l}\text { Dispositifs de sortie en fac-similé au prix actuel } \\
\text { d'un télétype et capables d'imprimer une page } \\
\text { en moins d'une minute. }\end{array}$ & 0,6 & 0,4 & \\
\hline 24. & $\begin{array}{l}\text { Disponibilité commerciale de terminaux «d'équi- } \\
\text { pement de foyer» pour environ } 500 \$ \text { de prix de } \\
\text { vente avec combiné téléphonique, clavier, petit } \\
\text { écran video et lecteur de badges. }\end{array}$ & 0,4 & 0,6 & \\
\hline & $\begin{array}{l}\text { Dispositifs de lecture optique capables de lire la } \\
\text { majorité des types de caractères existants à un } \\
\text { coût inférieur de } 50 \% \text { au coût actuel. }\end{array}$ & 0,6 & 0,4 & \\
\hline & $\begin{array}{l}\text { Réduction de coût de la saisie «clavier-bandes } \\
\text { ou disques magnétiques 》 d'environ } 30 \% \text {. }\end{array}$ & 0,4 & 0,6 & \\
\hline
\end{tabular}

\section{Communications}

\begin{tabular}{c|c|c|c}
\hline \multicolumn{1}{c|}{ Désignation de l'événement } & \multicolumn{2}{|c}{$\begin{array}{c}\text { Probabilité d'aboutissement } \\
\text { de l'événement }\end{array}$} \\
& Av. 1980 & $1980-1988$ & Après \\
\hline \begin{tabular}{l|l|c} 
27. Réseaux de commutation par paquets accessibles \\
en Europe par n'importe quel utilisateur.
\end{tabular} & 0,2 & 0,6 & 0,2 \\
$\begin{array}{l}\text { 28. Abaissement des coûts de communications digi- } \\
\text { tales par un facteur 5 en Europe. }\end{array}$ & 0,3 & 0,5 & 0,2 \\
$\begin{array}{l}\text { 29. En Europe, réseau téléphonique commuté à larges } \\
\text { bandes (2,5 mégahertz/seconde) permettant simul- } \\
\text { tanément l'utilisation au domicile d'un video et } \\
\text { de transmissions digitales. }\end{array}$ & 0,5 & 0,5 \\
\end{tabular}




\begin{tabular}{|c|c|c|c|c|}
\hline \multirow{2}{*}{\multicolumn{2}{|c|}{ Désignation de l'événement }} & \multicolumn{3}{|c|}{$\begin{array}{l}\text { Probabilité d'aboutissement } \\
\text { de l'événement }\end{array}$} \\
\hline & & Av. 1980 & $1980-1988$ & Après \\
\hline 30. & $\begin{array}{l}\text { Simplification dans la définition et la mise en } \\
\text { œuvre des systèmes d'exploitation pour les grands } \\
\text { systèmes. }\end{array}$ & 0,4 & 0,5 & 0,1 \\
\hline 31. & $\begin{array}{l}\text { Remplacement des compilateurs (type COBOL, } \\
\text { FORTRAN) par des modules hardware capa- } \\
\text { bles d'effectuer la traduction des programmes } \\
\text { utilisateurs. }\end{array}$ & 0,6 & 0,4 & \\
\hline 32. & $\begin{array}{l}\text { Langages de programmation plus simplifiés des- } \\
\text { tinés aux utilisateurs non informaticiens. }\end{array}$ & 0,6 & 0,3 & 0,1 \\
\hline 33. & $\begin{array}{l}\text { Langages de programmation utilisant la structure } \\
\text { et la syntaxe du langage courant anglais. }\end{array}$ & 0,2 & 0,6 & 0,2 \\
\hline 34. & $\begin{array}{l}\text { Programmation automatique à partir d'organi- } \\
\text { grammes standards. }\end{array}$ & 0,2 & 0,7 & 0,1 \\
\hline 35. & $\begin{array}{l}\text { Software résidant dans les systèmes informatiques } \\
\text { permettant l'adaptation des traitements aux } \\
\text { variations de conditions. }\end{array}$ & 0,4 & 0,6 & \\
\hline
\end{tabular}

Evolution du mode d'acquisition pour le parc installé (pourcentage basé sur la valeur du parc)

\section{Location}

Leasing

Achat

\begin{tabular}{|c|c|r|r|r|c}
\hline \multicolumn{3}{|c|}{ U.S.A. } & \multicolumn{3}{|c}{ EUROPE } \\
\hline 1970 & 1978 & 1988 & 1970 & 1978 & 1988 \\
\hline 50 & 48 & 46 & 61 & 62 & 55 \\
19 & 22 & 26 & 4 & 9 & 18 \\
31 & 30 & 28 & 35 & 29 & 27
\end{tabular}

Evolution P.N.B. (1970-1983)

en milliards $\$$ constants

\begin{tabular}{l|r|r|r|r|l}
\cline { 2 - 6 } & 1970 & 1975 & 1980 & 1983 & 1988 \\
\hline Europe & 846 & 1045 & 1225 & 1335 & 1420 \\
U.S.A. & 713 & 884 & 1040 & 1165 & 1230
\end{tabular}


Ventes annuelles en Europe (billions francs suisses)

\begin{tabular}{l|c|c|c|c|c}
\cline { 2 - 5 } & 1970 & 1973 & 1978 & 1983 & 1988 \\
\hline Software - Services & 2,9 & 4,2 & 9,5 & 15,9 & 26 \\
$\begin{array}{l}\text { Transmissions périphé- } \\
\text { riques offline }\end{array}$ & 1,8 & 3,1 & 7,9 & 16 & 29 \\
Ordinateurs & 6,9 & 9,2 & 15,9 & 26,5 & 32
\end{tabular}

Valeur des systèmes informatiques installés

(en billions francs suisses)

\begin{tabular}{l|r|r|r|r|r|r}
\cline { 2 - 7 } & 1960 & 1970 & 1973 & 1978 & 1983 & 1988 \\
\hline U.S.A. & 5,5 & 57,9 & 77,6 & 121 & 189 & 252 \\
Europe Ouest & 1,6 & 25,3 & 40,8 & 78 & 140 & 200 \\
Japon & 0,3 & 4,7 & 10,5 & 21 & 31 & 48 \\
Autres pays & 0,5 & 6 & 18,4 & 45 & 80 & 122 \\
\hline Total & 7,9 & 93,9 & 147,3 & 265 & 440 & 622 \\
\hline
\end{tabular}

Pour le monde, par type de produit en \%

\begin{tabular}{l|c|c|c|c|c|c} 
Ordinateurs & 75 & 63 & 58 & 43 & 35 & 31 \\
Périphériques associés & 22 & 26 & 30 & 40 & 40 & 38 \\
Transmission & 0,6 & 7,5 & 9 & 15 & 24 & 30 \\
\hline
\end{tabular}

\section{Annexe C : Fraudes et sabotages dans les systèmes informatiques}

Depuis les premières livraisons d'ordinateurs, la sécurité des systèmes d'informations a attiré l'attention des responsables, d'abord aux Etats-Unis, puis ensuite en Europe.

La pénétration rapide de l'informatique, dans le secteur privé aussi bien que public ces quinze dernières années, et l'importance croissante du nombre de travailleurs en relation directe ou indirecte avec les ordinateurs $(8-10 \%$ de la population active aux Etats-Unis, 5-6\% en Europe occidentale), joint aux malaises de la société actuelle, ont amené un développement rapide des «crimes informatiques». 


\section{Considérations générales}

Par "crimes informatiques» on entend tous les types d'incidents associés avec les systèmes informatiques dans lesquels les criminels reçoivent ou non des avantages financiers et qui se traduisent pour les victimes par des pertes ou des dommages directs ou indirects.

Les crimes informatiques recouvrent donc à la fois :

- les fraudes dont la motivation est d'ordre essentiellement pécunaire et qui se traduisent par une amplitude allant de quelques dizaines (un cas en Grande-Bretagne de modification d'un fichier de paye) jusqu'à plusieurs centaines de millions de francs suisse ( 5 milliards possibles à l'Equity Funding Insurance Company par revente de 56000 fausses polices d'assurances);

- les sabotages qui peuvent être vus comme un des symptômes d'un conflit culturel fondamental et de la dislocation sociale de notre société actuelle. L'amplitude des pertes peut aller de un million de francs suisses à trois millions de francs suisses : destruction du Centre Ordinateur de l'Université du Wisconsin et mort d'un analyste de système.

2. Importance du phénomène "crimes informatiques 》

a) Progression des crimes informatiques (dans le monde)

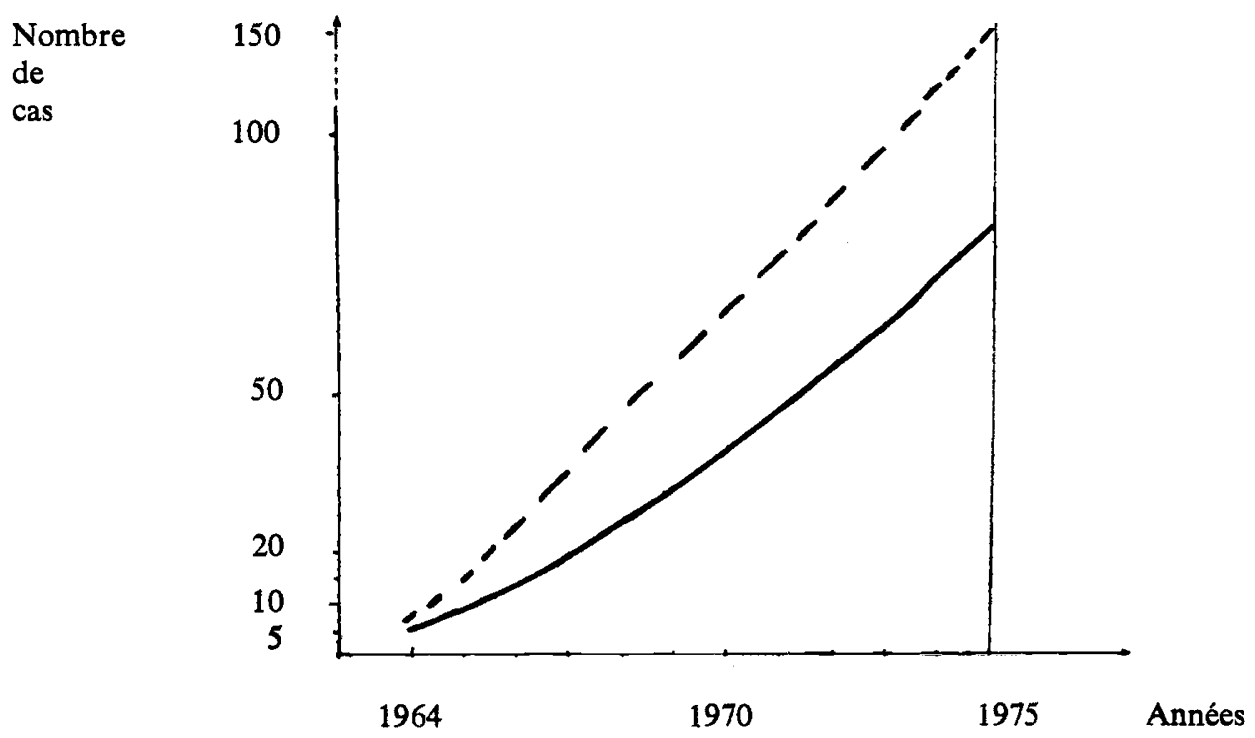

\section{Cas recensés.}

... - Estimation des cas réels (en fait les firmes répugnent fortement à avouer les crimes dont elles ont été victimes : cette estimation doit donc être considérée comme un minimum). 
b) Pertes totales en millions F.S.

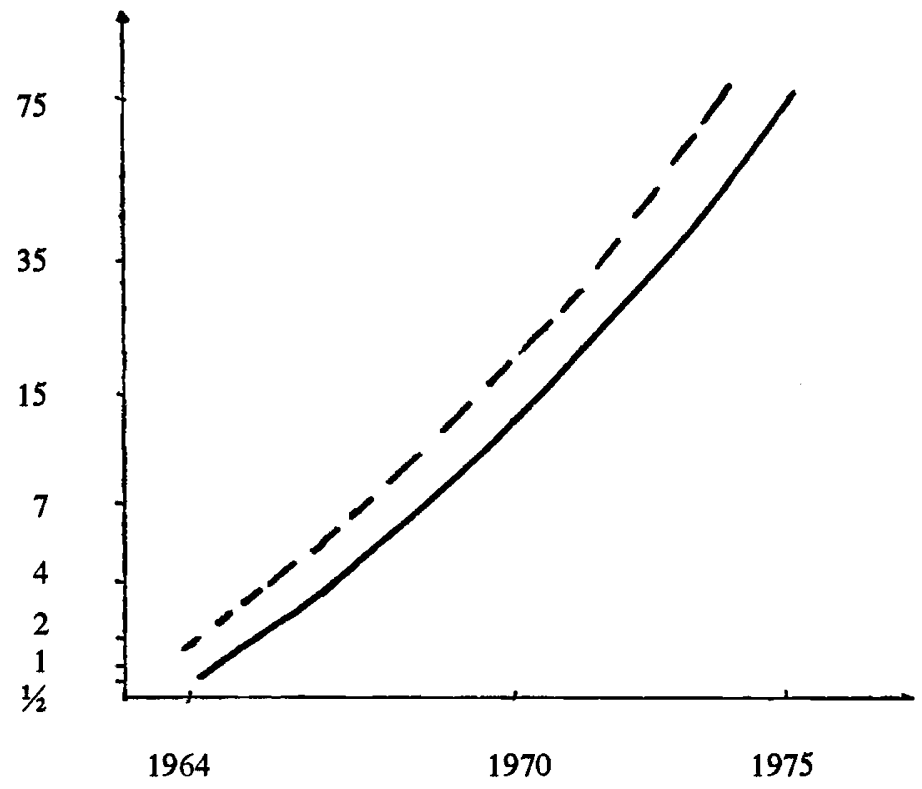

* compte non tenu d'une fraude de 5 milliards de F.S.

c) Etude des cas recensés au 1.1.75

\begin{tabular}{lrrr} 
Etats-Unis & 216 cas & soit $74 \%$ \\
Europe & 76 cas & soit $26 \%$ \\
\cline { 2 - 3 } Total & 292 cas & soit $100 \%$
\end{tabular}

Pour l'Europe occidentale: en nombre de cas.

$\begin{array}{lr}\text { RFA } & 18 \\ \text { United Kingdom } & 16 \\ \text { France } & 11 \\ \text { Scandinavie (y compris Danemark) } & 8 \\ \text { Italie } & 7 \\ \text { Divers } & 12\end{array}$


d) Analyse par secteur économique :

$\begin{array}{lcc} & \text { en nombre } & \% \\ \text { Banque et Finances } & 15 & \text { en valeur } \\ \text { Assurances } & 11 & 19 \\ \text { Commerce } & 2 & 5 \\ \text { Industrie } & 20 & 18 \\ \text { Services Publics } & 21 & 14 \\ \text { Administration } & 21 & 10 \\ \text { Services bureaux } & 10 & 15\end{array}$

Il faut remarquer que :

- les crimes informatiques au secteur Administration ont une valeur relative unitaire moins élevée que la moyenne; ceci tient au fait qu'il est actuellement difficile d'évaluer la valeur financière d'une fraude ou d'un sabotage concernant des listes électorales ou d'information sur la vie privée des citoyens (extraits, par exemple, des fichiers du Centre Ordinateur du FBI: cas cité par l'étude de la National Science Foundation);

- les crimes informatiques dans les entreprises de services (au sens général du terme), en particulier, Services Bureaux, Banques et Assurances ont une valeur relative unitaires très élevée; ceci tient particulièrement au fait que dans ces secteurs économiques la plupart des informations traitées ont:

- soit une valeur financière directe : contrepartie d'un chèque, d'un retrait d'argent, d'une police d'assurance ou d'un sinistre,

- soit une valeur financière indirecte : un des cas les plus perçants de fraude dans les services bureaux consiste à vendre les fichiers des clients à des concurrents.

\section{Essai de définition des motivations et de la typologie des criminels}

Quelques études ont essayé de rechercher et de mettre en évidence les motivations des criminels. Ces motifs ressortent principalement de deux grands domaines:

1. Les problèmes financiers.

Dans cette catégorie se placent des cas de fraude et la recherche du gain est la motivation généralement admise; quelques cas sont cependant analysés comme ayant trouvé leur source dans le challenge : homme face à la machine. 
2. A partir de cet antagonisme homme/machine, le durcissement de cette tendance conduit au "syndrome de Robin des Bois», c'est-à-dire au conflit des positions humanistes et sociales avec la rigidité "inhumaine» des systèmes informatiques.

- Devenu symbole d'une société dure et impitoyable pour l'être humain, l'informatique est attaquée comme objet de privation de liberté de choix et d'atteinte à la dignité humaine; la seconde motivation, rencontrée dans tous les cas de sabotage, est donc d'ordre essentiellement sociologique.

Cette conception se comprend très bien lorsqu'on étudie les réactions du public et souvent l'attitude de responsables de firmes face à l'informatique. Il faut remarquer notamment que :

- l'hostilité envers l'informatique, ainsi que le concept des «ordinateurs déshumanisants s'accroît (voir les réponses à des enquêtes d'opinion) :

$\begin{array}{ccc}\begin{array}{c}\text { Années } \\ \text { des enquêtes }\end{array} & \begin{array}{c}\text { Hostilité } \\ \text { envers informatique }\end{array} & \begin{array}{c}\text { Ordinateurs } \\ \text { déshumanisants }\end{array} \\ 1968 & 10 \% & 31 \% \\ 1972 & 15 \% & 54 \% \\ 1975 & 24 \% \text { des répondants. } & 62 \% \text { des répondants }\end{array}$

- dans $20 \%$ des cas de fraude, les complices (au sens légal du terme) sont des firmes, parfois très connues : elles achètent des fichiers clients, des méthodes de fabrication, du logiciel décrivant la stratégie de distribution de la concurrence...;

- dans une enquête restreinte (55 personnes) faite par le Stanford Research Institute en avril 1974, on peut trouver quelques réponses montrant à quel point le sens de l'éthique individuelle est en cours de dislocation :

- neuf clients d'un service bureau ont utilisé un programme qui ne leur appartient pas... sans bien entendu demander la permission du propriétaire légitime; deux seulement trouvent le procédé malhonnête ou illégal,

- sept personnes ont offert un programme fait pour leur employeur... à un ami ou une relation; vingt-trois trouvent ce procédé malhonnête,

- neuf personnes ont essayé de pénétrer la sécurité d'un système de time-sharing; sept trouvent ce procédé illégal ou malhonnête.

La typologie du «criminel», étudiée à partir des cas connus de fraude et de sabotage, montre que les professionnels de l'informatique sont souvent concernés (environ $85 \%$ des cas), ainsi que les utilisateurs du système informatique.

\section{Prévention et détection des fraudes et sabotages}

Il faut bien prendre conscience que la sécurité totale d'un système informatique :

- coûte très cher ;

- doit être révisée constamment pour ne pas laisser le temps aux fraudeurs et saboteurs de trouver une faille dans le système. 
En fait, on peut considérer que les remèdes, plus ou moins efficaces, selon les cas, sont d'ordre :

- technologique : emploi de dispositifs et d'équipements spéciaux qui empêchent l'utilisation de l'ordinateur à des fins criminelles;

— technique : systèmes combiné équipements - logiciel pour verrouiller les systèmes informatiques et les informations traitées ;

- légal : rien n'est encore prévu en Europe, mais quelques règlements sont en cours d'élaboration aux Etats-Unis ;

- social et humain : en remettant en cause la finalité actuelle de l'utilisation de l'informatique qui doit aussi pouvoir être un outil d'annoblissement des tâches de l'homme et un instrument de progrès social.

En réalité, dans le domaine du crime informatique, et puisque la prévention n'est pas susceptible d'apporter une sécurité suffisante à l'heure actuelle, les managers se doivent d'adopter des méthodes systématiques et quasi permanentes de détection:

- par l'emploi de la technologie et de la technique ;

- mais aussi et surtout par l'utilisation du processus d'audit des moyens informatiques de l'entreprise et de ses systèmes d'information.

La position hiérarchique est très variable; elle est en corrélation étroite avec le niveau des pertes observées: plus la position hiérarchique est élevée, plus le niveau des pertes augmente.

Deux tableaux publiés dans l'étude de la National Science Foundation (cf. Références) montrent le niveau hiérarchique des inculpés de fraude informatique pour :

- le secteur bancaire :

$\begin{array}{ll}\text { Vice Président } & 1 \\ \text { Directeur } & 4 \\ \text { Analyste } & 1 \\ \text { Chef caissier } & 1 \\ \text { Programmeur } & 3 \\ \text { Opérateur } & 2 \\ \text { Employé } & 3\end{array}$

- le secteur gouvernemental :

Directeur 1

Fonctionnaire élu 2

Divers informaticiens $\quad 16$ 


$\begin{array}{ll}\text { Employé } & 2 \\ \text { Policier } & 1 \\ \text { Divers « citoyens » } & 3\end{array}$

Menées inopinément et au moins tous les dix-huit mois pour chaque application informatisée, par des équipes mixtes internes et consultants extérieurs, ces procédures sont, de l'avis des experts, seules capables d'empêcher une fraude ou un sabotage de prendre des dimensions économiques considérables.

\section{Evolution prévisible des crimes informatiques}

Deux conceptions s'opposent :

- une hypothèse, énoncée principalement aux Etats-Unis au début des années 70, qui voudrait que l'évolution des crimes informatiques soit moins rapide que le développement de l'utilisation de l'informatique. Jusqu'à présent, les faits semblent démentir cette conception et une conférence sur ce sujet, tenue en juin 1973, n'avait pu conclure dans ce sens;

- la réaction des experts européens, consultés dans le cadre de cette étude, et qui prévoient, pour leur part, un accroissement des crimes informatiques parallèlement à l'évolution de la technologie et de la technique des systèmes informatiques.

Certains voient même dans la généralisation des crimes informatiques l'une des causes précipitant une crise sociale majeure. Pour éviter d'en arriver à ce point, l'adoption d'une déontologie informatique, la mise au point d'une législation adaptée, la prise de conscience par les managers des possibles dangers encourus, et surtout la modification profonde du rôle de l'informatique dans les entreprises et les organismes publics devraient accompagner la pénétration de plus en plus profonde de l'informatique dans notre monde.

\section{REFÉRENCES}

- Computer - Related Crime and Data Security

Stanford Research Institute - LRP - Déc. 1972

- On the Impact of the Computer on Society and Science

J. Weisenbaum

Mai 1972

- Study of International Frauds in Banks

Bank Administration Institute

1972

- White - Collar Criminal

Atherton Press, New York

- The Nature of Computer - Related Crime

Proc ACM/IEGE Conference 
- Threats to Computer Systems

Lawrence Livermore Laboratory

- Embezzlement by Computer

Proc. American Bankers Association

1973

- Computer Abuse

SRI - National Science Foundation

1973

- Data Security and Data Processing

IBM Corp.

1974

\section{Annexe D: L'assurance en informatique}

Jusqu'à présent, on peut considérer que l'assurance en informatique est largement sous-développée (certains spécialistes avancent un chiffre stupéfiant: $5 \%$ seulement des risques réels seraient couverts !).

On peut écrire que cette situation tient à un triple état de fait :

- le "risk management » n'est pas encore connu et utilisé dans de nombreuses entreprises et, à plus forte raison, dans les organismes de service public ;

- la direction générale a généralement délégué ses responsabilités en matière d'assurance à un cadre, d'origine technique dans la plupart des cas, qui ne connaît rien à l'informatique et n'est pas capable d'apprécier les pertes que ferait subir à l'entreprise un arrêt de l'exploitation;

- les responsables de l'informatique dans les entreprises évaluent bien, eux, les problèmes posés par une panne, une interruption quelconque du service, mais ne sont pas capables, par manque d'information, et de formation, de "traduire 》 ces risques ni en termes d'assurance ni en termes de "risk management».

\section{Principaux risques à garantir}

Un rapport récent d'IBM (Data Security et Data Processing, 1974) annonce deux idées qui nous semblent essentielles :

- aucun système informatique n'est impénétrable à tous les incidents possibles ;

- une organisation qui utilise des données doit accepter de les protéger.

Ces énoncés peuvent prêter à sourire par leur évidence et leur banalité, mais il faut bien considérer que, vingt ans après l'irruption de l'informatique dans l'entreprise, très peu de chefs d'entreprise, de responsables financiers et... de responsables informatiques ont réellement mené une politique franche et rationnelle dans ce domaine.

Cette étude a montré que, dans plus de $80 \%$ des cas, les panelistes étaient surpris... puis effarés... par la variété, la fréquence et l'importance des pertes possibles dans le domaine informatique. Il nous semble évident qu'il est indispensable de mener rapidement une action sérieuse d'information et de formation sur le problème du "risk management 》 (qui peut être un facteur de survie pour l'entreprise) particulièrement dans le domaine informatique. 
Quelques exemples :

— incendie au Pentagone : 6,5 millions de dollars de sinistre pour le hardware; 18 millions pour le Software;

- incendie chez Mannesmann (RFA) : 100 millions de francs de pertes.

— incendie chez Monoprix (Paris) : 50 millions de francs pour reconstituer le système... et la comptabilité Fournisseurs;

- fraude chez Equity Funding Insurance (Los Angeles) 2000 millions de dollars;

- sabotage dans un organisme gouvernemental en Angleterre: officiellement "plusieurs 》 millions de livres de dommages.

Dans tous ces cas, on peut considérer que la couverture des risques était mal faite ou incomplète.

Et plus notre société utilisera une informatique de plus en plus sophistiquée, plus les risques croîtront et pourront atteindre un niveau comparable à celui des grands incidents économiques. Il est donc nécessaire de bien identifier les risques, qui ressortent de cinq grandes catégories (pour détail, voir tableau D1).

1. Equipements

2. Données: Fichiers et Programmes

3. Perte d'exploitation

4. Responsabilité Civile

5. Divers

A l'heure actuelle, on peut penser schématiquement que :

- le risque 1 est dans la majorité des cas couvert correctement;

- le risque 2 est couvert dans une minorité de cas et généralement largement sousestimé ;

- de même que la perte d'exploitation;

- les risques 4 et 5 sont rarement assurés en Europe.

\section{L'offre de garanties}

On trouve actuellement trois types de polices d'assurances couvrant les problèmes informatiques :

- polices de type traditionnel où le risque informatique n'est pas spécialement identifié mais situé dans un contexte global qui a généralement tendance à sous-estimer les problèmes informatiques;

- polices établies spécifiquement pour un problème bien caractérisé et très défini, donc bien identifié;

- polices spéciales pour l'assurance informatique. On peut schématiser la présentation de ces polices spéciales en distinguant deux cas spécifiques :

- le modèle St Paul et Marine Insurance Co (le plus ancien, vers la fin des années 50) et qui a souvent été repris en Europe,

- le modèle CHUBB (1962). 
Une comparaison détaillée des exclusions faites par ces deux modèles est donnée au Tableau D2 joint.

Bien que, dans le domaine de l'assurance, l'informatique soit l'objet d'une concurrence active et sérieuse dans les pays européens, il nous semble souhaitable que les responsables de ces risques dans l'industrie de l'assurance puissent repenser le problème de manière globale et adaptée aux développement prévisibles à moyen terme.

\section{Tableau D1: Risques à garantir}

\section{Equipements}

- Coût de remplacement des matériels

- Coût d'aménagement des locaux et de l'environnement

- Coût des facilités techniques (conditionnement, régulation électrique, communications)

\section{Données: Fichiers et Programmes}

- Coût des supports magnétiques

- Coût de réfection des Données (hypothèse : les données existent)

- Coût de reconstitution des Données

- recherche

- mise en forme

- codification

- saisie - enregistrement

3. Perte d'exploitation

- Location des matériels

- Perte d'exploitation directe

- Perte d'exploitation indirecte (perte clientèle, perte de marchés)

4. Responsabilité Civile vis-à-vis des tiers

- Pour perte d'exploitation des tiers

- Pour dommages causés aux clients ou usagers par mauvaise ou absence d'information

- Pour dommages causés à l'environnement

5. Divers

- Coût de remise en ordre du système (travail en parallèle pendant ... mois) 


\section{Tableau D2 Computer Insurance Policy Comparison}

While there are many significant differences between the St. Paul model and the Chubb model "all-risk" computer policies, the following comparison of exclusions reveals those which are most important.

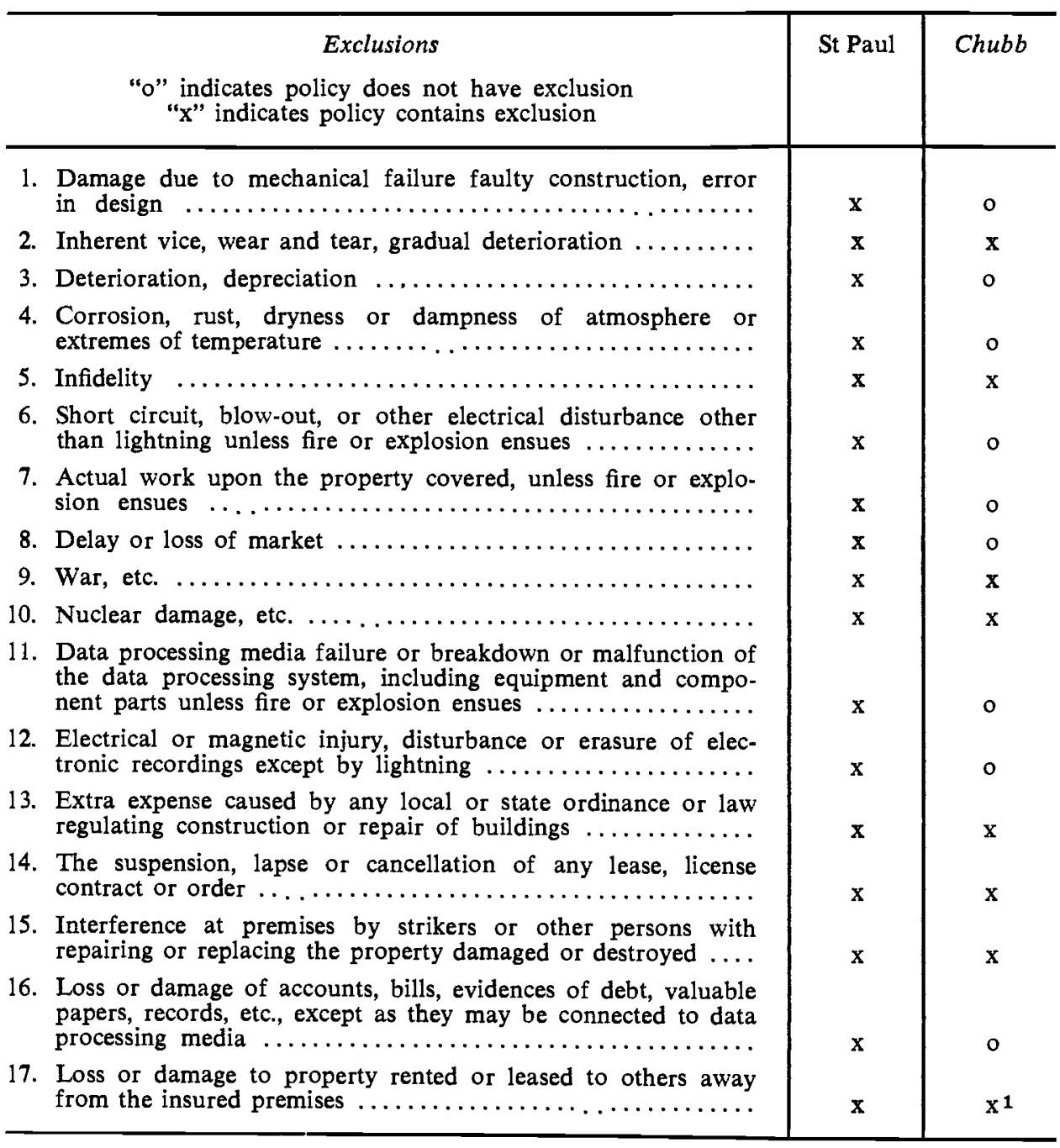

1 Not an exclusion but policy applies only to property at location(s) listed in the policy.

Tiré de «How to Insure a Computer Center», by James O. Matschulat. 


\section{QUELQUES REFFERENCES BIBLIOGRAPHIQUES}

- Data Security and Data Processing

IBM Corp.

- How to Insure a Computer Center

James O. Matschulat

1973

- Recommended Good Practice for the Protection of EDP and Industrial Automation

Factory Insurance Association

- Danger Ahead! Safeguard Your Computer

B. Allen, Harvard Business Review

Déc. 1968

- The Insurance of Computer

M. J. Harris

- Bulletin no 75, National Fire Protection Association

\section{Annexe E :}

\section{Comparalson entre l'étude de l'Association de Genéve ef l'étude SKANDIA ${ }^{1}$}

1. Remarques générales

1. Quatre années séparent la réalisation des deux études; ceci peut être une cause de distorsion importante dans un domaine de prévisions technologiques associées à des phénomènes de type socio-économique.

2. Le champ d'investigation de l'étude "Skandia 》 avait essentiellement trait aux Etats-Unis (quelques références à des systèmes sur le plan mondial) alors que l'étude "Association de Genève 》 vise l'Europe avec quelques applications sur le plan mondial.

3. Les experts réunis pour les deux panels ont été choisis en fonction de ces champs d'investigations géographiques spécifiques; d'où une attitude différente sur certains problèmes (évaluation des pertes envisageables; vitesse de pénétration de l'informatique en Europe...).

\section{Méthode de travail}

Dans les deux cas, l'étude a été basée sur l'utilisation de la méthode Delphi, mise au point par la RAND Corporation en 1964 et largement diffusée par la suite dans les problèmes de prévisions technologiques à long terme.

L'objectif de la méthode Delphi est de mettre au point un programme d'interrogations individuelles successives, réalisé avec des questionnaires alternant avec des

1 Publiée par Institute for the future, Hatford, Connecticut, USA, 1972. 
informations et les résultats issus des phases précédentes d'étude, qui permettent aux experts choisis de corriger leurs premières opinions.

En dehors des facteurs inhérents aux nationalités et à l'appartenance au groupe socio-culturel américain, la composition des panels a été sensiblement identique, avec peut-être, pour l'étude de l'Association de Genève, une plus large ouverture vers des «non-informaticiens» que dans l'étude Skandia.

\section{Résultats de l'étude}

a) Choix des applications

Toutes les applications proposées aux experts «Skandia》ont été proposées à l'exament des experts "Association de Genève 》, avec, en plus, dans ce dernier cas, une série d'applications récemment développées (traitement de textes) ou qui paraissaient prendre un essor plus rapide dans les dernières années.

Sur 20 applications retenues pour l'analyse détaillée de l'étude «Association de Genève », 9 d'entre elles figurent dans l'étude Skandia, 11 sont originales. (Donc 11 applications "Skandia 》n'ont pas été retenues : 5 d'entre elles figurent cependant dans les applications considérées par le panel "Association de Genève » comme présentant au moins $50 \%$ de chances d'être réalisées vers 1990 (cf chapitre 2,4).)

Le tableau Xy montre la comparaison détaillée sur le choix des deux panels en matière d'application.

De façon générale, les dates prévues par les experts européens pour la réalisation des applications sont beaucoup plus éloignées (six ans en moyenne) que les estimations faites par le panel Skandia sur les mêmes thèmes. *

b) Pertes globales annuelles

Les pertes globales annuelles sont, dans les deux études, d'un niveau équivalent:

$$
\begin{array}{lll}
\text { Skandia } & 2,1 & \text { milliards de dollars } \\
\text { Association de Genève } & 6,625 & \text { milliards de francs suisses }
\end{array}
$$

c) Pertes par applications

Paye et Comptabilité

Systèmes boursiers

Transactions bancaires

Industries alimentaires

Industries Pneumatiques

Raffineries

Distribution carburants

Pollution

Trafic urbain

\section{Pertes annuelles}

$\begin{array}{cc}\begin{array}{c}\text { Etude Skandia } \\ \text { (millions dollars) }\end{array} & \begin{array}{c}\text { Etude Association Genève } \\ \text { (millions F.S.) }\end{array} \\ 625 & 2.260 \\ 56 & 453 \\ 28 & 164 \\ 192 & 115 \\ 44 & 86 \\ 68 & 234 \\ 16 & 63 \\ 21 & 91 \\ 37 & 277\end{array}$

* Les six années représenteraient à la fois l'écart de réalisation des applications entre les U.S.A. et l'Europe (maximum trois années) et une estimation optimiste du premier panel (trois années également). 


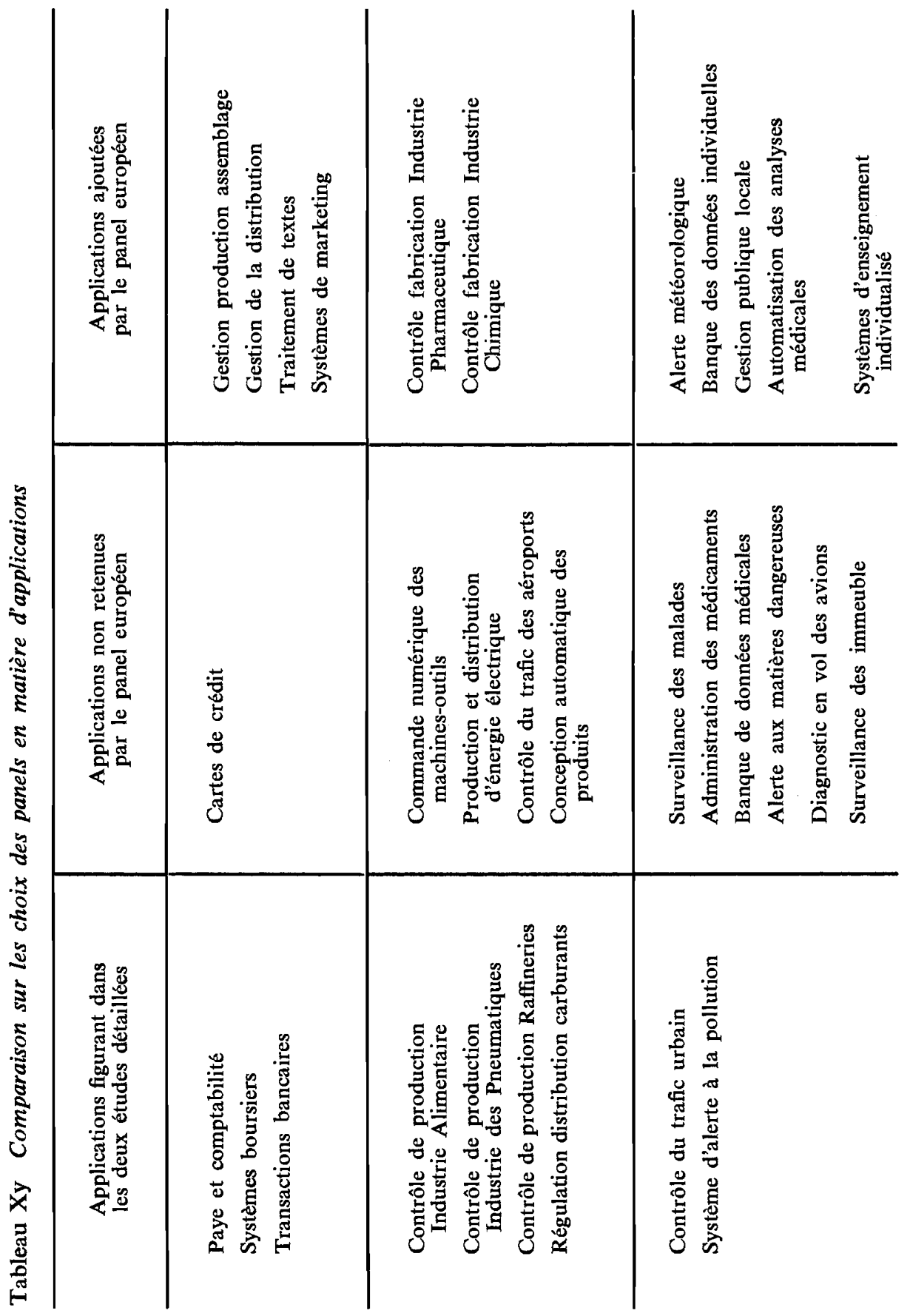


Si l'on considère les termes dans lesquels les applications ont été décrites de façon différente, par exemple, pour la paye et comptabilité :

- Skandia: $\quad 95 \%$ des firmes de plus de 500 employés...

- Association de Genève : $90 \%$ des firmes de plus de 200 employés...

on peut dire qu'il n'y a pas de véritable distorsion entre les résultats obtenus dans les deux études.

d) Fréquence et amplitude des pertes

En général, on peut écrire que le panel européen a prévu :

- une fréquence d'incidents plus importante;

- une amplitude moyenne nettement supérieure.

Skandia

Fréquence moyenne

Association Genève

800

Amplitude moyenne

e) Type de dommages

La comparaison paraît, pour ce point précis, plus difficile à établir car les catégories de ventilation des pertes par type de dommage a été faite différemment dans les deux études. De plus, une synthèse générale sur les vingt applications étudiées n'a pas été réalisée dans l'étude Skandia. Il semblerait néanmoins qu'en ce qui concerne les dommages causés aux hommes (vie, santé, réputation...) les évaluations faites par le panel «Skandia » aient été plus élevées.

f) Types d'incidents

On peut noter ici une distorsion important (en \% relatif des pertes) :

\section{Causes}

1. Equipements

2. Méthodes d'utilisation

3. Utilisateurs

4. Fraudes - Sabotages
Etude Skandia

46

16

14

12
Etude Association Genève 33

15

42

10

Le panel européen :

- craint beaucoup, en règle générale, les erreures faites par les utilisateurs soit à l'entrée des informations dans le système, soit à l'utilisation des résultats;

- affecte une valeur moindre aux incidents causés par un fonctionnement défectueux ou une panne des divers équipements (sans doute parce que la fiabilité des ordinateurs et de leurs composants s'est beaucoup améliorée depuis cinq ans).

g) Facteurs socio-économiques freins ou accélérateurs

Sauf sur quelques points spécifiques (par exemple, réduction des budgets militaires et spatiaux), l'analyse des experts du panel "Association de Genève » rejoint celle des panelistes de l'étude «Skandia ».

Les uns et les autres insistent beaucoup, en particulier, sur l'attitude sociale, en cours de développement, du rejet de l'informatique, à cause de son impact sur l'homme 
dans son travail (suppression de postes ou contraintes d'utilisation) et dans sa vie privée (fichiers de personnes, etc.).

h) Développements technologiques

En règle générale, on décèle dans l'attitude des experts européens consultés une plus grande prudence que dans les estimations faites par le panel Skandia, en particulier:

- sur la volonté des constructeurs informatiques à résoudre les problèmes par l'amélioration du software ; beaucoup d'experts pensent que l'obtention de méthodes de programmation plus performantes se fera très lentement;

- pour tout ce qui concerne les conditions économiques de l'utilisation de l'informatique; les panelistes pensent que les améliorations techniques et technologiques ne se répercuteront que faiblement sur le coût des équipements puisque, en fait, les constructeurs veulent «facturer» un service et non un matériel.

i) Evolution du marché informatique

- De manière globale, l'étude Skandia a sous-estimé * le développement de l'informatique : à fin 1975, le nombre d'ordinateurs installés dans le monde est d'au moins $50 \%$ plus élevé que les prévisions faites. Deux causes majeures :

- le bouleversement causé par l'irruption des mini-ordinateurs dans le marché : 60000 installés aujourd'hui aux Etats-Unis, soit plus que la prévision faite pour 1985 ,

- le poids spécifique du marché européen : 60000 équipements prévus pour $1985 \ldots$ alors que ce chiffre est dépassé en Europe de l'Ouest à fin 1975 (remarque valable également pour le marché japonais).

- L'étude Skandia n'a pas tenu compte* de l'abaissement du ratio cout/performance (un ordre de grandeur tous les six ans).

- La croissance globale économique de l'industrie informatique sera très nettement supérieure au taux retenu* dans l'étude Skandia.

\section{Annexe F : Les membres du panel}

Malgré leurs lourdes et constantes responsabilités, ces experts ont consacré une part importante de leur temps à répondre aux enquêtes effectuées, ne ménageant ni leur imagination ni leur sens critique. Qu'ils trouvent ici l'expression de la gratitude de l'auteur du rapport ainsi que de l'Association de Genève pour leur contribution essentielle.

Prof. Dr. C. A. Von den Beld, Director, Central Planbureau, Pays-Bas

Herr Director W. Bersin, Rheinstahl Aktiengesellschaft, Zentrale Systemsteuerung, Allemagne

Mr. Bialot, EDF - GDF, France

Mr. R. L. Bishop, Director, Treasury and Tax, Monsanto Europe, Belgique

M. A. Bonnechère, President, A.S.A.B., Belgique

Herr Dr. Bernd Braun, Anwaltsbüro, Allemagne

* Peut-être s'agit-il aussi d'un problème de définition différente des produits ? 
Mr. Massimo Brighi, Piazza Stefano Jacini 5, Italie

Univ. Prof. Dr. Gerhart Bruckmann, Ordinarius für Statistik, Autriche

Mr. M. A. Charguéraud, Président, Société Générale de Surveillance, Suisse

Mr. M. A. Chaband Latour, Banque Internationale pour l'Afrique Occidentale, France

Mr. Clerc, Président, COMSIP, France

Mr. le Prof. Pierre Denoix, Directeur, Institut Gustave-Roussy, France

Mr. W. Diehl, Deputy General Manager, Swiss Reinsurance Company, Suisse

Mr. H. Donaldson, Head of Management Services, Hill Samuel \& Co. Ltd., GrandeBretagne

Herr Dr. Eisenhofer, Leiter des Zentralbereichsorganisation und Datenverarbeitung, Brown, Boveri \& Cie Aktiengesellschaft, Allemagne

Mr. André Laleuf, Directeur Général, Comité d'Action pour la Productivité dans l'Assurance, France

Herr Ministerialdirigent Dr. A. L. Langseder, Bayerische Staatskanzlei, Abteilung Datenverarbeitung, Allemagne

Mr. Bruno Lamborghini, Direzione Studi Economici e Programmazione, Ing. C. Olivetti \& C., S.p.A., Italie

Mr. Ph. Kalevi Kontinen, Suvikummunrinne 4H24, Finlande

Mr. N. Manson, Directeur de l'Institut d'Informatique, IBM France, France

Mr. J. L. Peaucelle, IMI, c/o Centre Parisien du Management, France

Mr. A. Proeme, Philips Electorlogica B.V., Pays-Bas

Mr. Hans Raffler, Vice Director, Münchener Rückversicherungsgesellschaft, Allemagne

Mr. Don Pedro Raventus Cucurull, Seresco S.A., Espagne

Mr. L. J. Rawle, Unilever Computer Services Ltd., Grande-Bretagne

Mr. Claude Relin, France

Dottore Elio Romano, Istituto per la Ricostruzione Industriale, Italie

Mr. Etienne de Rossius, Clearly, Gottlieb Steen \& Hamilton, Belgique

Mr. Carl-Erik Schultz, Vice President, Skandia Group, Suède

Herr Prof. Dr. S. Simitis, Forschungsstelle für Juristische Dokumentation, Allemagne

Sr. Don José Antonio Suarez F. Eguiä, Director General Adjunto, Banco Espanol de Credito, Espagne

Mr. Peter Smith, Commercial Union, Grande-Bretagne

Mr. H. F. Tecoz, Le Clos d'En Coulet, Suisse

Mr. J. M. Treille, Secrétaire Général, Commission permanente de l'Electronique, Commissariat Général du Plan, France

Mr. Sven Yngvell, SAAB-SCANIA, Suède

Membres du Groupe DIEBOLD :

Henri Sherwood, Allemagne

David Buttler, Grande-Bretagne 
Summary : Research on the nature and importance of economic losses due to the utilisation of computer systems in Europe up to 1988

\section{I - AIMS OF THE STUDY - WORKING METHODS}

Aims of the study.

The main aims of the research undertaken, at the European level and with a timehorizon of 1988 , are :

- to identify the potential main applications of computers ;

- to evaluate the economic risks resulting for users in the private and public sectors;

- to refiect on possible remedial measures, particularly as regards the insurance industry.

\section{Working method}

The study took the form of three phases of investigations, some of which were carried out in parallel in several fields; the principal themes dealt with concerned three major problems :

- speed of penetration of data-processing in Europe between now and 1988;

- identification and detailed analysis of the socio-economic developments which could accelerate or slow down the growth of data-processing in the European context;

- selection of the applications likely to lead to a high level of potential economic loss; identification of foreseeable losses and estimation of the frequency and extent of such losses.

In the latter field, and on the basis of 41 applications divided into three major types of utilisation of data-processing :

- management functions ;

- control and regulation of industrial-type processes;

- applications of a public and social type,

the members of the panel selected those applications which were of interest on the basis of two main criteria :

- strong probability of functioning during the period 1975-88;

- high level of potential economic losses.

\section{II - RISKS OF POTENT1AL LOSSES}

1. In Europe the potential total losses from the 20 applications envisaged by the time-horizon of 1988 were estimated at :

\section{6,600 million Swiss francs each year}

(a general estimate relating to all automated applications could, no doubt, give a figure slightly more than double the above amount). 
2. Table 4 shows the level of losses from each of the applications studied. About $70 \%$ of these losses result from management applications ; $12 \%$ result from systems for controlling industrial processes, and $15 \%$ are imputable to applications of a public and social type.

3. The annual frequency of incidents ranges from a few units (sabotages, serious errors of programming) to 3 million (error in data acquisition), the average being about 800 . The extent of the losses per incident ranges from about 100 to 28 million Swiss francs for erroneous programming in the control of pharmaceutical production : the average figure is about 120,000 Swiss francs.

Table 6 shows in diagrammatic form the distribution of potential losses by frequency and extent.

4. The estimated losses (translated into economic terms even when the loss is of qualitative nature : loss of the clientele of an enterprise, prejudice to human health or even life...) will be distributed as follows :

$$
\begin{array}{lr}
\text { - the enterprise } & 72.5 \% \text { of the amounts } \\
\text { - the clientele } & 13.5 \% \text { of the amounts } \\
\text { - persons } & 8.5 \% \text { of the amounts } \\
\text { - miscellaneous } & 5.5 \% \text { of the amounts }
\end{array}
$$

In the field of management $95 \%$ of the losses will be direct (operating losses, loss of clientele); in applications of a public and social type 1/3 of the losses involve the human element (failure of urban traffic control systems, divulgation of confidential information, alteration of official information).

5. Table 10 gives, for all the applications studied, the distribution of annual losses by type of incident.

The experts thus considered that errors originating with the users - wrong or altered data fed into the system, bad utilisation of the results - will account for a large share $(42 \%)$ of the total losses envisaged.

Breakdowns of faulty functioning of the equipment will be responsible for $1 / 3$ of the losses. Poor working methods (erroneous programmes, incorrect utilisation of the equipment) will cause $15 \%$ of the losses; fraud and sabotage will account for $10 \%$ of the potential total losses.

Many methods were proposed to forestall incidents or reduce loss levels, the principal ones being :

- training of staff and creating an awareness of the interest of work, and improving tasks, connected with data-processing systems;

- duplication of the most vulnerable equipment or parts thereof ; rapid and sure repair procedures; maintenance designed to prevent breakdowns;

- particularly careful methods for the introduction of new systems (simulation in advance, parallel functioning of the old and the new system); 
- systematic and frequent procedures for controlling functioning : data-processing audit ;

- full insurance covering the classic contingencies (fire, damage caused by water, theft...) and, above all, operating losses and Thir-Party Liability.

\section{III - FINAL CONSIDERATIONS}

In a long-term prospective study dealing with a technological tool which is still unfamiliar and not fully understood, it is important to bear in mind that the results obtained must be taken only as general indicators capable of drawing the attention of those having top-level responsibility to possible large-scale phenomena and not of serving as a basis for finely-drawn forecasts.

At the end of this study it may be mentioned that at the present time there is, as a general rule, an almost total failure to appreciate the problem of the security of data-processing systems and the economic losses that could be envisaged. For almost all the members of the panel the initial impact of the effort of research and analysis asked of them created an awareness - comprised of both astonishment and disquiet of the extent of the risks incurred. This clearly reflects the present lack of full and exhaustive information on this problem, in particular at the level of heads of enterprises.

The principal points to which attention needs to be drawn may be summarised as follows :

- Accordingly as, on the one hand, data-processing becomes more widespread in enterprises, and, on the other hand, the data-processing systems become more complex, the risks of economic losses are going to develop considerably.

- It is possible to forestall incidents but it is too costly to be perfect in most cases ; it is thus necessary to envisage coverage of the "General Data-Processing Risk".

- The "General Data-Processing Risk" is typical of the "Management Risk" which must be conceived of as including, first, the General Management of enterprises and/or public or private bodies, the users (financiers, manufacturers, administrators), a series of specialists (data-processing experts, auditors, makers, sociologists) and... inevitably... the public authorities.

- The insurance industry is beyond doubt in a choice position to tackle the problem, to study it and to develop adequate products and strategies.

- In the absence, in good time (towards the beginning of the next decade ?...) of means of preventing, detecting and correcting data-processing incidents, it may well be considered that we will witness serious economic losses which will exacerbate still more the phenomenon of rejection of a computerised society and will thus pose a real threat of breakdown of the socio-economic mechanisms of our world.

This research effort concludes unambiguously that it is now necessary to reflect in depth on all aspects of the dangers represented by the growth of data-processing in our society and to define what should come under the scope of preventive action and what the "General Data-Processing Risk" should cover. 
Zusammenfassung : Studie über dle Art und Bedeutung wirtschaftlicher Verluste bel Anwendung der Datenverarbeitunssysteme In Europa bls 1988

\section{I - ZIELSETZUNG - ARBEITSMETHODEN \\ Zweck der Studie}

Die durchgeführten Forschungsarbeiten hatten vor allem den Zweck, auf europäischer Ebene und bis zum Jahre 1988 :

- die wichtigsten potentiellen Anwendugsgebiete für Computer zu bestimmen;

- die wirtschaftlichen Risiken abzuschätzen, die mit der Verwendung von Computern auf dem privaten und öffentlichen Sektor entstehen;

- sich, vor allem auf Versicherungsebene, Gedanken über die möglichen Massnahmen für die Risikoverminderung zu machen.

\section{Arbeitsmethoden}

Die Studie wurde in drei Befragungsphasen durchgeführt, wobei bestimmte Befragungen auf mehreren Sektoren parallel verliefen. Die wichtigsten der behandelten Themen betreffen drei Hauptprobleme :

- ein Urteil darüber, wie schnell die Datenverarbeitung bis 1988 in Europa vordringen wird ;

- Bestimmung und detaillierte Analyse der sozio-ökonomischen Vorgänge, die die Entwicklung der Datenverarbeitung auf europäischer Ebene beschleunigen bzw. bremsen können ;

- eine Auswahl von Anwendungen, die möglicherweise zu einem hohen Niveau potentieller wirtschaftlicher Schäden führen; Bestimmung der möglichen Verluste und Abschätzen von Frequenz und Amplitude der Verluste.

$\mathrm{Zu}$ diesem letzten Punkt und ausgehend von 41 Anwendungen, aufgefächert nach den drei wichtigsten Typen der EDV-Anwendung:

- Unternehmensführung ;

- Prozess-Steuerung und Regelvorgänge bei der industriellen Fertigung ;

- Anwendungen auf dem öffentlichen und privaten Sektor,

haben die Mitglieder der Studiengruppe die interessantesten Anwendungen den zwei wichtigsten Kriterien entsprechend ausgewählt, nämlich :

— nach hoher Anwendungswahrscheinlichkeit im Zeitraum 1975 - 1988;

- nach hohem Niveau potentieller wirtschaftlicher Verluste.

\section{II - DAS RISIKO POTENTIELLER VERLUSTE}

1. Für Europa wurden die potentiellen Gesamtverluste für 20 bis 1988 vorgesehene Anwendungen auf

\section{6,6 Milliarden Schweizer Franken jährlich}

geschätzt (eine allgemeine Schätzung über die Gesamtheit der automatisierten Anwendungen könnte ohne Zweifel etwas mehr als den doppelten Wert erreichen).

2. Die Tafel 4 zeigt das Niveau des Verluste für jede der untersuchten Anwendungen. Etwa $70 \%$ der Verluste sind das Ergebnis von Anwendungen in der Unternehmens- 
führung : $12 \%$ stammen aus den Systemen für die Prozess-Steuerung und $15 \%$ gehen auf Anwendungen im öffentlichen und privaten Bereich zurück.

3. Die jährliche Frequenz der Störungen variiert von einigen Einheiten (Sabotage, schwerwiegende Programmierfehler) bis 3 Millionen (Fehler in der Erfassung von Information) - mit einem Mittelwert von etwa 800.

Die Amplitude der durch Betriebsstörungen bedingten Verluste reicht von rund Einhundert bis 28 Millionen Schweizer Franken für falsche Programme bei der Steuerung von Fabrikationsprozessen in der pharmazeutischen Industrie : Der Mittelwert liegt bei etwa 120 000.- Schweizer Franken.

Die Tafel 6 zeigt schematisch die Auffächerung der potentiellen Verluste nach Frequenz und Amplitude.

4. Die geschätzten Schäden (auf den wirtschaftlichen Bereich bezogen - auch dann, wenn es sich um qualitative Schäden handelt : Kundenverluste eines Unternehmens, gesundheitliche Schäden und Todesfälle...) betreffen :

$$
\begin{array}{llrl}
\text { - Unternehmen } & \mathrm{zu} & \mathbf{7 2 , 5} \% \text { der Gesamtsumme } \\
\text { - Kunden } & \mathrm{zu} & \mathbf{1 3 , 5} \% \text { der Gesamtsumme } \\
\text { - Menschen } & \mathrm{zu} & 8,5 \% \text { der Gesamtsumme } \\
\text { - Verschiedenes } & \mathrm{zu} & 5,5 \% \text { der Gesamtsumme }
\end{array}
$$

Bei den Verlusten auf dem Gebiet der Unternehmensführung handelt es sich zu $95 \%$ um direkte Schäden (Umsatzverluste, Kundenverluste). Bei den Anwendungen auf dem öffentlichen und sozialen Sektor betrifft 1/3 der Verluste den Menschen (Mängel in den Systemen für die Regelung des Stadtverkehrs, Veröffentlichung von vertraulichen Information, Verfälschung von Bekanntmachungen).

5. Die Tafel 10 zeigt - für sämtliche untersuchten Anwendungen — die Auffächerung der jährlichen Verluste nach Art der Störung.

Die Experten sind daher davon ausgegangen, dass die durch das Personal bedingten Fehler : Eingabe von falschen oder verfälschten Daten in das System, falsche Auswertung der Ergebnisse etc., einen Grossteil (42\%) der in Betracht gezogenen Verluste ausmachen.

1/3 der Verluste entfällt auf Pannen oder mangelhaften Betrieb der Anlagen. Ungeeignete Arbeitsmethoden (fehlerhafte Programme, falscher Betrieb der Anlage) sind die Ursache für $15 \%$, Betrug und Sabotage für $10 \%$ der potentiellen Gesamtverluste.

Es wurden mehrere Verfahren vorgeschlagen, die geeignet sind, Störungen vorherzusagen bzw. das Niveau der Verluste zu senken. Hierzu zählen :

- die Ausbildung des Personals, Stimulierung des Interesses an der Arbeit und an der Beschäftigung im EDV-Bereich;

- Verdoppelung der Einrichtungen oder der empfindlichsten Teile. Wirksame und schnelle Behebung von Störungen, vorbeugender Unterhalt der Anlage;

- besonders sorgfältig vorbereitete Methoden für die Einführung von neuen Systemen (vorherige Simulierung, Parallelbetrieb der vorhandenen und der neuen Anlage);

- systematische und häufige Funktionsprüfungen : EDV-Prüfung ; 
- ein vollständiges Versicherungssystem, das die herkömmlichen Schadensfälle (Feuer, Wassereinbruch, Diebstahl) und vor allem Betriebsunfälle und die Haftpflicht deckt.

\section{III - SCHLUSSBETRACHTUNGEN}

Bei der Durchführung einer prospektiven Studie langfristigen Charakters über die Entwicklung eines noch wenig bekannten und schlecht beherrschten technologischen Werkzeugs ist vor allem die Feststellung wichtig, dass die erzielten Resultate lediglich als allgemeine Anhaltspunkte gewertet werden können, die zwar geeignet sind, höchste Stellen für mögliche Phänomene mit starken Auswirkungen $\mathrm{zu}$ interessieren, die jedoch nicht als Basis für detaillierte Prognosen verwendet werden können.

Wie die Untersuchungen ergeben haben, wird das Problem der Sicherheit von Datenverarbeitungsanlagen und der potentiellen wirtschaftlichen Verluste im allgemeinen fast gänzlich verkannt. Für praktisch sämtliche Mitarbeiter der Befragungsgruppe hat sich der geforderte Forschungs- und Analysenaufwand zunächst darin ausgedrückt, dass man sich angesichts des Ausmasses der Risiken mit Erstaunen und mit Beunruhigung des Problems bewusst geworden ist. Insbesondere auf der Direktionsebene spiegelt sich sehr gut wider, dass umfassende und vollständige Informationen zu diesem Problem völlig fehlen.

Die wichtigsten Punkte, die herauszustellen sind, lassen sich schematisch wie folgt beschreiben :

- Mit dem verstärkten Eindringen der Datenverarbeitung in die Unternehmen einerseits und der wachsenden Komplexität der EDV-Einrichtungen andererseits werden sich die Risiken wirtschaftlicher Verluste mit der Zeit beträchtlich erhöhen.

- Vorbeugende Massnahmen gegen Störungen sind möglich, jedoch in den meisten Fällen zu teuer, um vollkommen zu sein. Es ist daher unumgänglich, eine Deckung des "Allgemeinen EDV-Risikos" vorzusehen.

- Das " Allgemeine EDV-Risiko" - von der Art des " Management-Risikos" - muss wie ein umfassendes System aussehen, das zunächst die Direktion der Unternehmen und/oder die Behörden und private Stellen, die Anwender (Finanzwelt, Produzenten, Verwaltung) und eine Reihe von Spezialisten (EDV-Fachleute, Prüfer, Konstrukteure, Soziologen usw.) und... unvermeidlicherweise auch die Öffentliche Hand einschliesst.

- Die Versicherungsbranche hat ohne Zweifel die Wahl, das Problem nach Belieben zu erfassen, zu untersuchen und geeignete Produkte und Strategien zu entwickeln.

- Da zur gewünschten Zeit (gegen Beginn der 80er Jahre?...) keine vorbeugenden Massnahmen oder Mittel für die Bestimmung und Behebung von Störungen im EDV. Betrieb zur Verfügung stehen werden, kann man zukünftig mit schweren wirtschaftlichen Schäden rechnen, die das Phänomen der Ablehnung durch die computerisierte Gesellschaft noch verstärken, die somit die reelle Gefahr mit sich bringen, dass die sozio-ökonomischen Mechanismen unserer Welt blockiert werden.

Diese Forschungsarbeiten führen klar zu dem Schluss, dass es bereits heute notwendig ist, ganz allgemein die durch die verstärkte Computerisierung unserer Gesellschaft bedingten Gefahren $\mathrm{zu}$ bedenken und zu bestimmen, was sich mit vorbeugenden Massnahmen erreichen lässt und was das "Allgemeine EDV-Risiko" decken soll. 